\title{
2008
}

Oak Ridge National Laboratory

Annual IIIness and Injury

Surveillance Report

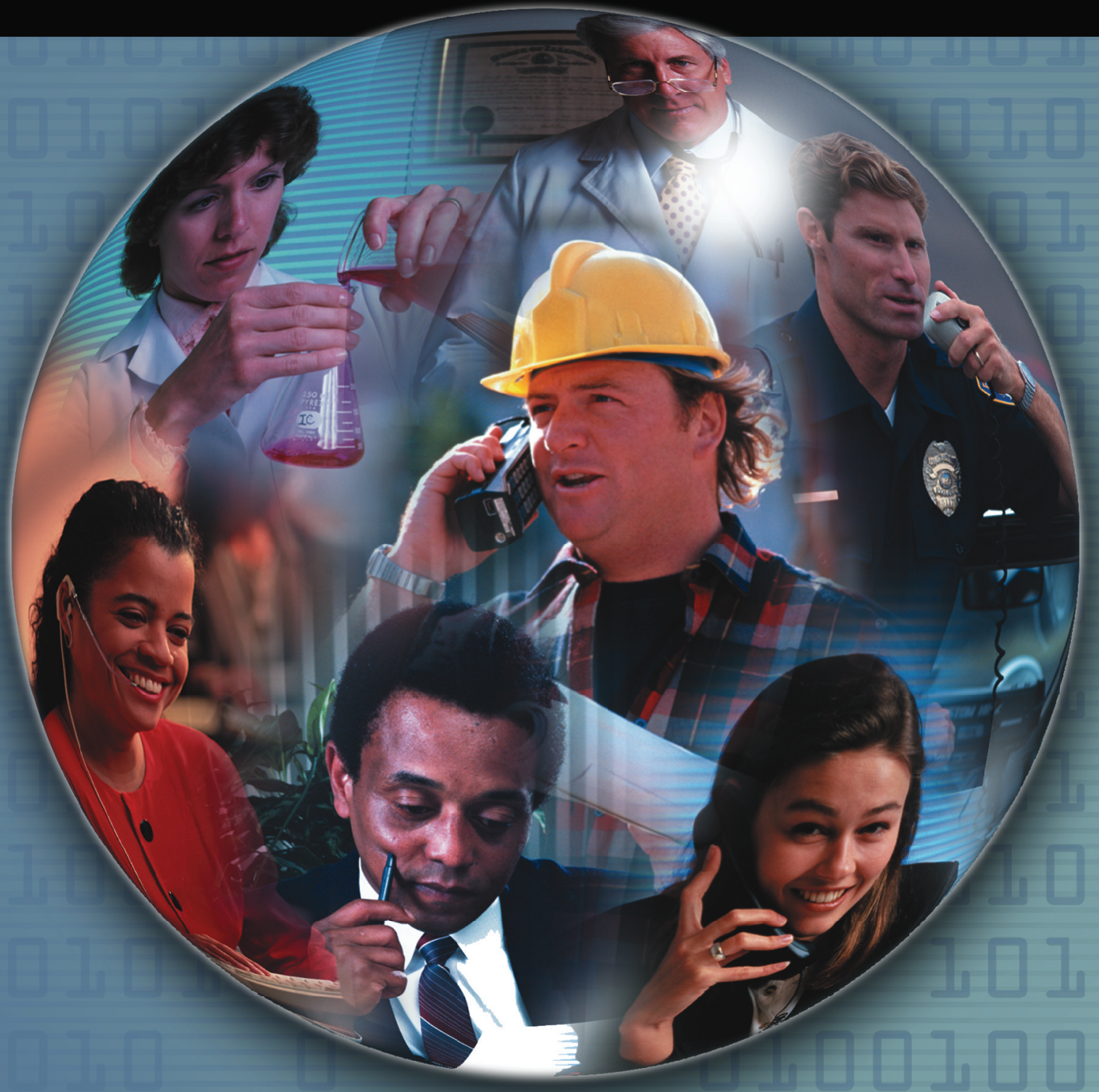




\section{Oak Ridge National Laboratory 2008 Illness and Injury Surveillance Report}

Questions or comments about this report or the Illness and Injury Surveillance Program (IISP) may be directed to:

E-mail:

Dr. Cliff Strader at cliff.strader@hq.doe.gov or Dr. Bonnie Richter at bonnie.richter@hq.doe.gov

or direct letters to:

Mail Stop HS-13 / 270CC

U.S. Department of Energy

1000 Independence Avenue, S.W.

Washington, DC 20585-0270

Additional information about the Department of Energy's Office of Illness and Injury Prevention Programs, the IISP, and annual reports for DOE sites participating in this program can be found at:

http://www.hss.energy.gov/healthsafety/WSHP/epi/surv/

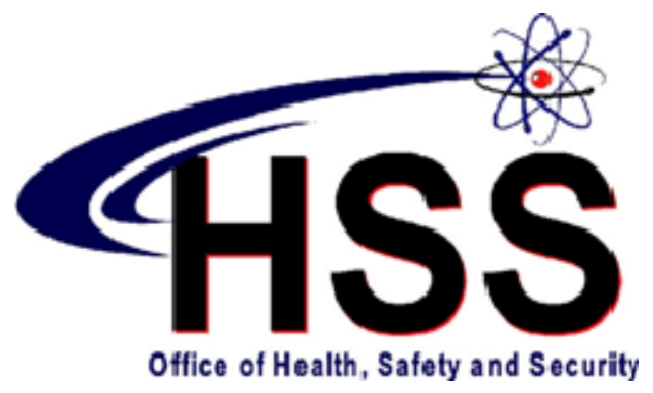

ACKNOWLEDGEMENT

LifeART images copyright 2000 Lippincott Williams \& Wilkins. All rights reserved.

This document was produced under contract number DE-AC05-06OR23100 between the

U.S. Department of Energy and Oak Ridge Associated Universities. 


\section{Y-12 National Security Complex \\ 2008 Illness and Injury Surveillance Report \\ At A Glance}

The Y-12 work force consisted of 5,027 employees in 2008. The size of the work force has been about 5,000 since 2003. Women made up about 27 percent of all workers, which is consistent with other DOE sites participating in the Illness and Injury Surveillance Program (IISP).

There were 978 absences reported in 2008, an increase of 73 absences from 2007 but less than the high of 1,043 absences reported in 2003.

Line Operators had the highest absence rate among men and Service workers had the highest absence rate among women in 2008. Professional workers had the lowest absence rate for men and Crafts workers had the lowest absence rate for women. There was little change in absence rates from 2003 to 2008 among men in the Line Operators and Professional job categories. Among women, the absence rates for Service workers declined from 2003 to 2005 and then increased from 2006 to 2008 , with the 2008 rate returning to the same level as in 2003. Absence rates for women in the Crafts group declined dramatically, with a greater than five-fold decrease in rates during the period 2003 to 2008 .

Among both men and women, disorders of the musculoskeletal system and respiratory conditions have been among the 3 more common diagnoses since 2000. These 2 diagnostic categories are typically among the 3 more commonly reported at DOE sites participating in the IISP.

Women lost 12,229 calendar days due to injury and illness with an average length of absence of 35 days. Men lost 25,560 calendar days due to injury and illness with an average absence lasting 41 days. The average length of absences has remained stable since 2003 for both men and women.

Age-adjusted rates for all illness and injury diagnoses combined have been fairly constant for both men and women since 2003. 
Eighteen diagnoses for sentinel health events were reported in 2008. Eight diagnoses involved carpal tunnel syndrome, with 3 of the diagnoses reported by female Professional workers and 3 diagnoses reported by male Line Operators. There were 4 respiratory system diagnoses, 2 of which were for berylliosis reported by 2 male workers (1 Crafts worker and 1 Service worker). The remaining diagnoses were reported for 3 other organ systems.

There were 15 OSHA-recordable events among women and 52 events among men. The rate of OSHA events among the Y-12 work force in 2008 was 1 per 100 workers, a decrease from 2007. OSHA event rates have declined at Y-12 since 2003, similar to the decline that has been observed nationally and within DOE.

Injuries accounted for 69 percent of the diagnoses reported by men and 48 percent of the diagnoses among women. The most common types of injuries were open wounds and sprains and strains among men and sprains and strains and fractures among women. Two employees ( 1 female Service worker and 1 male Crafts worker) reported exposure to an airborne irritant.

Among women, Crafts workers had the highest rate of OSHA events (4 per 100 workers); Line Operators had the highest rate of OSHA events among men (5 per 100 workers). Administrative Support workers had the lowest rate of OSHA events among women. Male Technical Support workers reported no OSHA events in 2008.

The number of OSHA events reported by men decreased less than 5 percent from 56 events in 2007 to 52 events in 2008. However, the total number of lost and restricted days declined dramatically for men, from 1,192 days in 2007 to 527 days in 2008 (56 percent decline). We observed a similar decline in the average number of lost and restricted workdays in 2008 (10 days versus 21 days in 2007). Female workers also reported decreases in number of OSHA events and workdays lost and restricted in 2008, but they were smaller than the decreases for men. 


\section{The Oak Ridge National Laboratory Work Force - 2008}

The Work Force by Gender and Age ............ 1

The Work Force by Gender and Job

Category 1

Number and Length of Absences

Absence Rate by Gender and Age 2

Number of Days Absent by

Gender and Age

Absence Rate by Job Category

and Gender 3

Average Duration of Absence by

Job Category and Gender. .. 3

\section{Diagnostic Categories}

Number of Diagnoses and Lost Calendar Days by Diagnostic Category

(Categorized by ICD-9-CM) and Gender . .4

Common Diagnoses Among Female

Workers in 2008 .. 5

Common Diagnoses Among Male

Workers in 2008 .. 6

Number of Most Frequently Reported Diagnoses by Job Category and Gender 7

\section{Rates of Disease Occurrence}

Rates for All Illnesses and Injuries Combined by Job Category, Gender, and Age

Rates for Selected Diagnostic Categories by Job Category, Gender, and Age .. 8

\section{Time Trends}

Age-Adjusted Rates for All Diagnoses Combined Among Women and Men from 2003 to 2008
Age-Adjusted Rates for Selected Diagnostic Categories Among Women and Men from 2003 to 2008

Age-Adjusted Rates for All Diagnoses

Combined Among Women and Men by Job

Category from 2003 to $2008 \ldots \ldots \ldots \ldots \ldots \ldots \ldots \ldots . \ldots 12$

\section{Sentinel Health Events for Occupations (SHEOs)}

Characteristics of SHEOs by Gender 13

SHEO Diagnoses by Gender 13

Occupational Safety and Health Administration (OSHA)-Recordable Events

OSHA-Recordable Events by Gender and Age. 14

OSHA-Recordable Events by Job

Category and Gender 14

\section{Diagnostic and Accident Categories for OSHA-Recordable Events}

OSHA-Recordable Diagnoses by

Diagnostic Category and Gender

OSHA-Recordable Accidents by Type

and Gender

Rates of OSHA-Recordable Events

OSHA-Recordable Rates by Age and Job

Categories Among Women, All Diagnoses

Combined

OSHA-Recordable Rates by Age and Job

Categories Among Men, All Diagnoses

Combined

Time Trends for OSHA-Recordable Events

Age-Adjusted Rates for All OSHA-Recordable Diagnoses Combined Among Women and Men by Job Category from 2003 to 2008 .

\section{Appendices}

Appendices A-W .20 
The Oak Ridge National Laboratory Work Force - 2008

Figure 1. The Work Force by Gender and Age

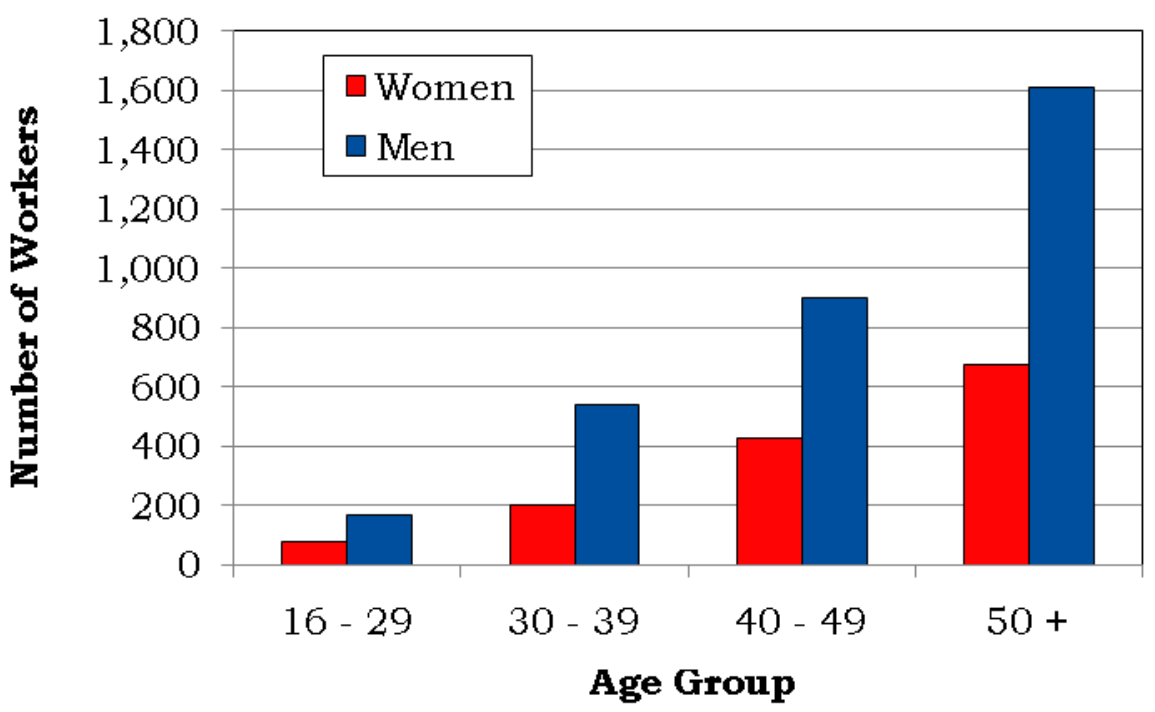

Figure 2. The Work Force by Gender and Job Category

\begin{tabular}{|l|c|c|}
\hline \multicolumn{1}{|c|}{ Job Category } & W omen & Men \\
\hline Professional & 692 & 2,275 \\
& $50 \%$ & $\mathbf{7 1 \%}$ \\
\hline \multirow{2}{*}{ Administrative Support } & 478 & 23 \\
& $34 \%$ & $1 \%$ \\
\hline \multirow{2}{*}{ Technical Support } & 116 & 294 \\
& $\mathbf{8 \%}$ & $\mathbf{9 \%}$ \\
\hline \multirow{2}{*}{ Service } & 66 & 142 \\
& $5 \%$ & $4 \%$ \\
\hline \multirow{2}{*}{ Security and Fire } & 13 & 47 \\
& $1 \%$ & $1 \%$ \\
\hline \multirow{2}{*}{ Crafts } & 15 & $\mathbf{3 8 8}$ \\
& $1 \%$ & $12 \%$ \\
\hline \multirow{2}{*}{ Line Operators } & 1 & $\mathbf{5 4}$ \\
& $<1 \%$ & $2 \%$ \\
\hline \multirow{2}{*}{ Total } & 1,381 & $\mathbf{3 , 2 2 3}$ \\
& $100 \%$ & $100 \%$ \\
\hline
\end{tabular}




\section{Number and Length of Absences}

Figure 3. Absence Rate by Gender and Age

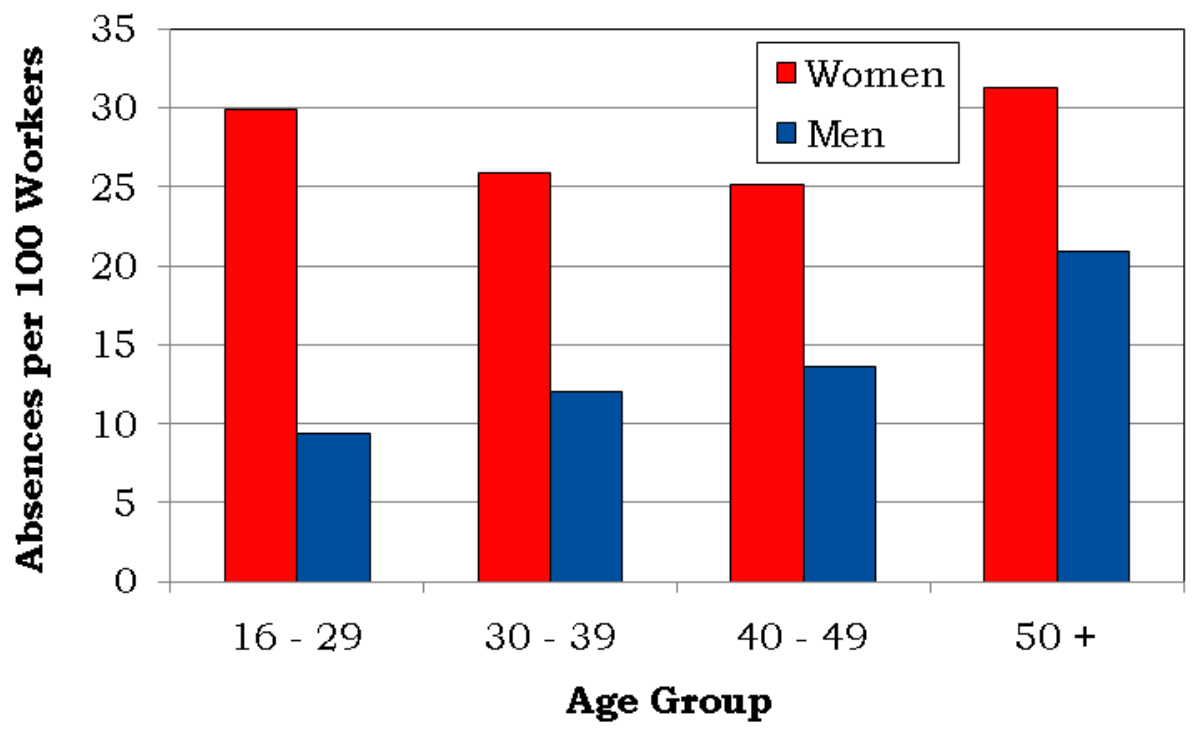

Figure 4. Number of Days Absent by Gender and Age

\begin{tabular}{|c|c|c|c|c|}
\hline \multirow{3}{*}{ Gender } & \multirow{2}{*}{ Age } & \multirow{2}{*}{$\begin{array}{c}\text { Number of } \\
\text { Absences }\end{array}$} & \multicolumn{2}{|c|}{ Number of Days Absent } \\
\cline { 3 - 5 } & & 23 & Total & Average \\
\hline \multirow{4}{*}{ Women } & $16-29$ & 52 & 126 & 5 \\
\cline { 2 - 5 } & $30-39$ & 108 & 2,317 & 17 \\
\cline { 2 - 5 } & $40-49$ & 211 & 4,942 & 21 \\
\cline { 2 - 5 } & $50+$ & 394 & 8,250 & 23 \\
\cline { 2 - 5 } & Total & 16 & 143 & 21 \\
\hline \multirow{4}{*}{ Men } & $16-29$ & 65 & 1,011 & 16 \\
\cline { 2 - 5 } & $30-39$ & 123 & 2,608 & 21 \\
\cline { 2 - 5 } & $40-49$ & 337 & 8,787 & 26 \\
\cline { 2 - 5 } & $50+$ & 541 & 12,549 & 23 \\
\cline { 2 - 5 } & Total & & & \\
\cline { 2 - 5 } & & & & \\
\hline
\end{tabular}


Figure 5. Absence Rate by Job Category and Gender

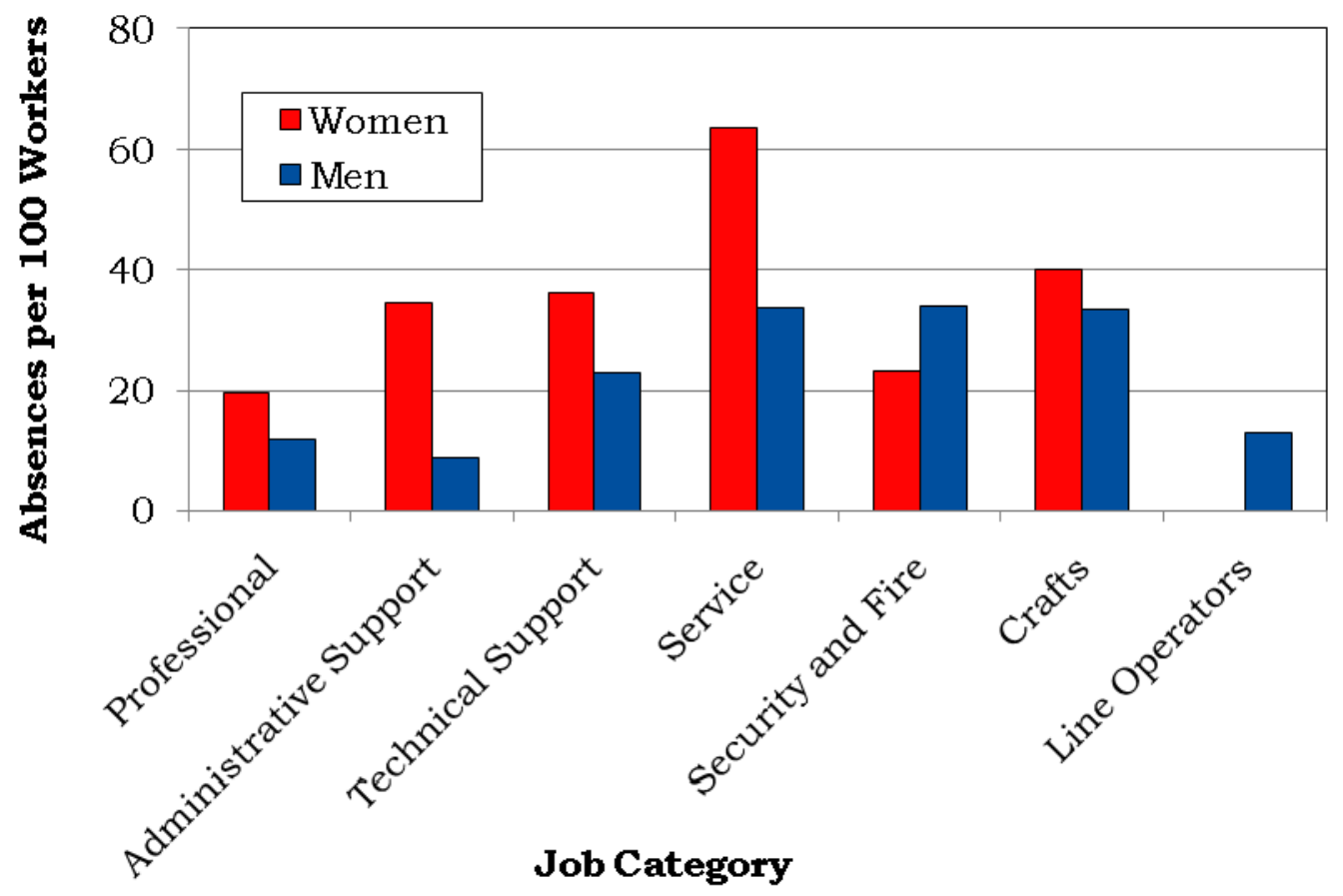

Figure 6. Average Duration of Absence by Job Category and Gender

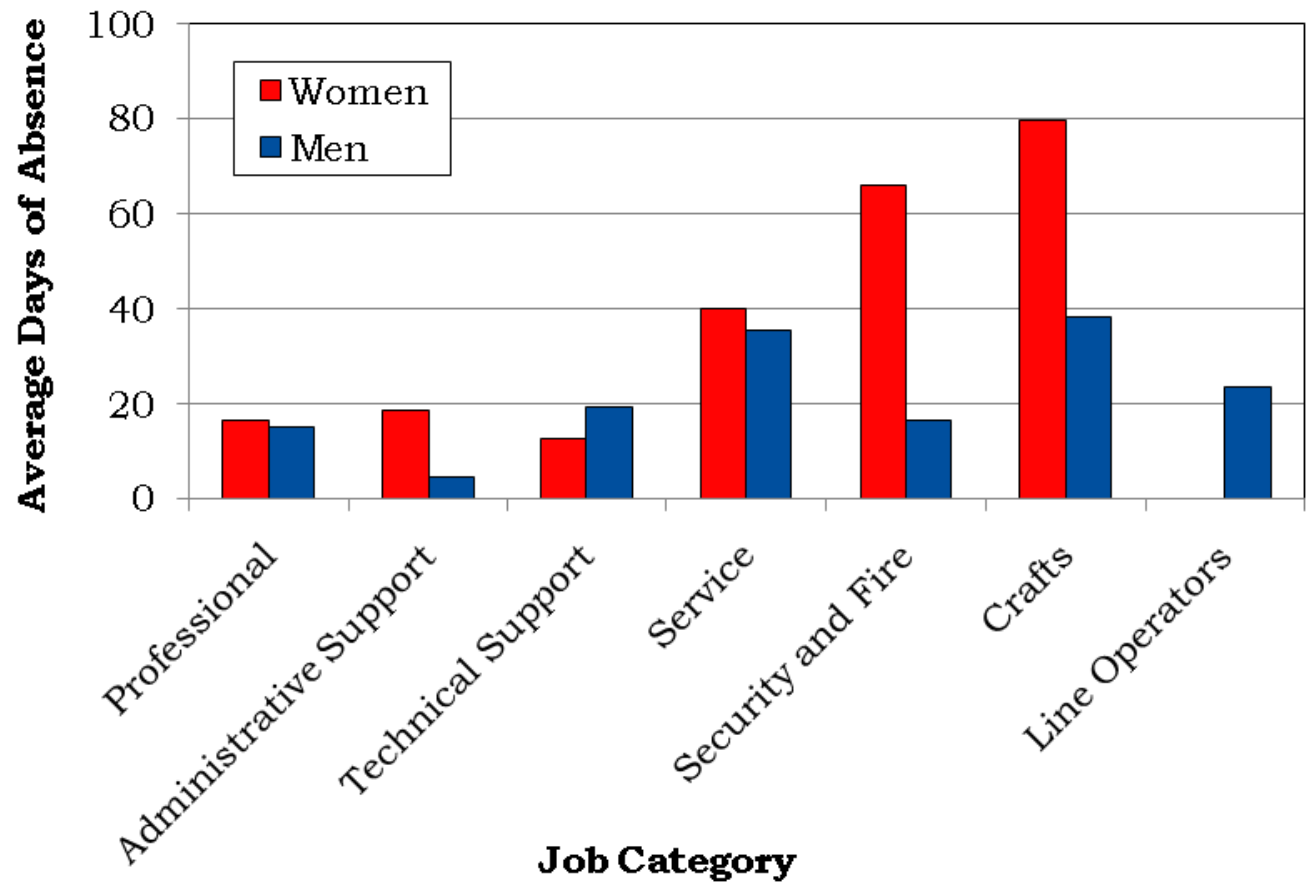




\section{Diagnostic Categories}

Figure 7. Number of Diagnoses and Lost Calendar Days by Diagnostic Category (Categorized by ICD-9-CM) and Gender

\begin{tabular}{|c|c|c|c|c|}
\hline \multirow[b]{2}{*}{ Diagnostic Category } & \multicolumn{2}{|c|}{ Women } & \multicolumn{2}{|c|}{ Men } \\
\hline & $\begin{array}{l}\text { Number of } \\
\text { Diagnoses }\end{array}$ & $\begin{array}{c}\text { Number } \\
\text { of Lost } \\
\text { Calendar } \\
\text { Days }\end{array}$ & $\begin{array}{l}\text { Number of } \\
\text { Diagnoses }\end{array}$ & $\begin{array}{c}\text { Number } \\
\text { of Lost } \\
\text { Calendar } \\
\text { Days }\end{array}$ \\
\hline Benign Growths & 16 & 441 & 10 & 225 \\
\hline Blood & 2 & 84 & 0 & 0 \\
\hline Cancer & 20 & 894 & 15 & 421 \\
\hline Digestive & 57 & 719 & 88 & 1,001 \\
\hline Endocrine / Me tabolic & 11 & 463 & 4 & 157 \\
\hline Existing Birth Condition & 2 & 77 & 1 & 2 \\
\hline Genitourinary & 38 & 521 & 27 & 212 \\
\hline Heart/Circulatory & 19 & 293 & 45 & 956 \\
\hline Infections/Parasites & 28 & 390 & 34 & 471 \\
\hline Injury & 61 & 1,716 & 130 & 3,902 \\
\hline Miscarriage & 3 & 40 & NA & NA \\
\hline Musculoskeletal & 80 & 2,309 & 121 & 4,349 \\
\hline Nervous System & 40 & 773 & 36 & 607 \\
\hline Psychological & 11 & 479 & 11 & 690 \\
\hline Respiratory & 116 & 879 & 126 & 1,188 \\
\hline Skin & 6 & 41 & 10 & 277 \\
\hline Unspecified Symptoms & 38 & 677 & 48 & 561 \\
\hline
\end{tabular}

Note: Lost calendar days for each absence are counted more than once when multiple diagnoses occur in different diagnostic categories for the same absence. 


\section{Figure 8. Common Diagnoses Among Female Workers in 2008}

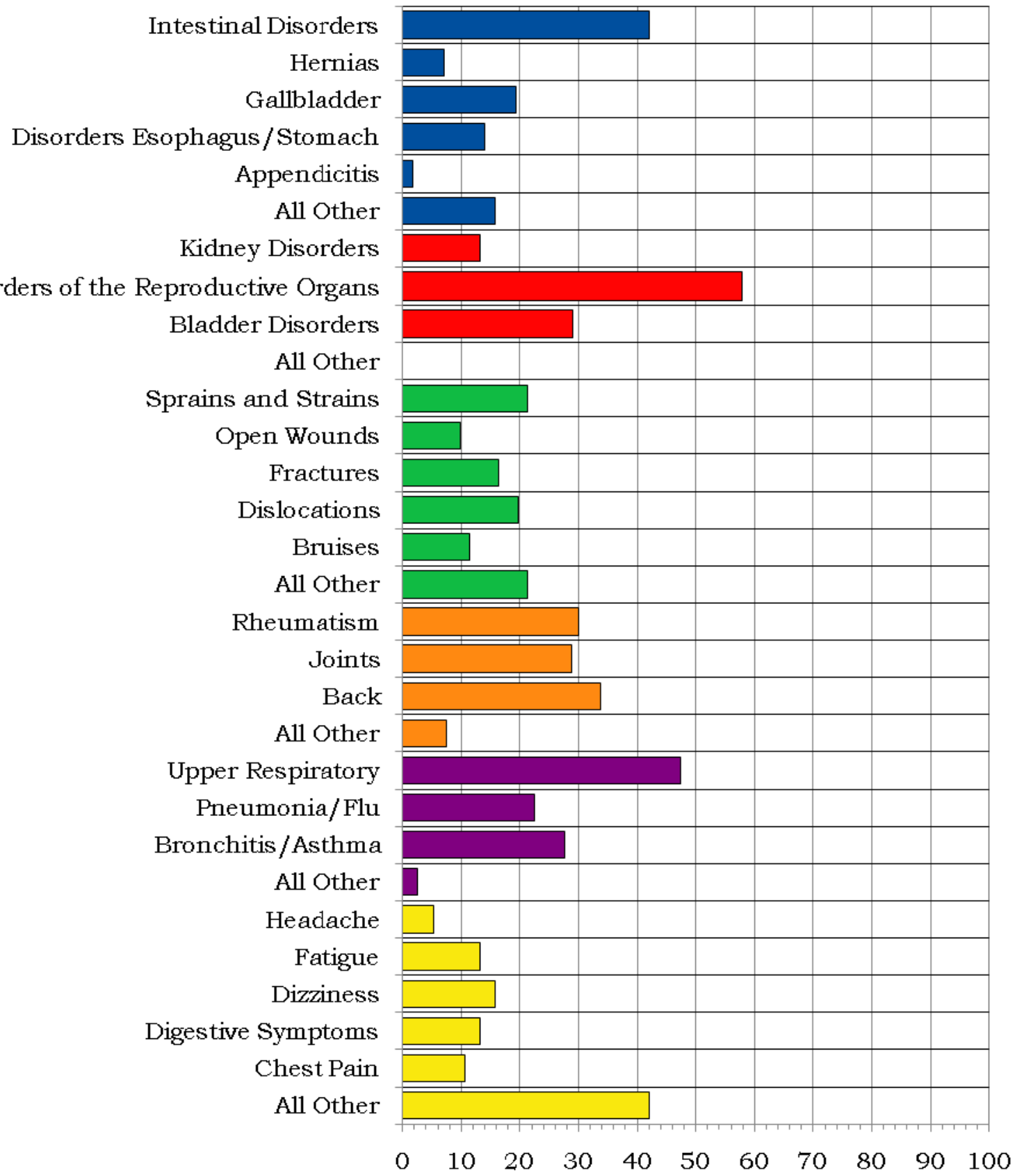

Percent Distribution of Diagnoses Within Diagnostic Category

Dige stive, 57 Diagnoses

Genitourinary, 38 Diagnoses

Injury, 61 Diagnoses
Musculoskeletal, 80 Diagnoses

Respiratory, 116 Diagnoses

Unspecified Symptoms, 38 Diagnoses 
Figure 9. Common Diagnoses Among Male Workers in 2008

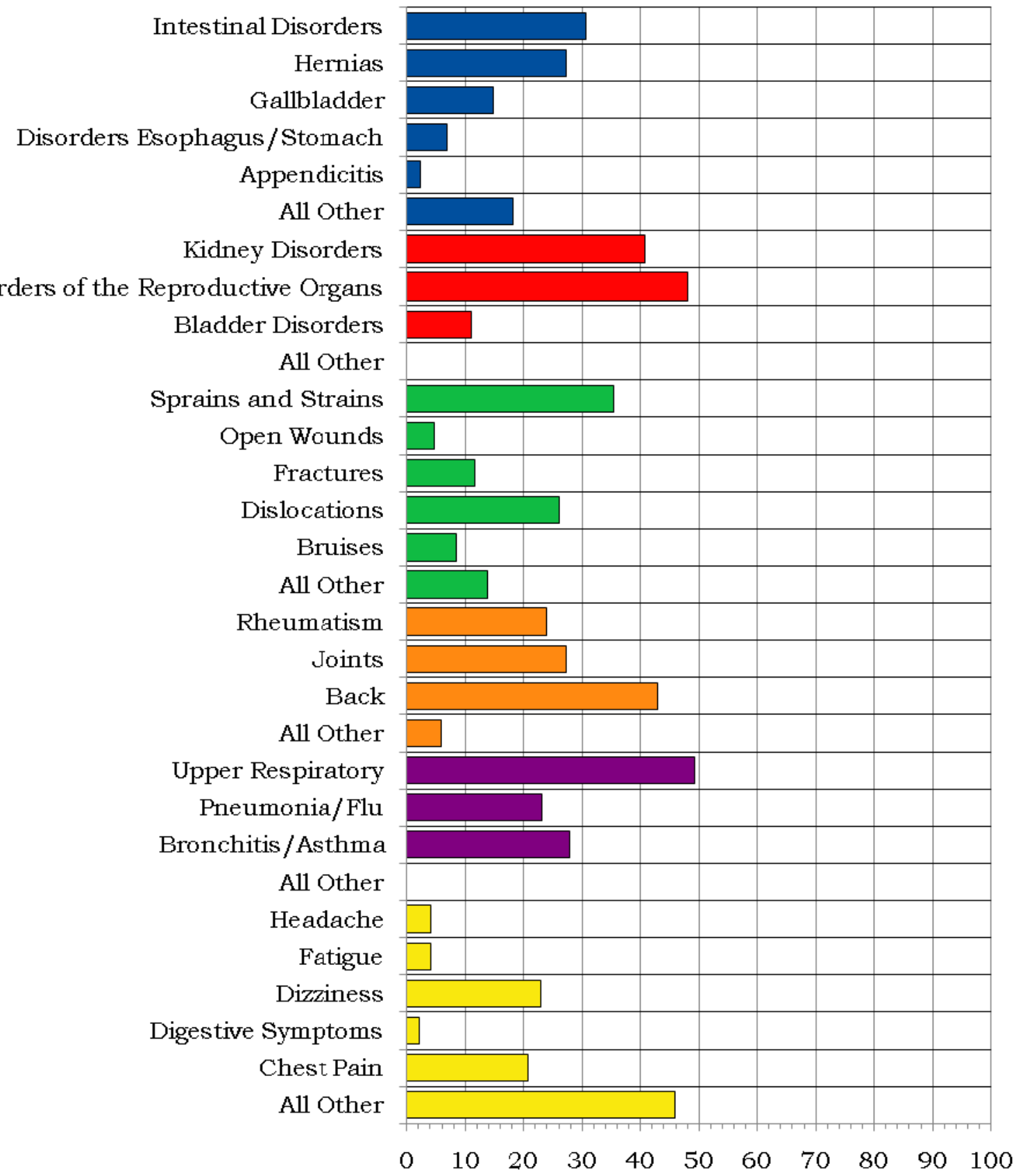

Percent Distribution of Diagnoses Within Diagnostic Category

Digestive, 88 Diagnoses

Genitourinary, 27 Diagnoses

Injury, 130 Diagnoses
Musculoskeletal, 121 Diagnoses

Respiratory, 126 Diagnoses

Unspecified Symptoms, 48 Diagnoses 


\section{Figure 10. Number of Most Frequently Reported Diagnoses by Job Category and Gender}

\begin{tabular}{|c|c|c|c|c|}
\hline Job Category & Men & & Womer & \\
\hline \multirow{4}{*}{ Professional } & Respiratory & 68 & Respiratory & 47 \\
\hline & Injury & 60 & Injury & 29 \\
\hline & M uscul oskeletal & 52 & M usculoskeletal & 20 \\
\hline & & & Digestive & 20 \\
\hline \multirow{3}{*}{ Administrative Support } & Respiratory & 1 & Respiratory & 50 \\
\hline & Heart/Circulatory & 1 & M usculoskeletal & 32 \\
\hline & & & Digestive & 26 \\
\hline \multirow{3}{*}{ Technical Support } & Injury & 24 & Respiratory & 14 \\
\hline & Respiratory & 20 & M usculoskeletal & 8 \\
\hline & Musculoskeletal & 11 & Digestive & 8 \\
\hline \multirow{5}{*}{ Service } & M usculoskeletal & 15 & M usculoskeletal & 16 \\
\hline & Respiratory & 9 & Injury & 10 \\
\hline & Digestive & 9 & Nervous System & 5 \\
\hline & Unspecified Symptoms & 9 & Psychological & 5 \\
\hline & & & Genitourinary & 5 \\
\hline \multirow{3}{*}{ Security and Fire } & M usculoskeletal & 5 & Respiratory & 1 \\
\hline & Respiratory & 3 & Injury & 1 \\
\hline & Injury & 3 & Cancer & 1 \\
\hline \multirow{3}{*}{ Crafts } & M usculoskeletal & 38 & M usculoskeletal & 4 \\
\hline & Injury & 37 & Nervous System & 2 \\
\hline & Respiratory & 24 & Psychological & 2 \\
\hline \multirow{5}{*}{ Line Operators } & Digestive & 3 & & \multirow[t]{5}{*}{0} \\
\hline & Injury & 2 & & \\
\hline & Respiratory & 1 & & \\
\hline & Heart/Circulatory & 1 & & \\
\hline & Benign Growths & 1 & & \\
\hline
\end{tabular}




\section{Rates of Disease Occurrence}

Figure 11. Rates for All Illnesses and Injuries Combined by Job Category, Gender, and Age

\begin{tabular}{|c|c|c|c|c|}
\hline \multirow{2}{*}{$\begin{array}{c}\text { All Illnesses \& } \\
\text { Injuries Combined }\end{array}$} & \multicolumn{4}{|c|}{ Rate per 1,000} \\
\hline & Job Category & Age & Men & Women \\
\hline & \multirow{2}{*}{ Professional } & $<50$ & 102 & 224 \\
\hline & & $50+$ & 207 & 337 \\
\hline & \multirow{2}{*}{ Administrative Support } & $<50$ & 154 & 480 \\
\hline & & $50+$ & 0 & 482 \\
\hline & \multirow{2}{*}{ Technical Support } & $<50$ & 302 & 464 \\
\hline & & $50+$ & 314 & 489 \\
\hline & \multirow{2}{*}{ Service } & $<50$ & 309 & 1,242 \\
\hline & & $50+$ & 771 & 606 \\
\hline & \multirow{2}{*}{ Security and Fire } & $<50$ & 310 & 200 \\
\hline & & $50+$ & 500 & 250 \\
\hline & \multirow{2}{*}{ Crafts } & $<50$ & 355 & 429 \\
\hline & & $50+$ & 476 & 875 \\
\hline & \multirow{2}{*}{ Line Operators } & $<50$ & 162 & 0 \\
\hline & & $50+$ & 118 & 0 \\
\hline
\end{tabular}

Figure 12. Rates for Selected Diagnostic Categories by Job Category, Gender, and Age

\begin{tabular}{|c|c|c|c|c|}
\hline \multirow{2}{*}{ Cancer } & \multicolumn{4}{|c|}{ Rate per 1,000} \\
\hline & Job Category & Age & Men & Women \\
\hline & Professional & $<50$ & 1 & 5 \\
\hline & & $50+$ & 8 & 10 \\
\hline & Administrative Sunport & $<50$ & 0 & 30 \\
\hline & & $50+$ & 0 & 14 \\
\hline & Technical Support & $<50$ & 5 & 0 \\
\hline & & $50+$ & 0 & 43 \\
\hline & Service & $<50$ & 0 & 0 \\
\hline & 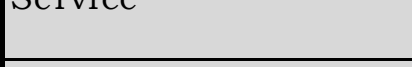 & $50+$ & 21 & 61 \\
\hline & Security and Fire & $<50$ & 0 & 0 \\
\hline & 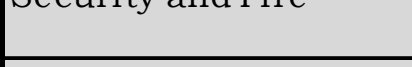 & $50+$ & 0 & 125 \\
\hline & Crafts & $<50$ & 6 & 0 \\
\hline & & $50+$ & 9 & 0 \\
\hline & Line $\mathrm{Or}$ & $<50$ & 0 & 0 \\
\hline & & $50+$ & 0 & 0 \\
\hline
\end{tabular}


Figure 12. Rates for Selected Diagnostic Categories by Job Category, Gender, and Age (Continued)

\begin{tabular}{|l|l|c|c|c|}
\hline \multirow{2}{*}{ Heart/Circulatory } & \multicolumn{4}{|c|}{ Rate per 1,000 } \\
\cline { 2 - 5 } & \multicolumn{1}{|c|}{ Job Category } & Age & M en & W omen \\
\cline { 2 - 5 } & Professional & $<50$ & $\mathbf{2}$ & $\mathbf{5}$ \\
\cline { 2 - 5 } & $50+$ & $\mathbf{1 6}$ & $\mathbf{3}$ \\
\hline \multirow{2}{*}{ Administrative Support } & $<50$ & $\mathbf{7 7}$ & $\mathbf{1 5}$ \\
\cline { 2 - 5 } & $50+$ & $\mathbf{0}$ & $\mathbf{3 6}$ \\
\cline { 2 - 5 } & \multirow{2}{*}{ Technical Support } & $<50$ & $\mathbf{2 6}$ & $\mathbf{1 4}$ \\
\cline { 2 - 5 } & & $50+$ & $\mathbf{1 0}$ & $\mathbf{2 1}$ \\
\cline { 2 - 5 } & \multirow{2}{*}{ Service } & $<50$ & $\mathbf{0}$ & $\mathbf{3 0}$ \\
\cline { 2 - 5 } & $50+$ & $\mathbf{1 0 4}$ & $\mathbf{0}$ \\
\cline { 2 - 5 } & \multirow{2}{*}{ Security and Fire } & $<50$ & $\mathbf{0}$ & $\mathbf{0}$ \\
\cline { 2 - 5 } & $50+$ & $\mathbf{0}$ & $\mathbf{0}$ \\
\cline { 2 - 5 } & \multirow{2}{*}{ Crafts } & $<50$ & $\mathbf{1 3}$ & $\mathbf{0}$ \\
\cline { 2 - 5 } & $50+$ & $\mathbf{3 9}$ & $\mathbf{0}$ \\
\cline { 2 - 5 } & \multirow{2}{*}{ Line Operators } & $<50$ & $\mathbf{0}$ & $\mathbf{0}$ \\
\cline { 2 - 5 } & $50+$ & $\mathbf{5 9}$ & $\mathbf{0}$ \\
\hline
\end{tabular}

\begin{tabular}{|c|c|c|c|c|}
\hline \multirow{2}{*}{ Respiratory } & \multicolumn{4}{|c|}{ Rate per 1,000} \\
\hline & Job Category & Age & Men & Women \\
\hline \multirow{14}{*}{ D } & \multirow{2}{*}{ Professional } & $<50$ & 20 & 54 \\
\hline & & $50+$ & 39 & 87 \\
\hline & \multirow{2}{*}{ Administrative Support } & $<50$ & 77 & 95 \\
\hline & & $50+$ & 0 & 112 \\
\hline & \multirow{2}{*}{ Technical Support } & $<50$ & 53 & 159 \\
\hline & & $50+$ & 95 & 64 \\
\hline & \multirow{2}{*}{ Service } & $<50$ & 43 & 30 \\
\hline & & $50+$ & 104 & 91 \\
\hline & \multirow{2}{*}{ Security and Fire } & $<50$ & 103 & 200 \\
\hline & & $50+$ & 0 & 0 \\
\hline & \multirow{2}{*}{ Crafts } & $<50$ & 52 & 0 \\
\hline & & $50+$ & 69 & 0 \\
\hline & \multirow{2}{*}{ Line Operators } & $<50$ & 27 & 0 \\
\hline & & $50+$ & 0 & 0 \\
\hline
\end{tabular}


Figure 12. Rates for Selected Diagnostic Categories by Job Category, Gender, and Age (Continued)

\begin{tabular}{|c|c|c|c|c|}
\hline \multirow{2}{*}{ Injury } & \multicolumn{4}{|c|}{ Rate per 1,000} \\
\hline & Job Category & Age & Men & Women \\
\hline & \multirow{2}{*}{ Professional } & $<50$ & 26 & 26 \\
\hline & & $50+$ & 27 & 63 \\
\hline & \multirow{2}{*}{ Administrative Support } & $<50$ & 0 & 35 \\
\hline & & $50+$ & 0 & 32 \\
\hline & \multirow{2}{*}{ Technical Support } & $<50$ & 106 & 43 \\
\hline & & $50+$ & 38 & 43 \\
\hline & \multirow{2}{*}{ Service } & $<50$ & 32 & 303 \\
\hline & & $50+$ & 21 & 0 \\
\hline & \multirow{2}{*}{ Security and Fire } & $<50$ & 69 & 0 \\
\hline & & $50+$ & 56 & 125 \\
\hline & \multirow{2}{*}{ Crafts } & $<50$ & 116 & 0 \\
\hline & & $50+$ & 82 & 0 \\
\hline & \multirow{2}{*}{ Line Operators } & $<50$ & 54 & 0 \\
\hline & & $50+$ & 0 & 0 \\
\hline
\end{tabular}

Time Trends

Figure 13. Age-Adjusted Rates for All Diagnoses Combined Among Women and Men from 2003 to 2008*

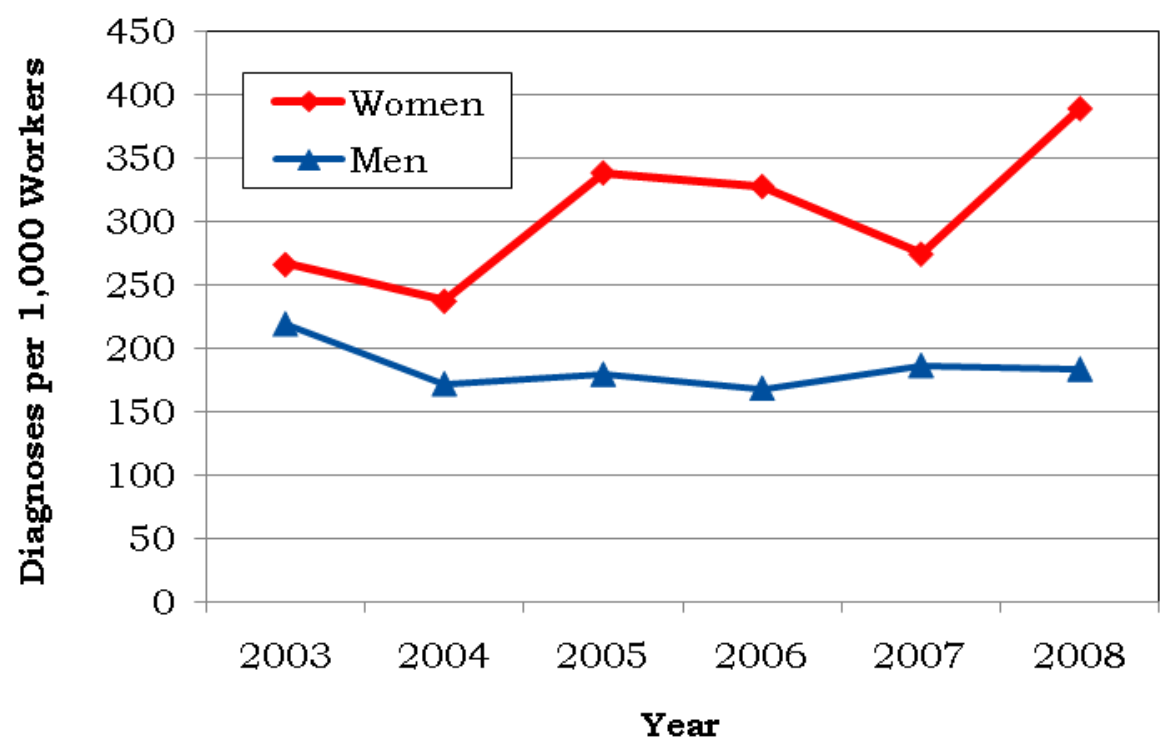

*Standardized to age distribution of 2000 U.S. population. 
Figure 14. Age-Adjusted Rates for Selected Diagnostic Categories Among Women and Men from 2003 to 2008*

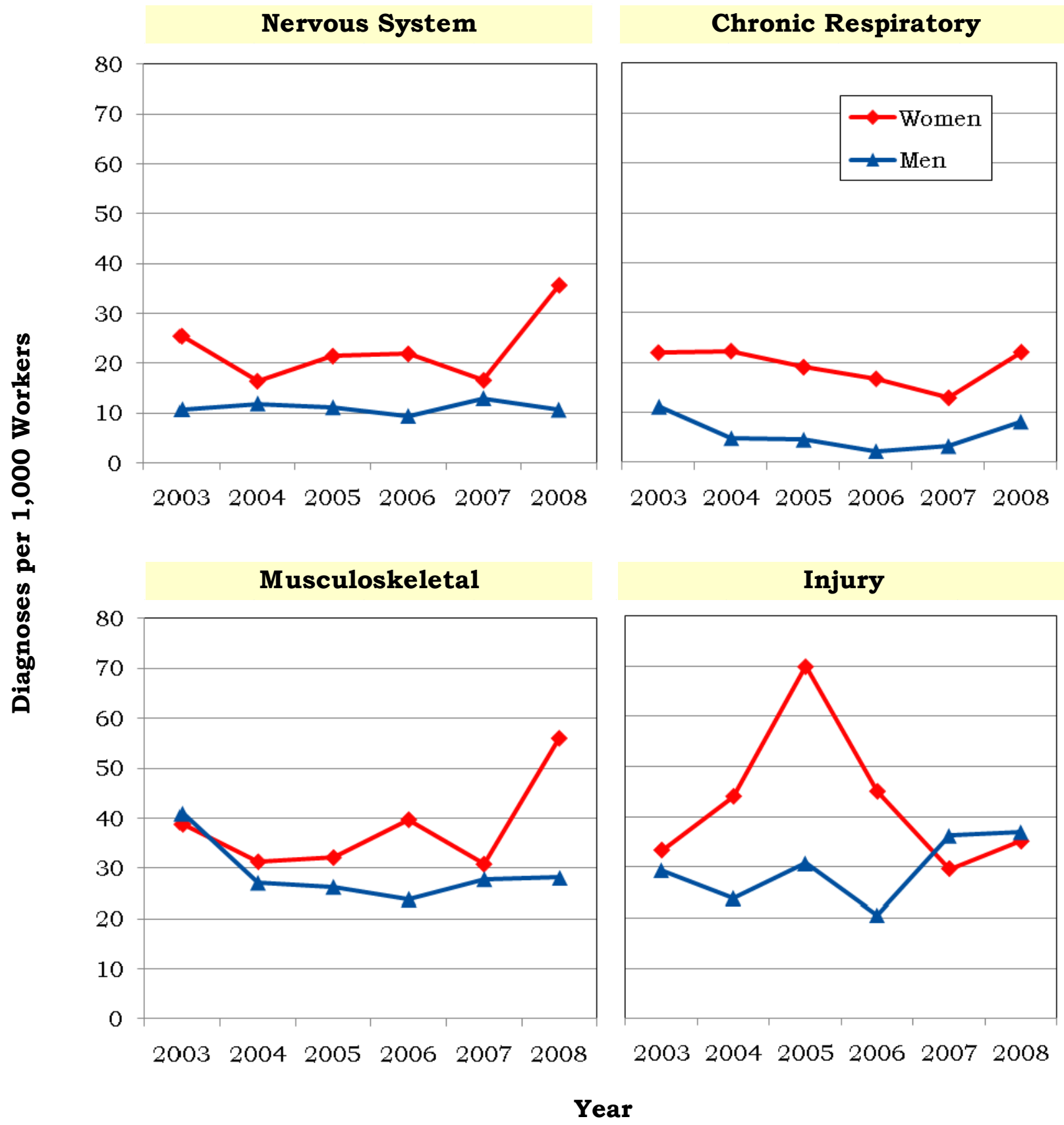

*Standardized to age distribution of 2000 U.S. population. 
Figure 15. Age-Adjusted Rates for All Diagnoses Combined Among Women and Men by Job Category from 2003 to 2008*

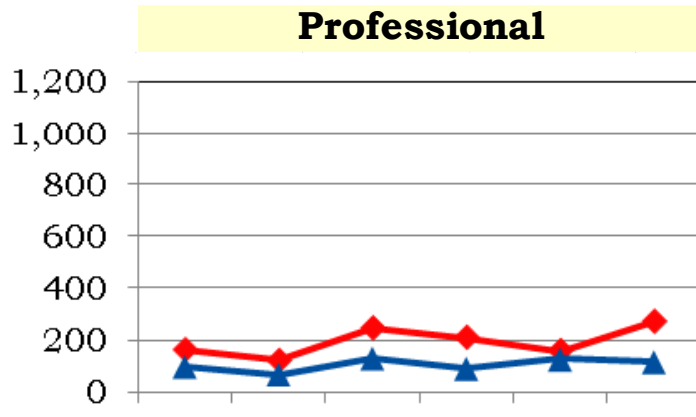

200320042005200620072008

Administrative Support

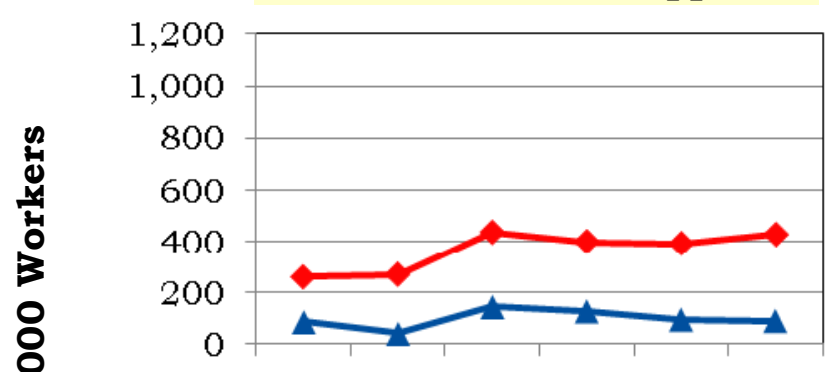

200320042005200620072008

Service

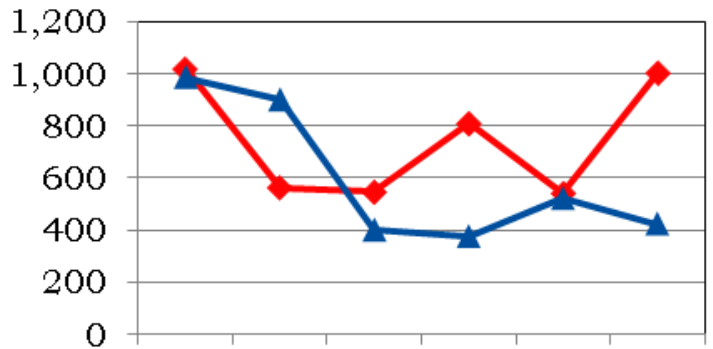

200320042005200620072008

Crafts

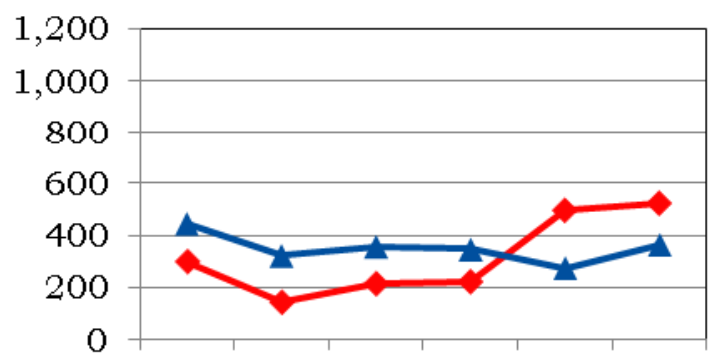

200320042005200620072008

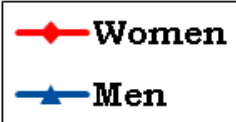

Technical Support

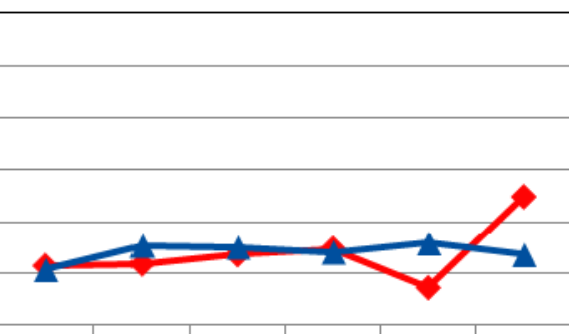

200320042005200620072008

Security and Fire

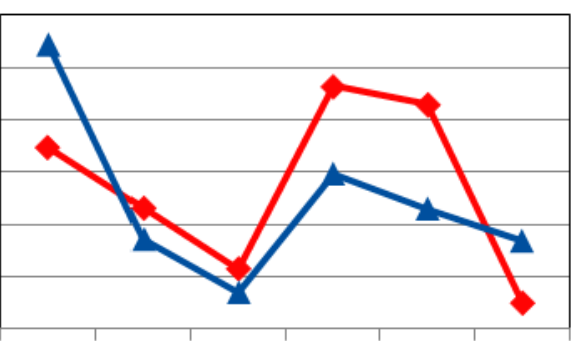

200320042005200620072008

Line Operators

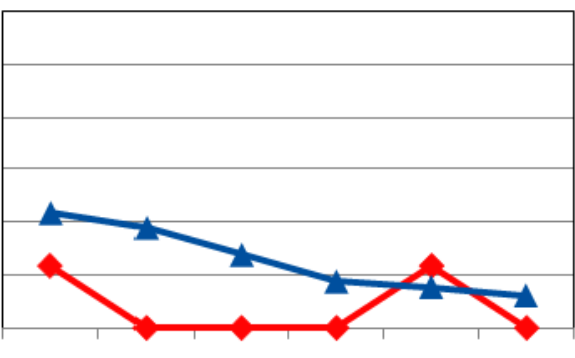

200320042005200620072008

Year

*Standardized to age distribution of 2000 U.S. population. 


\section{Sentinel Health Events for Occupations (SHEOs)}

An occupational sentinel health event (SHEO) is a disease, disability, or death that is likely to be occupationally related. Although sentinel health events may indicate an occupational exposure, many may result from nonoccupational exposures. Sentinel health events are therefore assessed in two categories:

Definite Sentinel Health Events: Diseases that are unlikely to occur in the absence of an occupational exposure (e.g., asbestosis).

Possible Sentinel Health Events: Diseases that may be occupational but can also occur in the absence of an occupational exposure (e.g., lung cancer or carpal tunnel syndrome).

Figure 16. Characteristics of SHEOs by Gender

\begin{tabular}{|l|c|c|c|c|}
\hline \multirow{2}{*}{} & \multicolumn{2}{|c|}{$\begin{array}{c}\text { Total Number of } \\
\text { SHEO Diagnoses }\end{array}$} & \multicolumn{2}{c|}{$\begin{array}{c}\text { Total Number of } \\
\text { Days Absent }\end{array}$} \\
\cline { 2 - 5 } & Men & Women & Men & W omen \\
\hline Definite & 0 & 0 & 0 & 0 \\
\hline Possible & 6 & 15 & 227 & 404 \\
\hline Total & 6 & 15 & 227 & 404 \\
\hline
\end{tabular}

Figure 17. SHEO Diagnoses by Gender

\begin{tabular}{|l|c|c|}
\hline \multirow{2}{*}{ Diagnoses } & \multicolumn{2}{c|}{ Gender } \\
\cline { 2 - 3 } & Women & M en \\
\hline Carpal Tunnel Syndrome & 13 & 3 \\
\hline M usculoskeletal Conditions & 0 & 1 \\
\hline Other Conditions & 2 & 2 \\
\hline
\end{tabular}


Occupational Safety and Health Administration (OSHA)-Recordable Events

Figure 18. OSHA-Recordable Events by Gender and Age

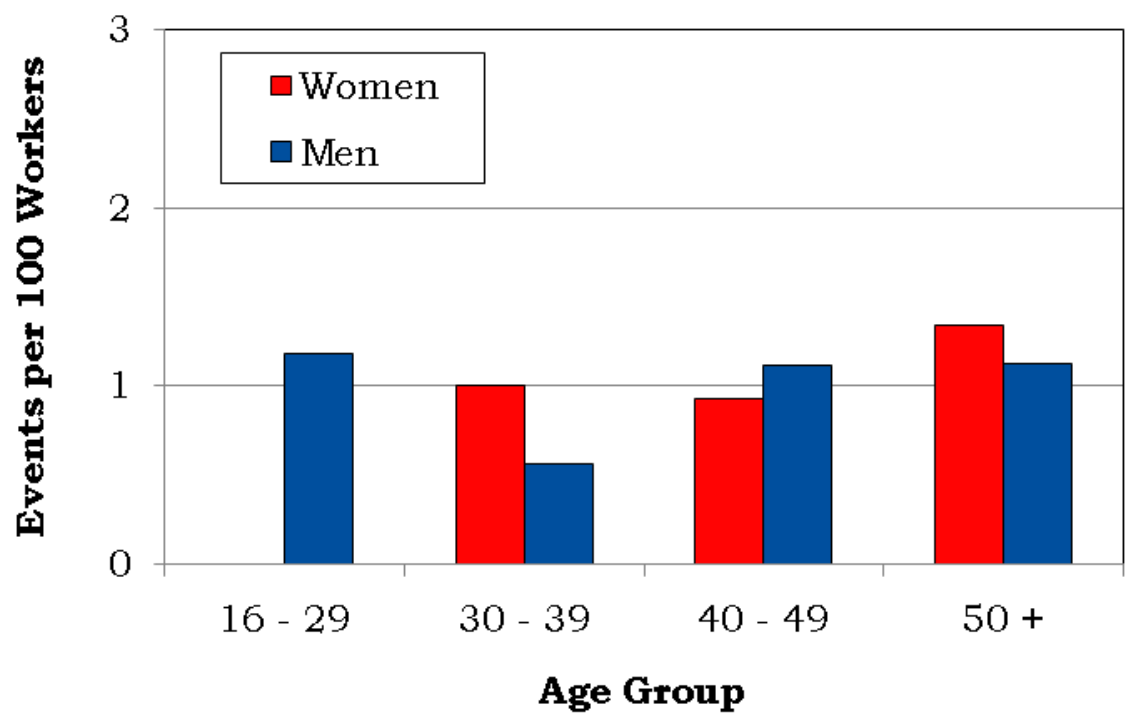

Figure 19. OSHA-Recordable Events by Job Category and Gender

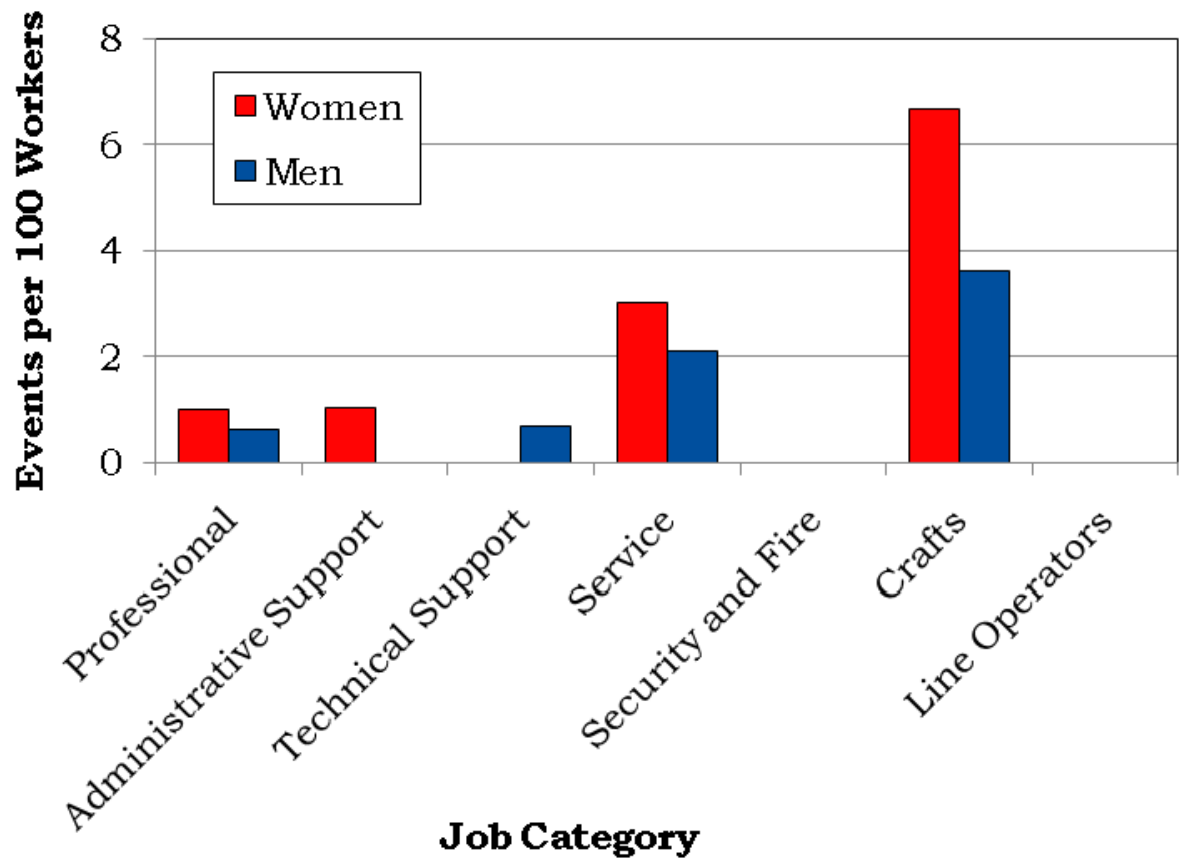




\section{Diagnostic and Accident Categories for OSHA-Recordable Events}

Figure 20. OSHA-Recordable Diagnoses by Diagnostic Category and Gender

\begin{tabular}{|l|c|c|}
\hline \multicolumn{1}{|c|}{ Diagnostic Category } & \multicolumn{2}{c|}{ Gender } \\
\cline { 2 - 3 } & Women & M en \\
\hline Musculoskeletal & $\mathbf{1 0}$ & $\mathbf{2 5}$ \\
\hline Respiratory & $\mathbf{1}$ & $\mathbf{0}$ \\
\hline Unspecified Symptoms & $\mathbf{4}$ & $\mathbf{4}$ \\
\hline Injury & $\mathbf{1 9}$ & $\mathbf{3 5}$ \\
\hline Fractures - Upper Limb & $\mathbf{3}$ & $\mathbf{1}$ \\
\hline Fractures - Lower Limb & $\mathbf{1}$ & $\mathbf{0}$ \\
\hline Back Sprains \& Strains & $\mathbf{1}$ & $\mathbf{3}$ \\
\hline Other Sprains \& Strains & $\mathbf{4}$ & $\mathbf{9}$ \\
\hline Open Wounds - Head, Neck, Trunk & $\mathbf{0}$ & $\mathbf{4}$ \\
\hline Open Wounds - Lower Limb & $\mathbf{0}$ & $\mathbf{1}$ \\
\hline Superficial Injuries & $\mathbf{0}$ & $\mathbf{1}$ \\
\hline Bruises & $\mathbf{5}$ & $\mathbf{4}$ \\
\hline Burns & $\mathbf{0}$ & $\mathbf{1}$ \\
\hline Injuries to Nerves \& Spinal Cord & $\mathbf{0}$ & $\mathbf{1}$ \\
\hline Unspecified Injuries & $\mathbf{4}$ & $\mathbf{9}$ \\
\hline Adverse Reactions to Non-Medical Substances & $\mathbf{1}$ & $\mathbf{1}$ \\
\hline
\end{tabular}


Figure 21. OSHA-Recordable Accidents by Type and Gender

\begin{tabular}{|l|c|c|}
\hline \multirow{2}{*}{ Accident Category } & \multicolumn{2}{c|}{ Gender } \\
\cline { 2 - 3 } & Women & M en \\
\cline { 2 - 3 } & $\begin{array}{c}\text { Number of } \\
\text { Accidents }\end{array}$ & $\begin{array}{c}\text { Number of } \\
\text { Accidents }\end{array}$ \\
\hline Motor Vehicle Traffic & $\mathbf{0}$ & $\mathbf{3}$ \\
\hline Poisoning - Non-Medicinal & $\mathbf{1}$ & $\mathbf{0}$ \\
\hline Falls & $\mathbf{9}$ & $\mathbf{4}$ \\
\hline Natural/Environmental Factors & $\mathbf{0}$ & $\mathbf{1}$ \\
\hline Other Accidents & $\mathbf{5}$ & $\mathbf{2 5}$ \\
\hline Struck by an Object & $\mathbf{2}$ & $\mathbf{7}$ \\
\hline Caught Between Objects & $\mathbf{0}$ & $\mathbf{1}$ \\
\hline Machinery & $\mathbf{1}$ & $\mathbf{0}$ \\
\hline Cutting/Piercing Instrument/Object & $\mathbf{0}$ & $\mathbf{1}$ \\
\hline Hot, Corrosive, or Caustic Material/Steam & $\mathbf{0}$ & $\mathbf{1}$ \\
\hline Overexertion/Strenuous Movements & $\mathbf{2}$ & $\mathbf{1 2}$ \\
\hline Repetitive Trauma & $\mathbf{0}$ & $\mathbf{3}$ \\
\hline Total & $\mathbf{1 5}$ & $\mathbf{3 3}$ \\
\hline
\end{tabular}




\section{Rates of OSHA-Recordable Events}

Figure 22. OSHA-Recordable Rates by Age and Job Categories Among Women, All Diagnoses Combined

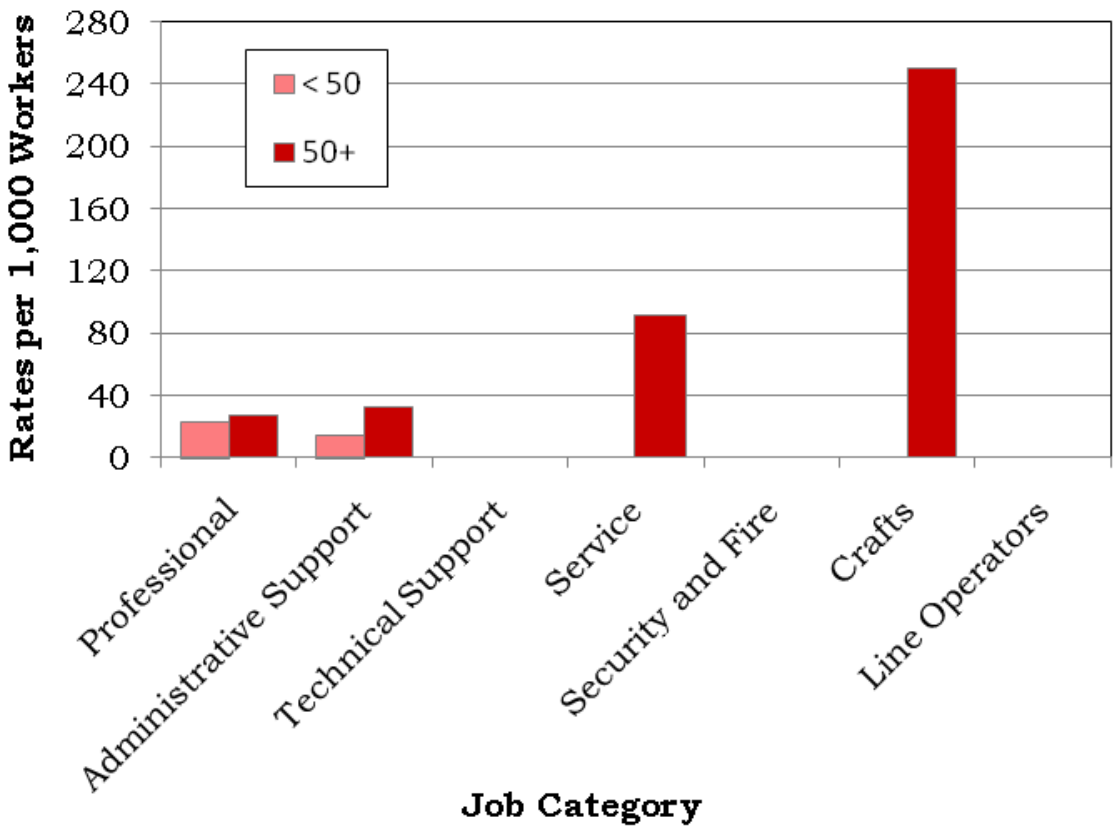

Figure 23. OSHA-Recordable Rates by Age and Job Categories Among Men, All Diagnoses Combined
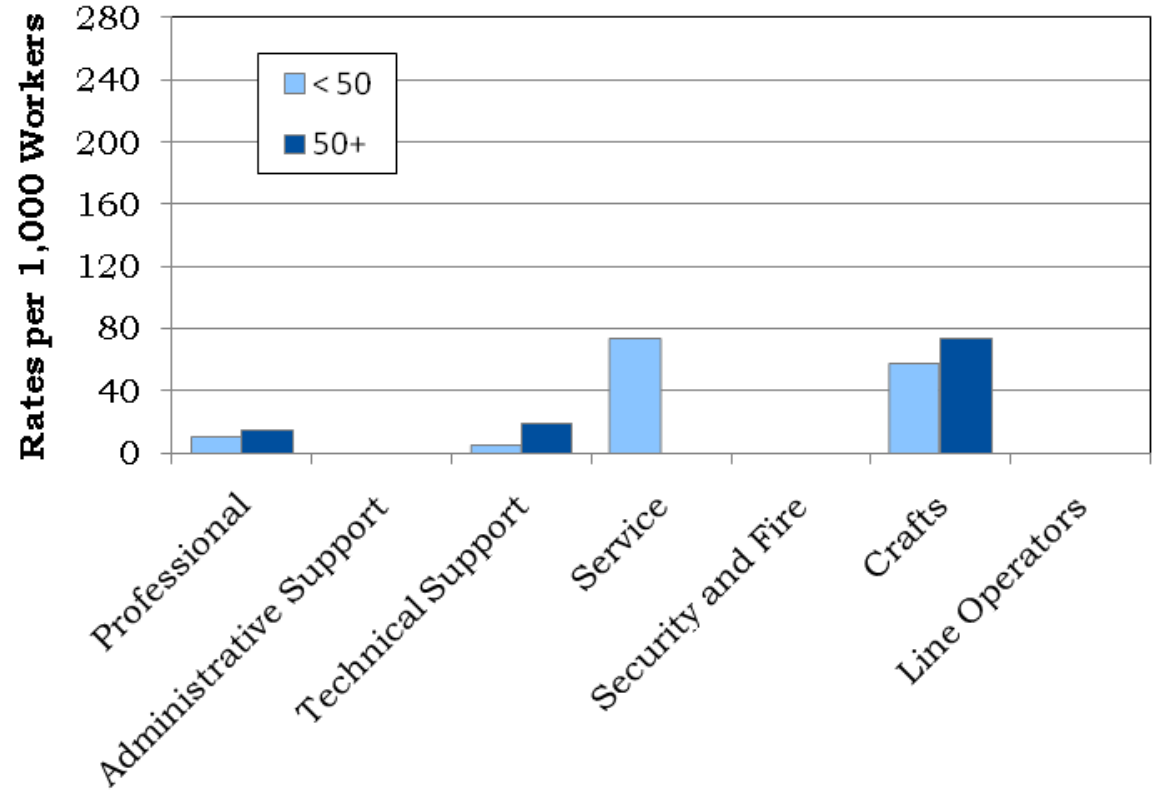

Job Category 


\section{Time Trends for OSHA-Recordable Events}

Figure 24. Age-Adjusted Rates for All OSHA-Recordable Diagnoses Combined Among Women and Men by Job Category from 2003 to 2008*

Professional

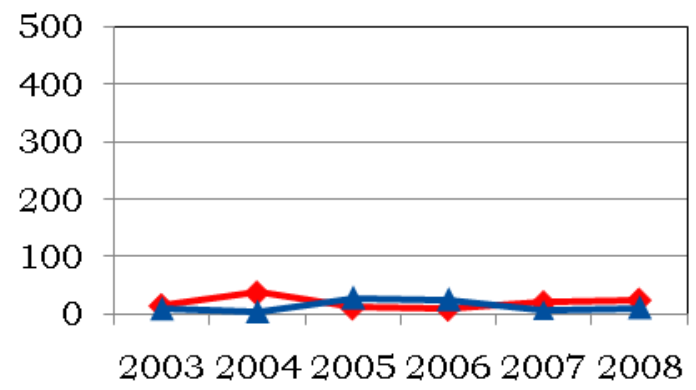

Administrative Support

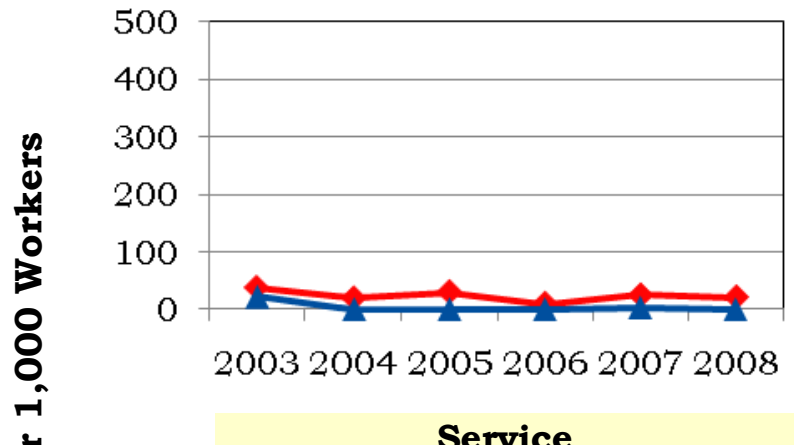

Service

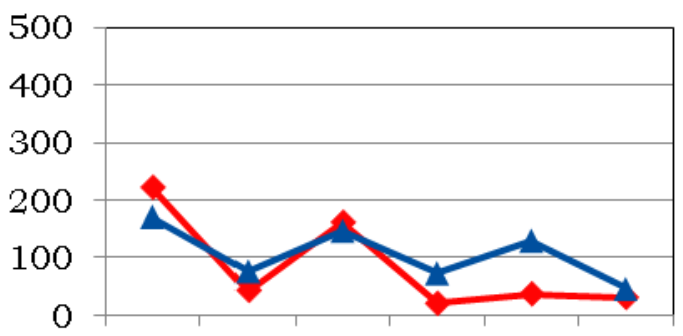

200320042005200620072008

Crafts

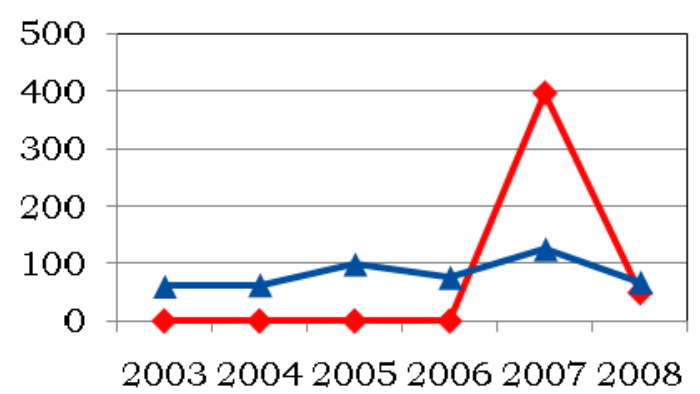

Technical Support

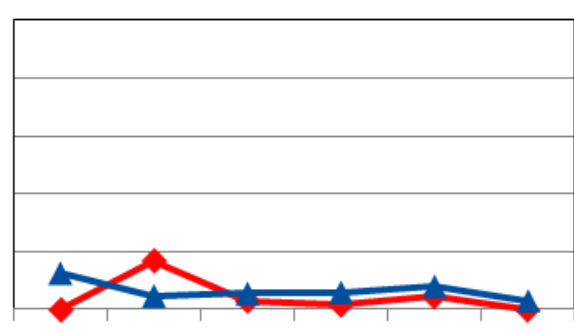

200320042005200620072008

Security and Fire

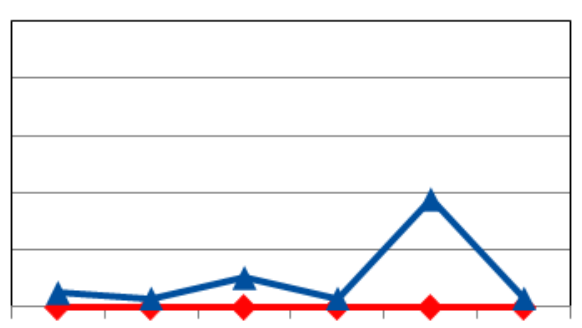

200320042005200620072008

Line Operators

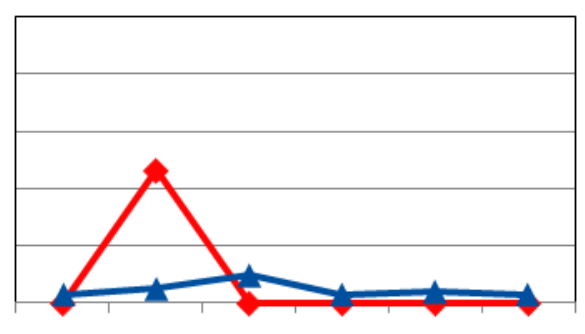

200320042005200620072008

Year

*Standardized to age distribution of 2000 U.S. population. 


\section{Appendices}


Oak Ridge National Laboratory 2008

Absence Data

Appendix A. Work Force by Gender, Age, and Job Category

\begin{tabular}{|c|c|c|c|c|c|c|c|c|c|c|c|}
\hline \multirow{3}{*}{ Job Category } & \multicolumn{5}{|c|}{ Women } & \multicolumn{5}{|c|}{ Men } & \multirow[b]{3}{*}{ TOTAL } \\
\hline & \multicolumn{4}{|c|}{ Age Group } & \multirow[b]{2}{*}{ TOTAL } & \multicolumn{4}{|c|}{ Age Group } & \multirow[b]{2}{*}{ TOTAL } & \\
\hline & $16-29$ & 30 - 39 & $40-49$ & $50+$ & & $16-29$ & $30-39$ & $40-49$ & $\mathbf{5 0 +}$ & & \\
\hline Professional & 46 & 108 & 238 & 300 & 692 & 92 & 368 & 636 & 1,179 & 2,275 & 2,967 \\
\hline Administrative Support & 20 & 58 & 122 & 278 & 478 & 6 & 2 & 5 & 10 & 23 & 501 \\
\hline Technical Support & 7 & 25 & 37 & 47 & 116 & 21 & 64 & 104 & 105 & 294 & 410 \\
\hline Service & 4 & 7 & 22 & 33 & 66 & 27 & 25 & 42 & 48 & 142 & 208 \\
\hline Security and Fire & 0 & 1 & 4 & 8 & 13 & 4 & 15 & 10 & 18 & 47 & 60 \\
\hline Crafts & 0 & 2 & 5 & 8 & 15 & 13 & 53 & 89 & 233 & 388 & 403 \\
\hline Line Operators & 0 & 0 & 1 & 0 & 1 & 7 & 13 & 17 & 17 & 54 & 55 \\
\hline TOTAL & 77 & 201 & 429 & 674 & 1,381 & 170 & 540 & 903 & 1,610 & 3,223 & 4,604 \\
\hline
\end{tabular}

Appendix B. Age Distribution of the Work Force by Gender

\begin{tabular}{|l|r|r|r|r|r|r|r|r|}
\hline \multirow{3}{*}{ Year } & \multicolumn{4}{|c|}{ Women } & \multicolumn{4}{c|}{ Men } \\
\cline { 2 - 9 } & \multicolumn{1}{|c|}{ Percent Distribution by Age Group } & \multicolumn{1}{c|}{ Percent Distribution by Age Group } \\
\cline { 2 - 9 } & $\mathbf{1 6}-\mathbf{2 9}$ & $\mathbf{3 0}-\mathbf{3 9}$ & $\mathbf{4 0}-\mathbf{4 9}$ & $\mathbf{5 0}+$ & $\mathbf{1 6}-\mathbf{2 9}$ & $\mathbf{3 0}-\mathbf{3 9}$ & $\mathbf{4 0}-\mathbf{4 9}$ & $\mathbf{5 0}+$ \\
\hline $\mathbf{1 9 9 9}$ & 3.31 & 24.96 & 44.75 & 26.98 & 2.72 & 18.64 & 42.04 & 36.60 \\
\hline $\mathbf{2 0 0 0}$ & 3.34 & 22.32 & 44.37 & 29.97 & 2.57 & 17.20 & 40.03 & 40.20 \\
\hline $\mathbf{2 0 0 1}$ & 3.49 & 19.15 & 43.57 & 33.80 & 1.70 & 15.81 & 39.34 & 43.15 \\
\hline $\mathbf{2 0 0 2}$ & 2.16 & 19.68 & 44.02 & 34.14 & 1.82 & 15.47 & 38.48 & 44.23 \\
\hline $\mathbf{2 0 0 3}$ & 2.05 & 17.95 & 40.90 & 39.10 & 2.33 & 14.95 & 35.36 & 47.36 \\
\hline $\mathbf{2 0 0 4}$ & 2.89 & 16.85 & 39.88 & 40.38 & 2.55 & 14.57 & 35.71 & 47.16 \\
\hline $\mathbf{2 0 0 5}$ & 4.01 & 14.97 & 38.19 & 42.82 & 3.48 & 14.40 & 33.13 & 48.99 \\
\hline $\mathbf{2 0 0 6}$ & 3.69 & 14.82 & 35.14 & 46.35 & 4.39 & 14.96 & 31.32 & 49.33 \\
\hline $\mathbf{2 0 0 7}$ & 5.11 & 14.31 & 32.84 & 47.74 & 4.80 & 15.39 & 29.86 & 49.95 \\
\hline $\mathbf{2 0 0 8}$ & 5.58 & 14.55 & 31.06 & 48.81 & 5.27 & 16.75 & 28.02 & 49.95 \\
\hline
\end{tabular}


Oak Ridge National Laboratory 2008

Absence Data

Appendix C. Total Number of Workers Who Reported at Least One Absence by Gender, Age, and Job Category*

\begin{tabular}{|c|c|c|c|c|c|c|c|c|c|c|c|}
\hline \multirow{3}{*}{ Job Category } & \multicolumn{5}{|c|}{ Women } & \multicolumn{5}{|c|}{ Men } & \multirow[b]{3}{*}{ TOTAL } \\
\hline & \multicolumn{4}{|c|}{ Age Group } & \multirow[b]{2}{*}{ TOTAL } & \multicolumn{4}{|c|}{ Age Group } & \multirow[b]{2}{*}{ TOTAL } & \\
\hline & $16-29$ & 30 - 39 & $40-49$ & $50+$ & & $16-29$ & 30 - 39 & $40-49$ & $50+$ & & \\
\hline Professional & 11 & 10 & 32 & 60 & 113 & 3 & 21 & 52 & 149 & 225 & 338 \\
\hline Administrative Support & 2 & 14 & 29 & 73 & 118 & 0 & 0 & 2 & 0 & 2 & 120 \\
\hline Technical Support & 2 & 6 & 12 & 12 & 32 & 4 & 10 & 19 & 23 & 56 & 88 \\
\hline Service & 3 & 1 & 11 & 12 & 27 & 3 & 2 & 10 & 21 & 36 & 63 \\
\hline Security and Fire & 0 & 0 & 1 & 1 & 2 & 2 & 5 & 1 & 5 & 13 & 15 \\
\hline Crafts & 0 & 2 & 0 & 3 & 5 & 2 & 14 & 15 & 67 & 98 & 103 \\
\hline Line Operators & 0 & 0 & 0 & 0 & 0 & 0 & 1 & 3 & 2 & 6 & 6 \\
\hline TOTAL & 18 & 33 & 85 & 161 & 297 & 14 & 53 & 102 & 267 & 436 & 733 \\
\hline
\end{tabular}

*Only those job categories and gender/age combinations with at least one absence appear in this table.

Appendix D. Total Number of Absences by Gender, Age, and Job Category*

\begin{tabular}{|c|c|c|c|c|c|c|c|c|c|c|c|}
\hline \multirow{3}{*}{ Job Category } & \multicolumn{5}{|c|}{ Women } & \multicolumn{5}{|c|}{ Men } & \multirow[b]{3}{*}{ TOTAL } \\
\hline & \multicolumn{4}{|c|}{ Age Group } & \multirow[b]{2}{*}{ TOTAL } & \multicolumn{4}{|c|}{ Age Group } & \multirow[b]{2}{*}{ TOTAL } & \\
\hline & $16-29$ & 30 - 39 & $40-49$ & $50+$ & & $16-29$ & 30 - 39 & $40-49$ & $50+$ & & \\
\hline Professional & 13 & 15 & 38 & 70 & 136 & 3 & 25 & 60 & 183 & 271 & 407 \\
\hline Administrative Support & 2 & 24 & 37 & 102 & 165 & 0 & 0 & 2 & 0 & 2 & 167 \\
\hline Technical Support & 4 & 8 & 14 & 16 & 42 & 5 & 11 & 24 & 27 & 67 & 109 \\
\hline Service & 4 & 3 & 18 & 17 & 42 & 4 & 3 & 12 & 29 & 48 & 90 \\
\hline Security and Fire & 0 & 0 & 1 & 2 & 3 & 2 & 6 & 1 & 7 & 16 & 19 \\
\hline Crafts & 0 & 2 & 0 & 4 & 6 & 2 & 19 & 20 & 89 & 130 & 136 \\
\hline Line Operators & 0 & 0 & 0 & 0 & 0 & 0 & 1 & 4 & 2 & 7 & 7 \\
\hline TOTAL & 23 & 52 & 108 & 211 & 394 & 16 & 65 & 123 & 337 & 541 & 935 \\
\hline
\end{tabular}

*Only those job categories and gender/age combinations with at least one absence appear in this table. 
Oak Ridge National Laboratory 2008

Absence Data

Appendix E. Distribution of the Number of Calendar Days Missed per Absence by Gender and Age*

\begin{tabular}{|c|c|c|c|c|c|c|c|c|c|c|c|}
\hline \multirow{3}{*}{$\begin{array}{c}\text { Number of } \\
\text { Calendar Days }\end{array}$} & \multicolumn{5}{|c|}{ Women } & \multicolumn{5}{|c|}{ Men } & \multirow[b]{3}{*}{ TOTAL } \\
\hline & \multicolumn{4}{|c|}{ Age Group } & \multirow[b]{2}{*}{ TOTAL } & \multicolumn{4}{|c|}{ Age Group } & \multirow[b]{2}{*}{ TOTAL } & \\
\hline & $16-29$ & 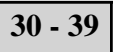 & 40 - 49 & $50+$ & & $16-29$ & $30-39$ & 40 - 49 & $50+$ & & \\
\hline$<15$ & 22 & 36 & 64 & 133 & 255 & 12 & 45 & 83 & 207 & 347 & 602 \\
\hline $15-28$ & 1 & 7 & 19 & 30 & 57 & 4 & 8 & 18 & 51 & 81 & 138 \\
\hline $29-42$ & 0 & 5 & 8 & 13 & 26 & 0 & 7 & 7 & 21 & 35 & 61 \\
\hline $43-56$ & 0 & 0 & 11 & 11 & 22 & 0 & 2 & 5 & 15 & 22 & 44 \\
\hline $57-91$ & 0 & 3 & 2 & 14 & 19 & 0 & 2 & 4 & 18 & 24 & 43 \\
\hline $92-182$ & 0 & 1 & 4 & 9 & 14 & 0 & 1 & 6 & 23 & 30 & 44 \\
\hline $183+$ & 0 & 0 & 0 & 1 & 1 & 0 & 0 & 0 & 2 & 2 & 3 \\
\hline TOTAL & 23 & 52 & 108 & 211 & 394 & 16 & 65 & 123 & 337 & 541 & 935 \\
\hline
\end{tabular}

*Only those gender/age combinations with at least one absence appear in this table. 
Oak Ridge National Laboratory 2008

Absence Data

Appendix F. Distribution of the Number of Calendar Days Missed per Absence by Gender and Job Category*

Women

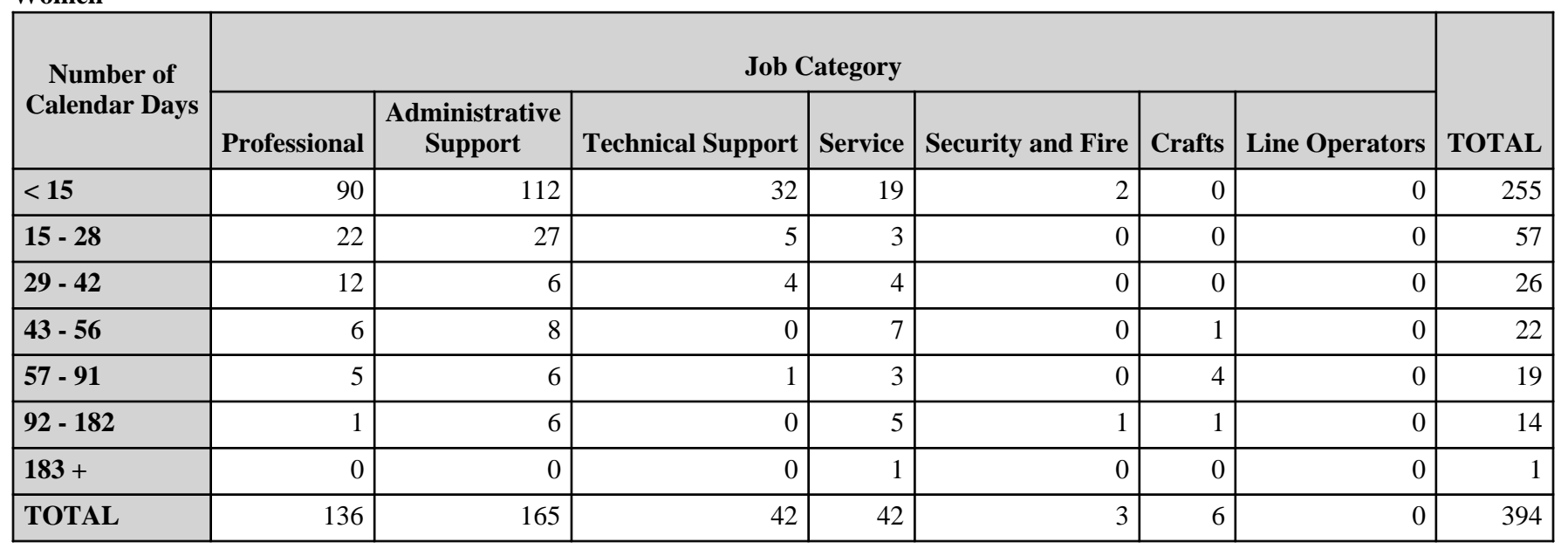

Men

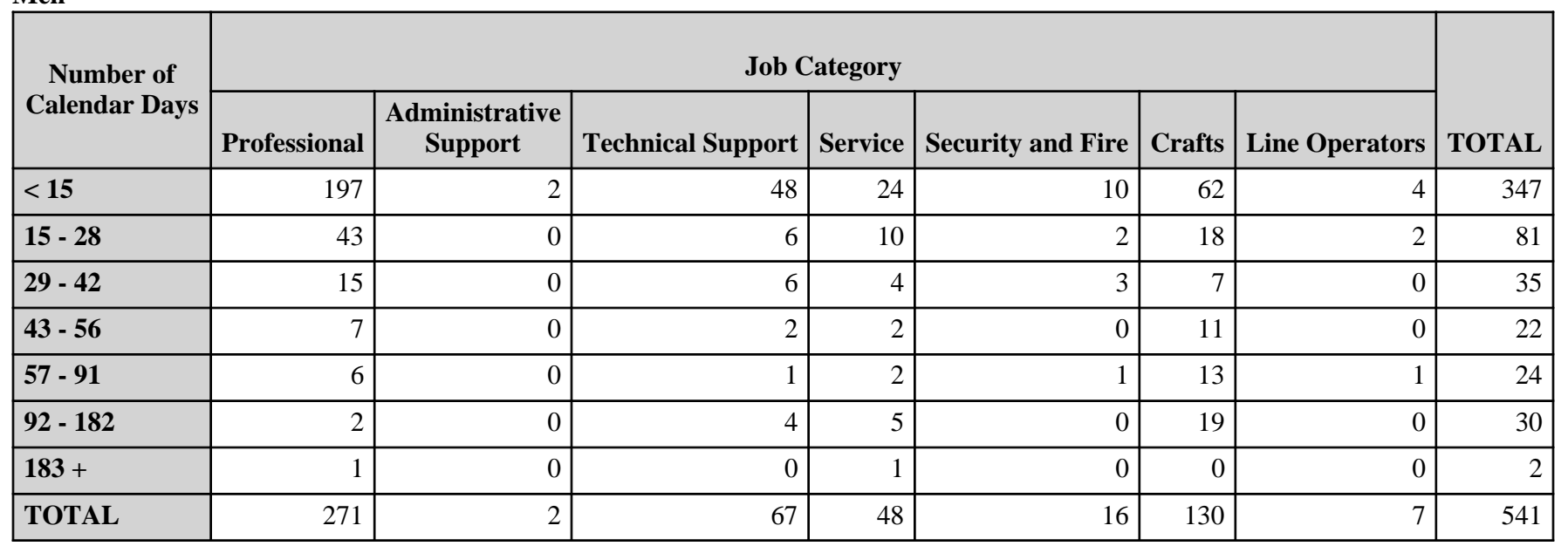

*Only those gender/job category combinations with at least one absence appear in this table. 
Oak Ridge National Laboratory 2008

Absence Data

Appendix G. Number of Diagnoses in Each Diagnostic Category by Gender and Age*

\begin{tabular}{|c|c|c|c|c|c|c|}
\hline & & \multicolumn{5}{|c|}{ Women } \\
\hline & & \multicolumn{4}{|c|}{ Age Group } & \multirow[b]{2}{*}{ TOTAL } \\
\hline & & $16-29$ & $30-39$ & $40-49$ & $\mathbf{5 0 +}$ & \\
\hline Diagnostic Category & ICD-9-CM Code & \multirow[b]{2}{*}{0} & \multirow[b]{2}{*}{8} & \multirow[b]{2}{*}{4} & \multirow[b]{2}{*}{16} & \multirow[b]{2}{*}{28} \\
\hline INFECTIOUS \& PARASITIC DISEASES (DIS) & \begin{tabular}{|c|}
$001-139$ \\
\end{tabular} & & & & & \\
\hline -Intestinal Infectious Dis & 001-009 & 0 & 0 & 0 & 2 & 2 \\
\hline -Other Bacterial Dis & 030-041 & 0 & 6 & 0 & 4 & 10 \\
\hline -Polio \& Other Viral CNS Dis & 045-049 & 0 & 0 & 0 & 0 & 0 \\
\hline -Viral Dis with Exanthem & $050-057$ & 0 & 1 & 0 & 3 & 4 \\
\hline -Other Viral Dis \& Chlamydiae & 070-079 & 0 & 1 & 4 & 6 & 11 \\
\hline -Mycoses & $110-118$ & 0 & 0 & 0 & 1 & 1 \\
\hline MALIGNANT NEOPLASMS & 140-208, 230-234 & 0 & 6 & 2 & 12 & 20 \\
\hline -Digestive \& Peritoneal & \begin{tabular}{|l|}
$150-159$ \\
\end{tabular} & 0 & 0 & 0 & 0 & 0 \\
\hline -Respiratory \& Intrathoracic & $160-165$ & 0 & 0 & 0 & 1 & 1 \\
\hline -Bone, Connective Tissue, Skin & 170-173, 176 & 0 & 0 & 0 & 3 & 3 \\
\hline -Breast & \begin{tabular}{|l|}
$174-175$ \\
\end{tabular} & 0 & 5 & 1 & 3 & 9 \\
\hline -Genitourinary & 179-189 & 0 & 0 & 0 & 1 & 1 \\
\hline -Other \& Unspecified Sites & 190, 193-199 & 0 & 1 & 1 & 1 & 3 \\
\hline -Lymphatic \& Hematopoietic & $200-208$ & 0 & 0 & 0 & 0 & 0 \\
\hline -Carcinoma in situ & $230-234$ & 0 & 0 & 0 & 3 & 3 \\
\hline BENIGN \& UNCERTAIN NEOPLASMS & 210-229, 235-239 & 0 & 2 & 7 & 7 & 16 \\
\hline ENDOCRINE/METABOLIC/IMMUNITY & $240-279$ & 0 & 1 & 4 & 6 & 11 \\
\hline -Thyroid Gland Disorders & $240-246$ & 0 & 0 & 2 & 2 & 4 \\
\hline -Other Endocrine Gland Dis & $250-259$ & 0 & 0 & 0 & 2 & 2 \\
\hline -Other Metabolic \& Immunity Disorders & 270-279 & 0 & 1 & 2 & 2 & 5 \\
\hline BLOOD \& BLOOD-FORMING ORGANS & $280-289$ & 0 & 1 & 0 & 1 & 2 \\
\hline MENTAL DISORDERS & $290-319$ & 0 & 3 & 7 & 1 & 11 \\
\hline -Psychoses & $290-299$ & 0 & 0 & 0 & 0 & 0 \\
\hline -Non-Psychotic Disorders & $300-302,306-316$ & 0 & 3 & 7 & 1 & 11 \\
\hline -Alcohol Dependence & 303 & 0 & 0 & 0 & 0 & 0 \\
\hline NERVOUS SYSTEM (NS) \& SENSE ORGANS & $320-389$ & 6 & 3 & 5 & 26 & 40 \\
\hline -Inflammatory Dis of Central NS & $320-326$ & 1 & 0 & 0 & 0 & 1 \\
\hline -Hereditary/Degenerative Central NS Dis & 330-337 & 0 & 0 & 0 & 1 & 1 \\
\hline -Other Disorders of Central NS & $340-349$ & 1 & 1 & 3 & 2 & 7 \\
\hline -Disorders of Peripheral NS & $350-359$ & 0 & 1 & 0 & 16 & 17 \\
\hline -Disorders of Eye & $360-379$ & 3 & 0 & 2 & 6 & 11 \\
\hline -Dis of Ear \& Mastoid & 380-389 & 1 & 1 & 0 & 1 & 3 \\
\hline CIRCULATORY SYSTEM & $390-459$ & 0 & 1 & 6 & 12 & 19 \\
\hline -Hypertensive Dis & $401-405$ & 0 & 0 & 1 & 1 & 2 \\
\hline -Ischemic Heart Dis & $410-414$ & 0 & 0 & 1 & 2 & 3 \\
\hline -Dis of Pulmonary Circulation & $415-417$ & 0 & 0 & 0 & 0 & 0 \\
\hline
\end{tabular}

*Only those diagnostic categories and gender/age combinations with at least one occurrence appear in this table. 
Oak Ridge National Laboratory 2008

Absence Data

Appendix G. Number of Diagnoses in Each Diagnostic Category by Gender and Age*

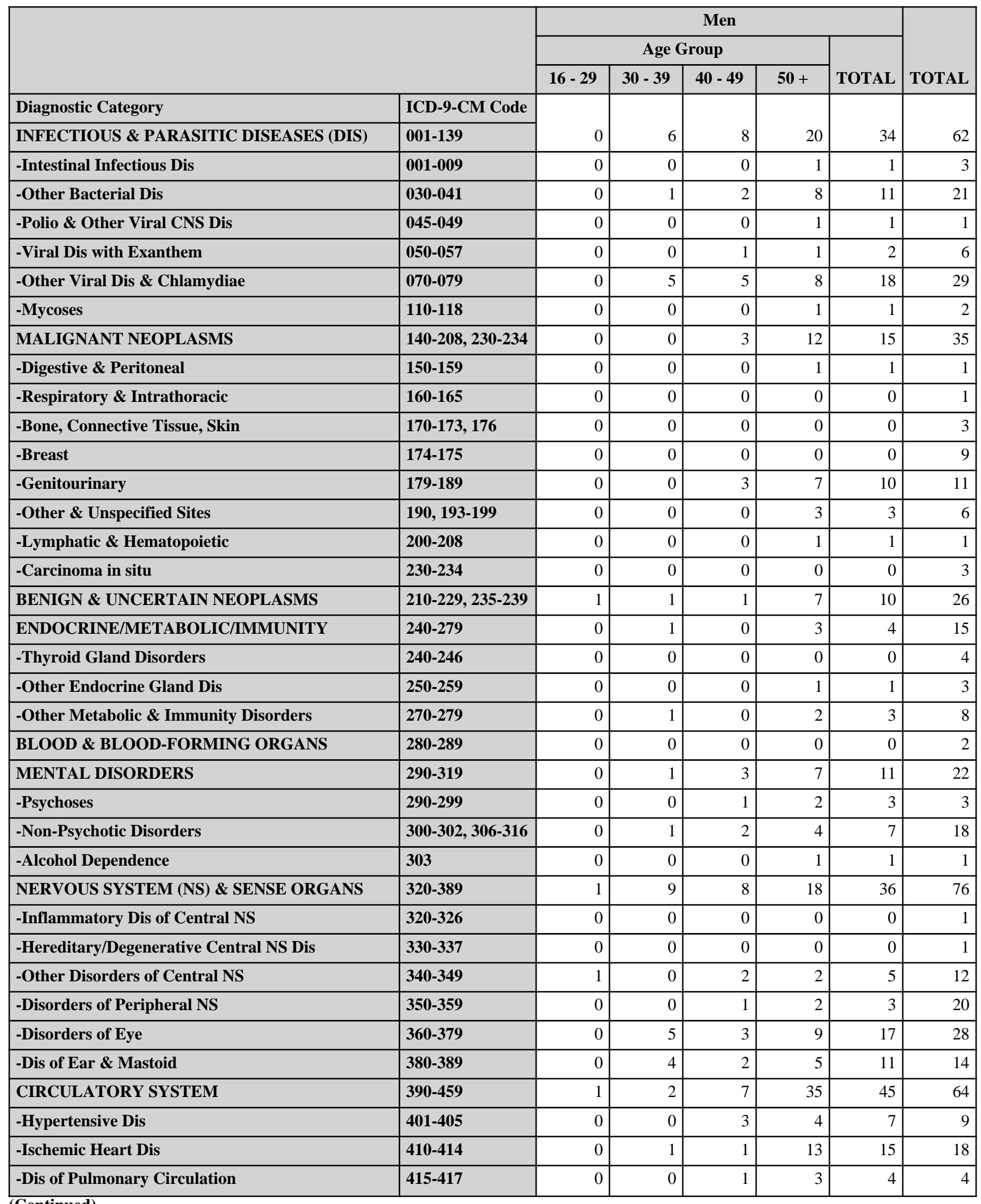

(Continued)

*Only those diagnostic categories and gender/age combinations with at least one occurrence appear in this table. 
Oak Ridge National Laboratory 2008

Absence Data

Appendix G. Number of Diagnoses in Each Diagnostic Category by Gender and Age*

\begin{tabular}{|c|c|c|c|c|c|c|}
\hline & & \multicolumn{5}{|c|}{ Women } \\
\hline & & \multicolumn{4}{|c|}{ Age Group } & \multirow[b]{2}{*}{ TOTAL } \\
\hline & & $16-29$ & 30 - 39 & $40-49$ & $50+$ & \\
\hline Diagnostic Category & ICD-9-CM Code & \multirow[b]{2}{*}{0} & \multirow[b]{2}{*}{0} & \multirow[b]{2}{*}{1} & \multirow[b]{2}{*}{0} & \multirow[b]{2}{*}{1} \\
\hline -Other Heart Dis & $420-429$ & & & & & \\
\hline -Cerebrovascular Dis & $430-438$ & 0 & 0 & 2 & 1 & 3 \\
\hline -Dis of Arteries \& Capillaries & $440-448$ & 0 & 0 & 0 & 0 & 0 \\
\hline -Dis of Veins, Lymphatics, Other & $451-459$ & 0 & 1 & 1 & 8 & 10 \\
\hline RESPIRATORY SYSTEM & $460-519$ & 9 & 19 & 25 & 63 & 116 \\
\hline -Acute Respiratory Infections & $460-466$ & 4 & 4 & 6 & 11 & 25 \\
\hline -Other Dis Upper Respiratory Tract & $470-478$ & 1 & 7 & 2 & 20 & 30 \\
\hline -Pneumonia \& Influenza & $480-487$ & 2 & 2 & 10 & 12 & 26 \\
\hline -Chronic Obstructive Dis & $490-496$ & 2 & 4 & 7 & 19 & 32 \\
\hline -Other Respiratory Dis & $510-519$ & 0 & 2 & 0 & 1 & 3 \\
\hline DIGESTIVE SYSTEM & $520-579$ & 6 & 11 & 14 & 26 & 57 \\
\hline -Oral Cavity, Saliva Glands, Jaw & $520-529$ & 4 & 0 & 1 & 3 & 8 \\
\hline -Esophagus, Stomach, Duodenum & $530-537$ & 0 & 4 & 1 & 3 & 8 \\
\hline -Appendicitis & $540-543$ & 0 & 0 & 1 & 0 & 1 \\
\hline -Hernia & $550-553$ & 0 & 1 & 1 & 2 & 4 \\
\hline -Enteritis, Colitis & $555-558$ & 2 & 3 & 0 & 8 & 13 \\
\hline -Other Intestinal Dis & $560-569$ & 0 & 2 & 5 & 4 & 11 \\
\hline -Other Digestive Dis & $570-579$ & 0 & 1 & 5 & 6 & 12 \\
\hline GENITOURINARY SYSTEM & $580-629$ & 3 & 1 & 11 & 23 & 38 \\
\hline -Nephritis, Nephrosis & $580-589$ & 0 & 0 & 0 & 1 & 1 \\
\hline -Other Urinary Dis & $590-599$ & 0 & 1 & 2 & 12 & 15 \\
\hline -Male Genital Organ Dis & $600-608$ & 0 & 0 & 0 & 0 & 0 \\
\hline -Breast Disorders & $610-611$ & 1 & 0 & 0 & 0 & 1 \\
\hline -Pelvic Inflammatory Dis & 614-616 & 0 & 0 & 1 & 0 & 1 \\
\hline -Other Female Disorders & $617-629$ & 2 & 0 & 8 & 10 & 20 \\
\hline PREGNANCY \& CHILDBIRTH & $630-677$ & 1 & 2 & 0 & 0 & 3 \\
\hline -Pregnancy with Abortive Outcome & 634-639 & 1 & 2 & 0 & 0 & 3 \\
\hline SKIN \& SUBCUTANEOUS TISSUE & $680-709$ & 0 & 0 & 3 & 3 & 6 \\
\hline -Infections & $680-686$ & 0 & 0 & 2 & 1 & 3 \\
\hline -Other Inflammatory Conditions & $690-698$ & 0 & 0 & 0 & 1 & 1 \\
\hline -Other & 700-709 & 0 & 0 & 1 & 1 & 2 \\
\hline $\begin{array}{l}\text { MUSCULOSKELETAL \& CONNECTIVE } \\
\text { TISSUE }\end{array}$ & $710-739$ & 4 & 13 & 25 & 38 & 80 \\
\hline -Arthropathies & $710-719$ & 0 & 3 & 8 & 12 & 23 \\
\hline -Dorsopathies & $720-724$ & 4 & 8 & 8 & 7 & 27 \\
\hline -Rheumatism, Excluding Back & 725-729 & 0 & 2 & 7 & 15 & 24 \\
\hline -Other Dis \& Acquired Deformities & $730-739$ & 0 & 0 & 2 & 4 & 6 \\
\hline
\end{tabular}

(Continued)

*Only those diagnostic categories and gender/age combinations with at least one occurrence appear in this table. 
Oak Ridge National Laboratory 2008

Absence Data

Appendix G. Number of Diagnoses in Each Diagnostic Category by Gender and Age*

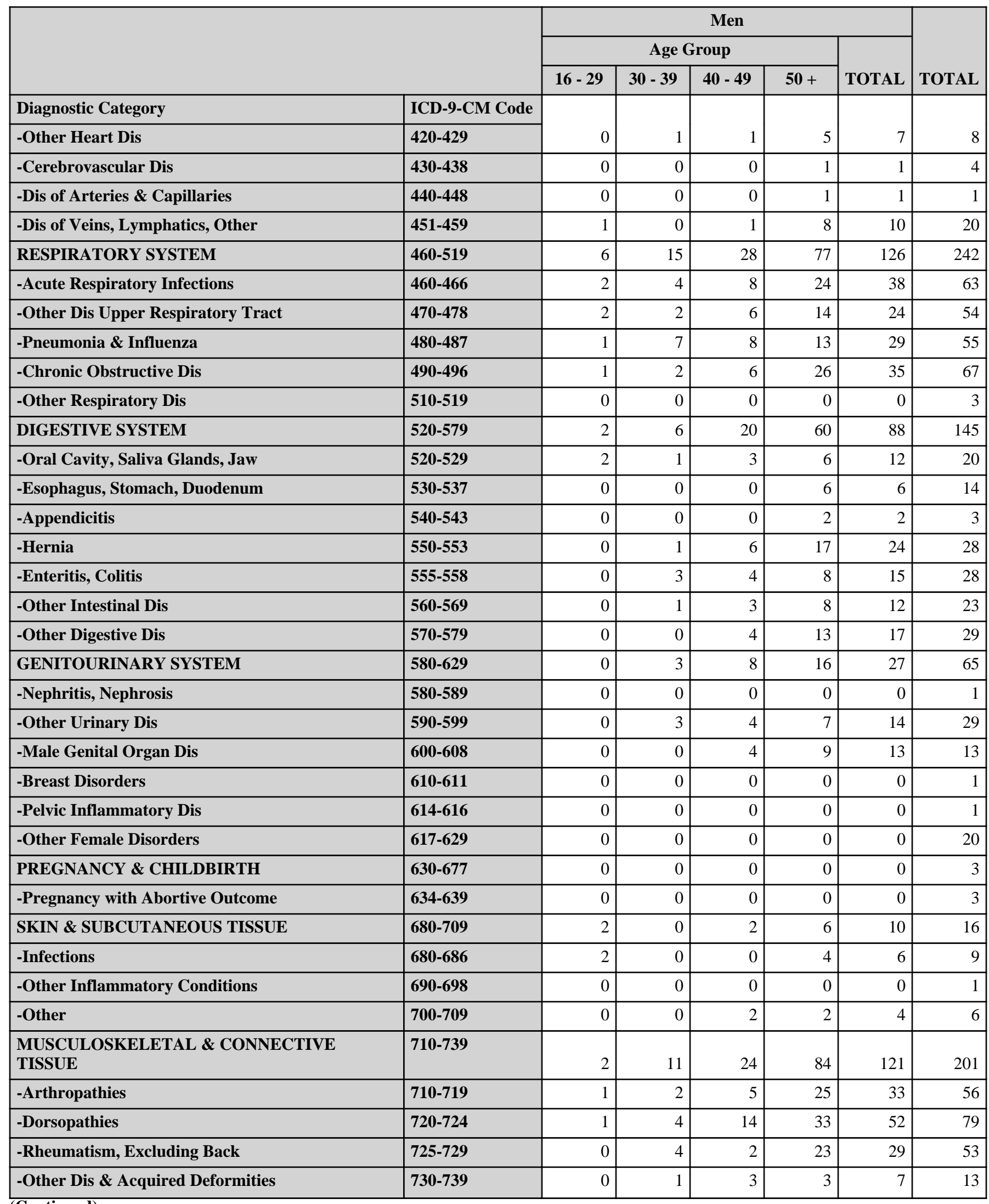

(Continued)

*Only those diagnostic categories and gender/age combinations with at least one occurrence appear in this table. 
Oak Ridge National Laboratory 2008

Absence Data

Appendix G. Number of Diagnoses in Each Diagnostic Category by Gender and Age*

\begin{tabular}{|c|c|c|c|c|c|c|}
\hline & & \multicolumn{5}{|c|}{ Women } \\
\hline & & \multicolumn{4}{|c|}{ Age Group } & \multirow[b]{2}{*}{ TOTAL } \\
\hline & & $16-29$ & $30-39$ & $40-49$ & $50+$ & \\
\hline Diagnostic Category & ICD-9-CM Code & \multirow[b]{2}{*}{0} & \multirow[b]{2}{*}{0} & \multirow[b]{2}{*}{1} & \multirow[b]{2}{*}{1} & \multirow[b]{2}{*}{2} \\
\hline CONGENITAL ANOMALIES & 740-759 & & & & & \\
\hline $\begin{array}{l}\text { SYMPTOMS, SIGNS, \& ILL-DEFINED } \\
\text { CONDITIONS }\end{array}$ & $780-799$ & 1 & 5 & 11 & 21 & 38 \\
\hline -Symptoms & 780-789 & 1 & 5 & 11 & 19 & 36 \\
\hline -Non-Specific Abnormal Findings & $790-796$ & 0 & 0 & 0 & 2 & 2 \\
\hline INJURY \& POISONING & 800-999 & 2 & 3 & 25 & 31 & 61 \\
\hline -Fracture - Skull & 800-804 & 0 & 0 & 0 & 1 & 1 \\
\hline -Fracture - Neck, Trunk & 805-809 & 0 & 0 & 1 & 2 & 3 \\
\hline -Fracture - Upper Limb & 810-819 & 0 & 0 & 1 & 0 & 1 \\
\hline -Fracture - Lower Limb & $820-829$ & 0 & 0 & 3 & 2 & 5 \\
\hline -Dislocation & $830-839$ & 0 & 0 & 2 & 10 & 12 \\
\hline -Sprains \& Strains - Back & 846-847 & 2 & 0 & 0 & 1 & 3 \\
\hline -Sprains \& Strains - Other & $840-845,848$ & 0 & 1 & 5 & 4 & 10 \\
\hline -Intracranial Injury & $850-854$ & 0 & 0 & 1 & 0 & 1 \\
\hline -Internal Injury - Thorax, Abdomen, Pelvis & $860-869$ & 0 & 0 & 0 & 1 & 1 \\
\hline -Open Wound - Head, Neck, Trunk & 870-879 & 0 & 0 & 0 & 3 & 3 \\
\hline -Open Wound - Upper Limb & 880-887 & 0 & 0 & 2 & 0 & 2 \\
\hline -Open Wound - Lower Limb & 890-897 & 0 & 0 & 1 & 0 & 1 \\
\hline -Late Effects of Accident & 905-909 & 0 & 0 & 0 & 1 & 1 \\
\hline -Superficial Injury & 910-919 & 0 & 0 & 1 & 0 & 1 \\
\hline -Contusion & $920-924$ & 0 & 0 & 6 & 1 & 7 \\
\hline -Foreign Body Entering Orifice & 930-939 & 0 & 0 & 0 & 0 & 0 \\
\hline -Burns & $940-949$ & 0 & 0 & 0 & 0 & 0 \\
\hline -Complications \& Unspecified Injuries & 958-959 & 0 & 1 & 0 & 0 & 1 \\
\hline -Poisoning - Medicinal/Biological & $960-979$ & 0 & 0 & 1 & 0 & 1 \\
\hline -Unspecified Effects - External Causes & $990-995$ & 0 & 0 & 0 & 0 & 0 \\
\hline -Complications of Surgical/Medical Care & 996-999 & 0 & 1 & 1 & 5 & 7 \\
\hline $\begin{array}{l}\text { HEALTH STATUS/HEALTH SERVICE } \\
\text { CONTACT }\end{array}$ & V01-V82 & 0 & 1 & 2 & 2 & 5 \\
\hline -Isolation \& Prophylactic Measures & V07-V09 & 0 & 0 & 0 & 0 & 0 \\
\hline -Personal \& Family History & V10-V19 & 0 & 0 & 0 & 0 & 0 \\
\hline -Health Services Reproduction/Development & V20-V29 & 0 & 0 & 1 & 0 & 1 \\
\hline -Specific Procedures/Aftercare & V50-V59 & 0 & 1 & 1 & 2 & 4 \\
\hline -Examination \& Investigation & V70-V82 & 0 & 0 & 0 & 0 & 0 \\
\hline
\end{tabular}

*Only those diagnostic categories and gender/age combinations with at least one occurrence appear in this table. 
Oak Ridge National Laboratory 2008

Absence Data

Appendix G. Number of Diagnoses in Each Diagnostic Category by Gender and Age*

\begin{tabular}{|c|c|c|c|c|c|c|c|}
\hline & & \multicolumn{5}{|c|}{ Men } & \multirow[b]{3}{*}{ TOTAL } \\
\hline & & \multicolumn{4}{|c|}{ Age Group } & \multirow[b]{2}{*}{ TOTAL } & \\
\hline & & $16-29$ & $30-39$ & $40-49$ & $50+$ & & \\
\hline CONGENITAL ANOMALIES & 740-759 & 0 & 0 & 0 & 1 & 1 & 3 \\
\hline $\begin{array}{l}\text { SYMPTOMS, SIGNS, \& ILL-DEFINED } \\
\text { CONDITIONS }\end{array}$ & $780-799$ & 3 & 6 & 6 & 33 & 48 & 86 \\
\hline -Symptoms & 780-789 & 3 & 6 & 3 & 26 & 38 & 74 \\
\hline -Non-Specific Abnormal Findings & $790-796$ & 0 & 0 & 3 & 7 & 10 & 12 \\
\hline INJURY \& POISONING & $800-999$ & 2 & 24 & 47 & 57 & 130 & 191 \\
\hline -Fracture - Skull & 800-804 & 1 & 0 & 0 & 0 & 1 & 2 \\
\hline -Dislocation & $830-839$ & 0 & 5 & 13 & 16 & 34 & 46 \\
\hline -Sprains \& Strains - Back & 846-847 & 0 & 1 & 3 & 6 & 10 & 13 \\
\hline -Sprains \& Strains - Other & $840-845,848$ & 0 & 8 & 12 & 16 & 36 & 46 \\
\hline -Intracranial Injury & $850-854$ & 0 & 0 & 0 & 0 & 0 & 1 \\
\hline -Internal Injury - Thorax, Abdomen, Pelvis & $860-869$ & 0 & 3 & 0 & 1 & 4 & 5 \\
\hline -Open Wound - Head, Neck, Trunk & 870-879 & 0 & 0 & 0 & 0 & 0 & 3 \\
\hline -Open Wound - Upper Limb & 880-887 & 0 & 0 & 1 & 3 & 4 & 6 \\
\hline -Open Wound - Lower Limb & 890-897 & 0 & 0 & 1 & 1 & 2 & 3 \\
\hline -Complications \& Unspecified Injuries & 958-959 & 0 & 1 & 1 & 1 & 3 & 4 \\
\hline -Poisoning - Medicinal/Biological & $960-979$ & 0 & 0 & 0 & 0 & 0 & 1 \\
\hline -Unspecified Effects - External Causes & $990-995$ & 0 & 0 & 0 & 1 & 1 & 1 \\
\hline -Complications of Surgical/Medical Care & 996-999 & 0 & 1 & 1 & 4 & 6 & 13 \\
\hline $\begin{array}{l}\text { HEALTH STATUS/HEALTH SERVICE } \\
\text { CONTACT }\end{array}$ & V01-V82 & 0 & 4 & 1 & 5 & 10 & 15 \\
\hline -Isolation \& Prophylactic Measures & V07-V09 & 0 & 0 & 0 & 1 & 1 & 1 \\
\hline -Personal \& Family History & V10-V19 & 0 & 0 & 0 & 2 & 2 & 2 \\
\hline -Health Services Reproduction/Development & V20-V29 & 0 & 3 & 1 & 0 & 4 & 5 \\
\hline -Specific Procedures/Aftercare & V50-V59 & 0 & 1 & 0 & 1 & 2 & 6 \\
\hline -Examination \& Investigation & V70-V82 & 0 & 0 & 0 & 1 & 1 & 1 \\
\hline
\end{tabular}

*Only those diagnostic categories and gender/age combinations with at least one occurrence appear in this table. 
Oak Ridge National Laboratory 2008

Absence Data

Appendix G. Number of Diagnoses in Each Diagnostic Category by Gender and Age*

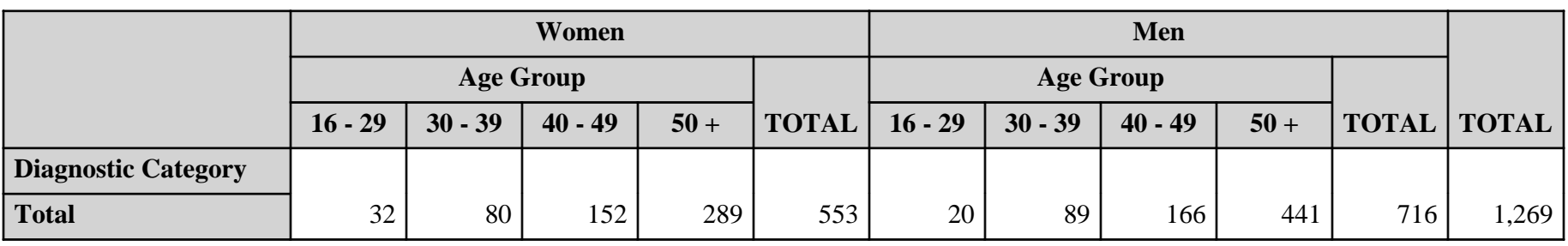

*Only those diagnostic categories and gender/age combinations with at least one occurrence appear in this table. 
Oak Ridge National Laboratory 2008

Absence Data

Appendix H. Total Number of Calendar Days Absent in Each Diagnostic Category by Gender and Age*

\begin{tabular}{|c|c|c|c|c|c|c|}
\hline & & \multicolumn{5}{|c|}{ Women } \\
\hline & & \multicolumn{4}{|c|}{ Age Group } & \multirow[b]{2}{*}{ TOTAL } \\
\hline & & $16-29$ & $30-39$ & $40-49$ & $50+$ & \\
\hline Diagnostic Category & ICD-9-CM Code & \multirow[b]{2}{*}{0} & \multirow[b]{2}{*}{145} & \multirow[b]{2}{*}{22} & \multirow[b]{2}{*}{223} & \multirow[b]{2}{*}{390} \\
\hline INFECTIOUS \& PARASITIC DISEASES (DIS) & 001-139 & & & & & \\
\hline MALIGNANT NEOPLASMS & 140-208, 230-234 & 0 & 164 & 24 & 706 & 894 \\
\hline BENIGN \& UNCERTAIN NEOPLASMS & 210-229, 235-239 & 0 & 41 & 229 & 171 & 441 \\
\hline ENDOCRINE/METABOLIC/IMMUNITY & $240-279$ & 0 & 113 & 97 & 253 & 463 \\
\hline BLOOD \& BLOOD-FORMING ORGANS & $280-289$ & 0 & 70 & 0 & 14 & 84 \\
\hline MENTAL DISORDERS & $290-319$ & 0 & 106 & 343 & 30 & 479 \\
\hline NERVOUS SYSTEM (NS) \& SENSE ORGANS & $320-389$ & 30 & 41 & 52 & 650 & 773 \\
\hline CIRCULATORY SYSTEM & $390-459$ & 0 & 2 & 129 & 162 & 293 \\
\hline RESPIRATORY SYSTEM & $460-519$ & 45 & 102 & 150 & 582 & 879 \\
\hline DIGESTIVE SYSTEM & $520-579$ & 23 & 202 & 185 & 309 & 719 \\
\hline GENITOURINARY SYSTEM & $580-629$ & 16 & 5 & 161 & 339 & 521 \\
\hline PREGNANCY \& CHILDBIRTH & $630-677$ & 19 & 21 & 0 & 0 & 40 \\
\hline SKIN \& SUBCUTANEOUS TISSUE & 680-709 & 0 & 0 & 12 & 29 & 41 \\
\hline $\begin{array}{l}\text { MUSCULOSKELETAL \& CONNECTIVE } \\
\text { TISSUE }\end{array}$ & $710-739$ & 8 & 120 & 610 & 1,571 & 2,309 \\
\hline CONGENITAL ANOMALIES & $740-759$ & 0 & 0 & 64 & 13 & 77 \\
\hline $\begin{array}{l}\text { SYMPTOMS, SIGNS, \& ILL-DEFINED } \\
\text { CONDITIONS }\end{array}$ & $780-799$ & 5 & 165 & 208 & 299 & 677 \\
\hline INJURY \& POISONING & \begin{tabular}{|l|}
$800-999$ \\
\end{tabular} & 9 & 77 & 646 & 984 & 1,716 \\
\hline
\end{tabular}

\footnotetext{
*Absences with >1 ICD-9-CM code in the same diagnostic category were counted only once. Only those diagnostic categories and gender/age combinations with at least one occurrence appear in this table.
} 
Oak Ridge National Laboratory 2008

Absence Data

Appendix H. Total Number of Calendar Days Absent in Each Diagnostic Category by Gender and Age*

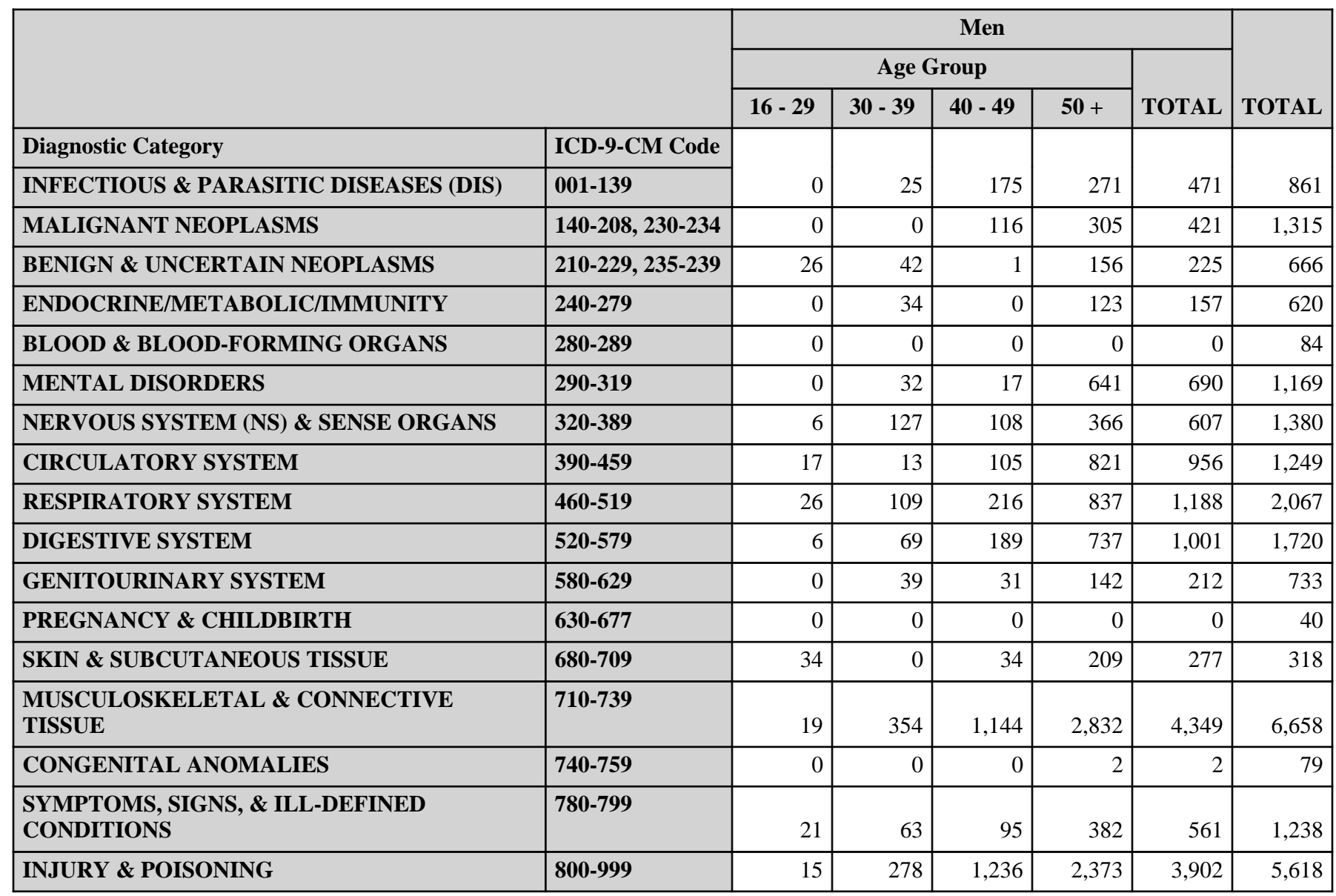

\footnotetext{
*Absences with >1 ICD-9-CM code in the same diagnostic category were counted only once. Only those diagnostic categories and gender/age combinations with at least one occurrence appear in this table.
} 
Oak Ridge National Laboratory 2008

Absence Data

Appendix I. Number of Diagnoses in Each Diagnostic Category by Gender and Job Category*

\begin{tabular}{|c|c|c|c|c|}
\hline & \multicolumn{3}{|c|}{ Women } \\
\hline & & \multicolumn{3}{|c|}{ Job Category } \\
\hline & & Professional & $\begin{array}{c}\text { Administrative } \\
\text { Support }\end{array}$ & Technical Support \\
\hline Diagnostic Category & ICD-9-CM Code & \multirow[b]{2}{*}{9} & \multirow[b]{2}{*}{15} & \multirow[b]{2}{*}{1} \\
\hline INFECTIOUS \& PARASITIC DISEASES (DIS) & 001-139 & & & \\
\hline -Intestinal Infectious Dis & \begin{tabular}{|c|}
$001-009$ \\
\end{tabular} & 1 & 1 & $\overline{0}$ \\
\hline -Other Bacterial Dis & $030-041$ & 2 & 7 & 0 \\
\hline -Viral Dis with Exanthem & 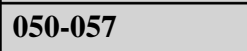 & 0 & 2 & 1 \\
\hline -Other Viral Dis \& Chlamydiae & 070-079 & 6 & 4 & 0 \\
\hline -Mycoses & $110-118$ & 0 & 1 & 0 \\
\hline MALIGNANT NEOPLASMS & $140-208,230-234$ & 5 & 10 & 2 \\
\hline -Respiratory \& Intrathoracic & $160-165$ & 0 & 1 & 0 \\
\hline -Bone, Connective Tissue, Skin & 170-173, 176 & 1 & 0 & 0 \\
\hline -Breast & $174-175$ & 2 & 7 & 0 \\
\hline -Genitourinary & 179-189 & 1 & 0 & 0 \\
\hline -Other \& Unspecified Sites & 190, 193-199 & 1 & 2 & 0 \\
\hline -Carcinoma in situ & $230-234$ & 0 & 0 & 2 \\
\hline BENIGN \& UNCERTAIN NEOPLASMS & 210-229, 235-239 & 11 & 2 & $\overline{0}$ \\
\hline ENDOCRINE/METABOLIC/IMMUNITY & $240-279$ & 2 & 9 & 0 \\
\hline -Thyroid Gland Disorders & $240-246$ & 1 & 3 & 0 \\
\hline -Other Endocrine Gland Dis & $250-259$ & 1 & 1 & 0 \\
\hline -Other Metabolic \& Immunity Disorders & $270-279$ & 0 & 5 & 0 \\
\hline BLOOD \& BLOOD-FORMING ORGANS & $280-289$ & 0 & 1 & 0 \\
\hline MENTAL DISORDERS & $290-319$ & 1 & 2 & 1 \\
\hline -Non-Psychotic Disorders & $300-302,306-316$ & 1 & 2 & 1 \\
\hline NERVOUS SYSTEM (NS) \& SENSE ORGANS & $320-389$ & 17 & 14 & 2 \\
\hline -Inflammatory Dis of Central NS & $320-326$ & 1 & 0 & 0 \\
\hline -Hereditary/Degenerative Central NS Dis & 330-337 & 0 & 0 & 0 \\
\hline -Other Disorders of Central NS & $340-349$ & 2 & 5 & 0 \\
\hline -Disorders of Peripheral NS & $350-359$ & 12 & 2 & 1 \\
\hline -Disorders of Eye & $360-379$ & 0 & 6 & 1 \\
\hline -Dis of Ear \& Mastoid & $380-389$ & 2 & 1 & 0 \\
\hline CIRCULATORY SYSTEM & $390-459$ & 3 & 13 & 2 \\
\hline -Hypertensive Dis & $401-405$ & 0 & 1 & 0 \\
\hline -Ischemic Heart Dis & $410-414$ & 0 & 3 & 0 \\
\hline -Other Heart Dis & $420-429$ & 0 & 1 & 0 \\
\hline -Cerebrovascular Dis & $430-438$ & 1 & 2 & 0 \\
\hline -Dis of Veins, Lymphatics, Other & $451-459$ & 2 & 6 & 2 \\
\hline RESPIRATORY SYSTEM & $460-519$ & 47 & 50 & 14 \\
\hline -Acute Respiratory Infections & $460-466$ & 7 & 13 & 4 \\
\hline
\end{tabular}

(Continued)

*Only those diagnostic categories and gender/job category combinations with at least one occurrence appear in this table. 
Oak Ridge National Laboratory 2008

Absence Data

Appendix I. Number of Diagnoses in Each Diagnostic Category by Gender and Job Category*

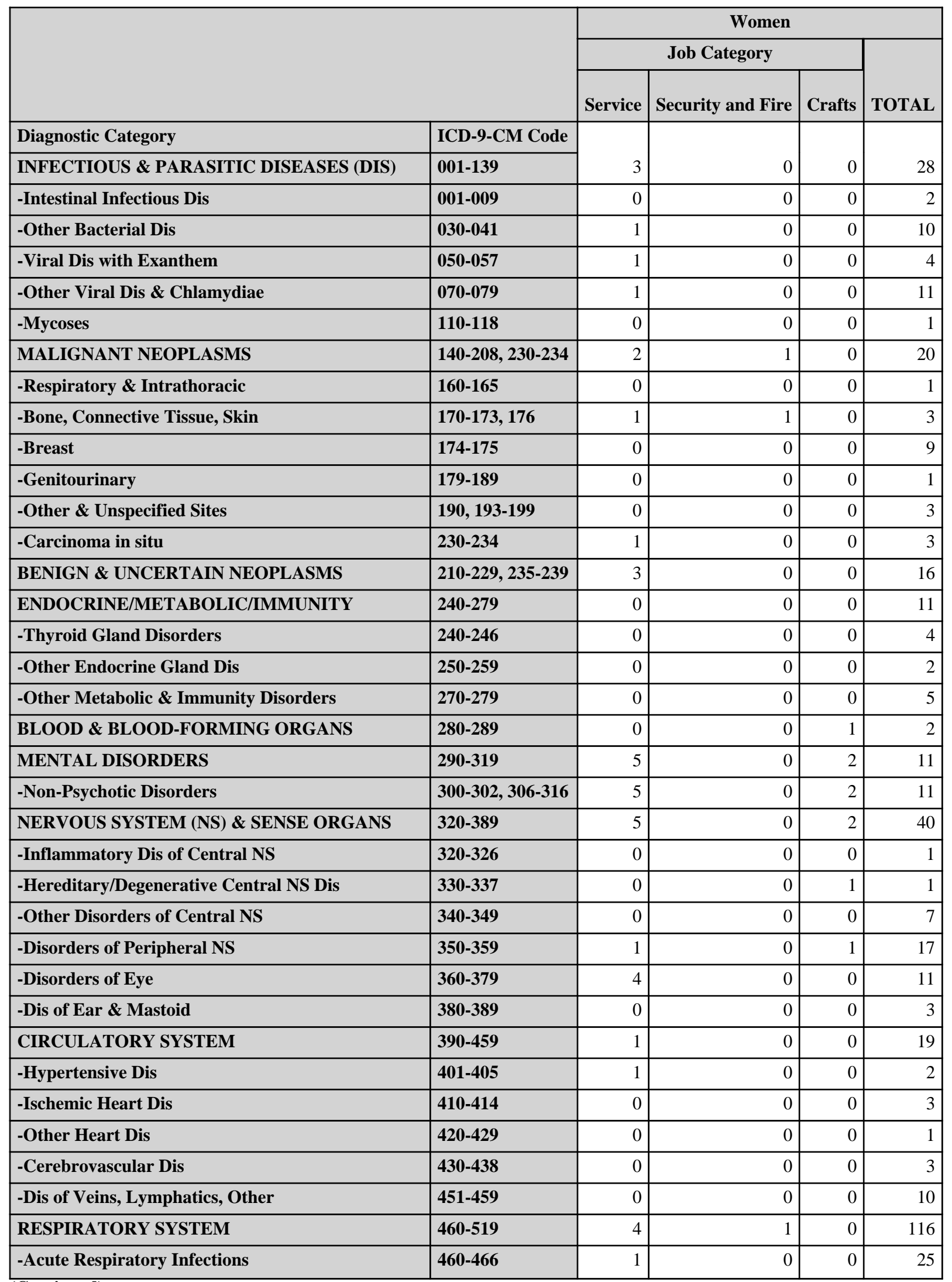

(Continued)

*Only those diagnostic categories and gender/job category combinations with at least one occurrence appear in this table. 
Oak Ridge National Laboratory 2008

Absence Data

Appendix I. Number of Diagnoses in Each Diagnostic Category by Gender and Job Category*

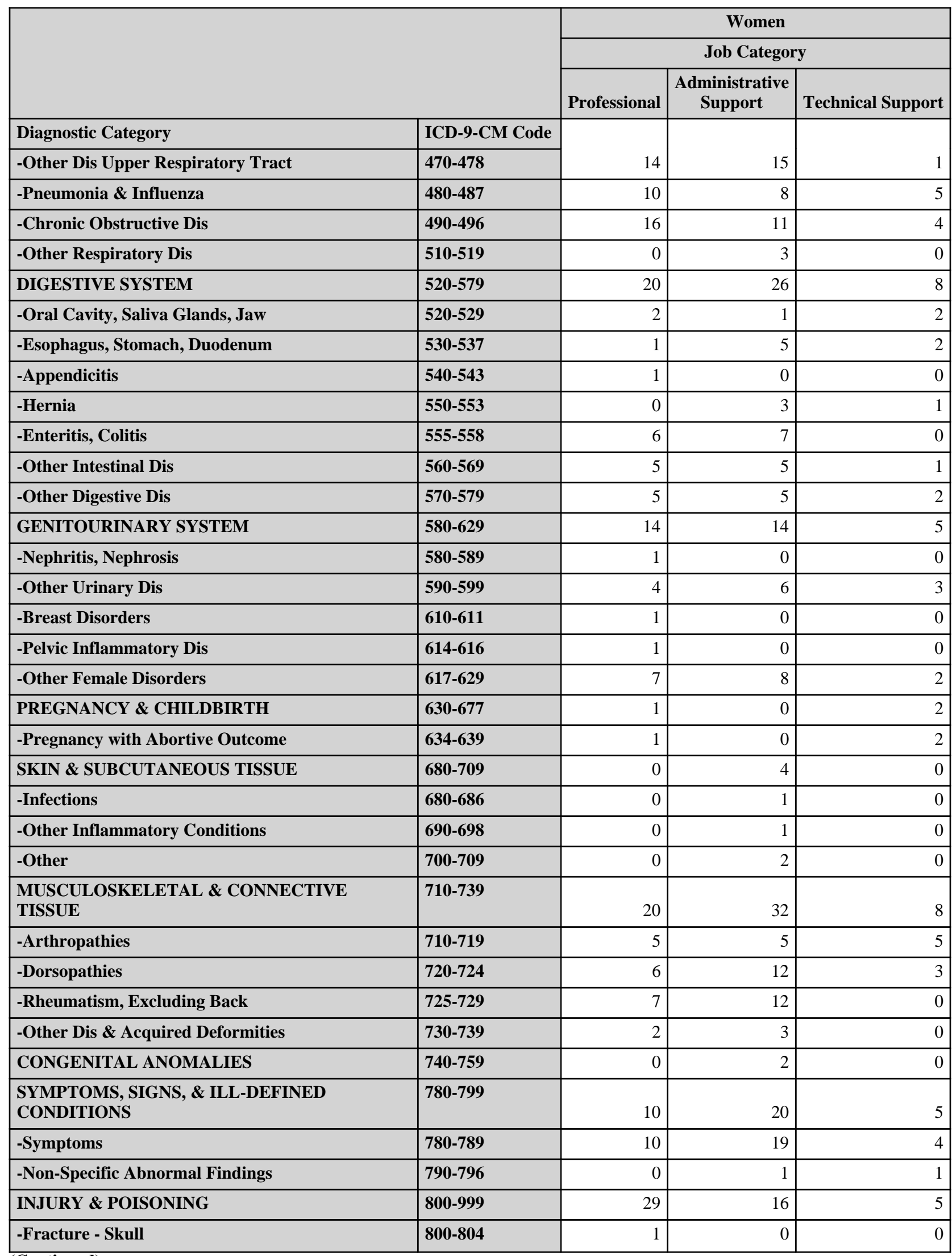

(Continued)

*Only those diagnostic categories and gender/job category combinations with at least one occurrence appear in this table. 
Oak Ridge National Laboratory 2008

Absence Data

Appendix I. Number of Diagnoses in Each Diagnostic Category by Gender and Job Category*

\begin{tabular}{|c|c|c|c|c|c|}
\hline & & \multicolumn{4}{|c|}{ Women } \\
\hline & & \multicolumn{3}{|c|}{ Job Category } & \multirow[b]{2}{*}{ TOTAL } \\
\hline & & Service & Security and Fire & Crafts & \\
\hline Diagnostic Category & ICD-9-CM Code & \multirow[b]{2}{*}{0} & \multirow[b]{2}{*}{0} & \multirow[b]{2}{*}{0} & \multirow[b]{2}{*}{30} \\
\hline -Other Dis Upper Respiratory Tract & $470-478$ & & & & \\
\hline -Pneumonia \& Influenza & $480-487$ & 2 & 1 & 0 & 26 \\
\hline -Chronic Obstructive Dis & $490-496$ & 1 & 0 & 0 & 32 \\
\hline -Other Respiratory Dis & $510-519$ & 0 & 0 & 0 & 3 \\
\hline DIGESTIVE SYSTEM & $520-579$ & 3 & 0 & 0 & 57 \\
\hline -Oral Cavity, Saliva Glands, Jaw & $520-529$ & 3 & 0 & 0 & 8 \\
\hline -Esophagus, Stomach, Duodenum & $\mathbf{5 3 0 - 5 3 7}$ & 0 & 0 & 0 & 8 \\
\hline -Appendicitis & $540-543$ & 0 & 0 & 0 & 1 \\
\hline -Hernia & $550-553$ & 0 & 0 & 0 & 4 \\
\hline -Enteritis, Colitis & $555-558$ & 0 & 0 & 0 & 13 \\
\hline -Other Intestinal Dis & $560-569$ & 0 & 0 & 0 & 11 \\
\hline -Other Digestive Dis & $570-579$ & 0 & 0 & 0 & 12 \\
\hline GENITOURINARY SYSTEM & $580-629$ & 5 & 0 & 0 & 38 \\
\hline -Nephritis, Nephrosis & $580-589$ & 0 & 0 & 0 & 1 \\
\hline -Other Urinary Dis & $590-599$ & 2 & 0 & 0 & 15 \\
\hline -Breast Disorders & $610-611$ & 0 & 0 & 0 & 1 \\
\hline -Pelvic Inflammatory Dis & $614-616$ & 0 & 0 & 0 & 1 \\
\hline -Other Female Disorders & $617-629$ & 3 & 0 & 0 & 20 \\
\hline PREGNANCY \& CHILDBIRTH & $630-677$ & 0 & 0 & 0 & 3 \\
\hline -Pregnancy with Abortive Outcome & 634-639 & 0 & 0 & 0 & 3 \\
\hline SKIN \& SUBCUTANEOUS TISSUE & $680-709$ & 2 & 0 & 0 & 6 \\
\hline -Infections & $680-686$ & 2 & 0 & 0 & 3 \\
\hline -Other Inflammatory Conditions & 690-698 & 0 & 0 & 0 & 1 \\
\hline -Other & 700-709 & 0 & 0 & 0 & 2 \\
\hline $\begin{array}{l}\text { MUSCULOSKELETAL \& CONNECTIVE } \\
\text { TISSUE }\end{array}$ & $710-739$ & 16 & 0 & 4 & 80 \\
\hline -Arthropathies & $710-719$ & 6 & 0 & 2 & 23 \\
\hline -Dorsopathies & $720-724$ & 4 & 0 & 2 & 27 \\
\hline -Rheumatism, Excluding Back & $725-729$ & 5 & 0 & 0 & 24 \\
\hline -Other Dis \& Acquired Deformities & 730-739 & 1 & 0 & 0 & 6 \\
\hline CONGENITAL ANOMALIES & $740-759$ & 0 & 0 & 0 & 2 \\
\hline $\begin{array}{l}\text { SYMPTOMS, SIGNS, \& ILL-DEFINED } \\
\text { CONDITIONS }\end{array}$ & 780-799 & 2 & 0 & 1 & 38 \\
\hline -Symptoms & 780-789 & 2 & 0 & 1 & 36 \\
\hline -Non-Specific Abnormal Findings & $790-796$ & 0 & 0 & 0 & 2 \\
\hline INJURY \& POISONING & $800-999$ & 10 & 1 & 0 & 61 \\
\hline -Fracture - Skull & $800-804$ & 0 & 0 & 0 & 1 \\
\hline
\end{tabular}

(Continued)

*Only those diagnostic categories and gender/job category combinations with at least one occurrence appear in this table. 
Oak Ridge National Laboratory 2008

Absence Data

Appendix I. Number of Diagnoses in Each Diagnostic Category by Gender and Job Category*

\begin{tabular}{|c|c|c|c|c|}
\hline & & \multicolumn{3}{|c|}{ Women } \\
\hline & & \multicolumn{3}{|c|}{ Job Category } \\
\hline & & Professional & $\begin{array}{l}\text { Administrative } \\
\text { Support }\end{array}$ & Technical Support \\
\hline Diagnostic Category & ICD-9-CM Code & \multirow[b]{2}{*}{1} & \multirow[b]{2}{*}{0} & \multirow[b]{2}{*}{0} \\
\hline -Fracture - Neck, Trunk & 805-809 & & & \\
\hline -Fracture - Upper Limb & $810-819$ & 0 & 0 & 0 \\
\hline -Fracture - Lower Limb & 820-829 & 1 & 1 & 1 \\
\hline -Dislocation & 830-839 & 6 & 5 & 0 \\
\hline -Sprains \& Strains - Back & 846-847 & 2 & 1 & 0 \\
\hline -Sprains \& Strains - Other & $840-845,848$ & 6 & 2 & 1 \\
\hline -Intracranial Injury & $850-854$ & 1 & 0 & 0 \\
\hline -Internal Injury - Thorax, Abdomen, Pelvis & $860-869$ & 0 & 0 & 1 \\
\hline -Open Wound - Head, Neck, Trunk & 870-879 & 3 & 0 & 0 \\
\hline -Open Wound - Upper Limb & $880-887$ & 0 & 0 & 1 \\
\hline -Open Wound - Lower Limb & $890-897$ & 0 & 0 & 0 \\
\hline -Late Effects of Accident & 905-909 & 0 & 1 & 0 \\
\hline -Superficial Injury & $910-919$ & 1 & 0 & 0 \\
\hline -Contusion & $920-924$ & 4 & 2 & 0 \\
\hline -Complications \& Unspecified Injuries & 958-959 & 0 & 0 & 1 \\
\hline -Poisoning - Medicinal/Biological & $960-979$ & 0 & 0 & 0 \\
\hline -Complications of Surgical/Medical Care & 996-999 & 3 & 4 & 0 \\
\hline $\begin{array}{l}\text { HEALTH STATUS/HEALTH SERVICE } \\
\text { CONTACT }\end{array}$ & V01-V82 & 2 & 2 & 0 \\
\hline -Health Services Reproduction/Development & V20-V29 & 1 & 0 & 0 \\
\hline -Specific Procedures/Aftercare & V50-V59 & 1 & 2 & 0 \\
\hline
\end{tabular}

*Only those diagnostic categories and gender/job category combinations with at least one occurrence appear in this table. 
Oak Ridge National Laboratory 2008

Absence Data

Appendix I. Number of Diagnoses in Each Diagnostic Category by Gender and Job Category*

\begin{tabular}{|c|c|c|c|c|c|}
\hline & & \multicolumn{4}{|c|}{ Women } \\
\hline & & \multicolumn{3}{|c|}{ Job Category } & \multirow[b]{2}{*}{ TOTAL } \\
\hline & & Service & Security and Fire & Crafts & \\
\hline Diagnostic Category & ICD-9-CM Code & \multirow[b]{2}{*}{1} & \multirow[b]{2}{*}{1} & \multirow[b]{2}{*}{0} & \multirow[b]{2}{*}{3} \\
\hline -Fracture - Neck, Trunk & 805-809 & & & & \\
\hline -Fracture - Upper Limb & $810-819$ & 1 & 0 & 0 & 1 \\
\hline -Fracture - Lower Limb & 820-829 & 2 & 0 & 0 & 5 \\
\hline -Dislocation & $830-839$ & 1 & 0 & 0 & 12 \\
\hline -Sprains \& Strains - Back & 846-847 & 0 & 0 & 0 & 3 \\
\hline -Sprains \& Strains - Other & $840-845,848$ & 1 & 0 & 0 & 10 \\
\hline -Intracranial Injury & $850-854$ & 0 & 0 & 0 & 1 \\
\hline -Internal Injury - Thorax, Abdomen, Pelvis & $860-869$ & 0 & 0 & 0 & 1 \\
\hline -Open Wound - Head, Neck, Trunk & 870-879 & 0 & 0 & 0 & 3 \\
\hline -Open Wound - Upper Limb & 880-887 & 1 & 0 & 0 & 2 \\
\hline -Open Wound - Lower Limb & $890-897$ & 1 & 0 & 0 & 1 \\
\hline -Late Effects of Accident & 905-909 & 0 & 0 & 0 & 1 \\
\hline -Superficial Injury & $910-919$ & 0 & 0 & 0 & 1 \\
\hline -Contusion & $920-924$ & 1 & 0 & 0 & 7 \\
\hline -Complications \& Unspecified Injuries & \begin{tabular}{|c|}
$958-959$ \\
\end{tabular} & 0 & 0 & 0 & 1 \\
\hline -Poisoning - Medicinal/Biological & $960-979$ & 1 & 0 & 0 & 1 \\
\hline -Complications of Surgical/Medical Care & 996-999 & 0 & 0 & 0 & 7 \\
\hline $\begin{array}{l}\text { HEALTH STATUS/HEALTH SERVICE } \\
\text { CONTACT }\end{array}$ & V01-V82 & 1 & 0 & 0 & 5 \\
\hline -Health Services Reproduction/Development & V20-V29 & 0 & 0 & 0 & 1 \\
\hline -Specific Procedures/Aftercare & V50-V59 & 1 & 0 & 0 & 4 \\
\hline
\end{tabular}

\begin{tabular}{|c|c|c|c|c|c|c|c|}
\hline & \multicolumn{7}{|c|}{ Women } \\
\hline & \multicolumn{6}{|c|}{ Job Category } & \multirow[b]{2}{*}{ TOTAL } \\
\hline & Professional & $\begin{array}{c}\text { Administrative } \\
\text { Support }\end{array}$ & Technical Support & Service & Security and Fire & Crafts & \\
\hline Diagnostic Category & \multirow[b]{2}{*}{191} & \multirow[b]{2}{*}{232} & \multirow[b]{2}{*}{55} & \multirow[b]{2}{*}{62} & \multirow[b]{2}{*}{3} & \multirow[b]{2}{*}{10} & \multirow[b]{2}{*}{553} \\
\hline Total & & & & & & & \\
\hline
\end{tabular}

*Only those diagnostic categories and gender/job category combinations with at least one occurrence appear in this table. 
Oak Ridge National Laboratory 2008

Absence Data

Appendix I. Number of Diagnoses in Each Diagnostic Category by Gender and Job Category*

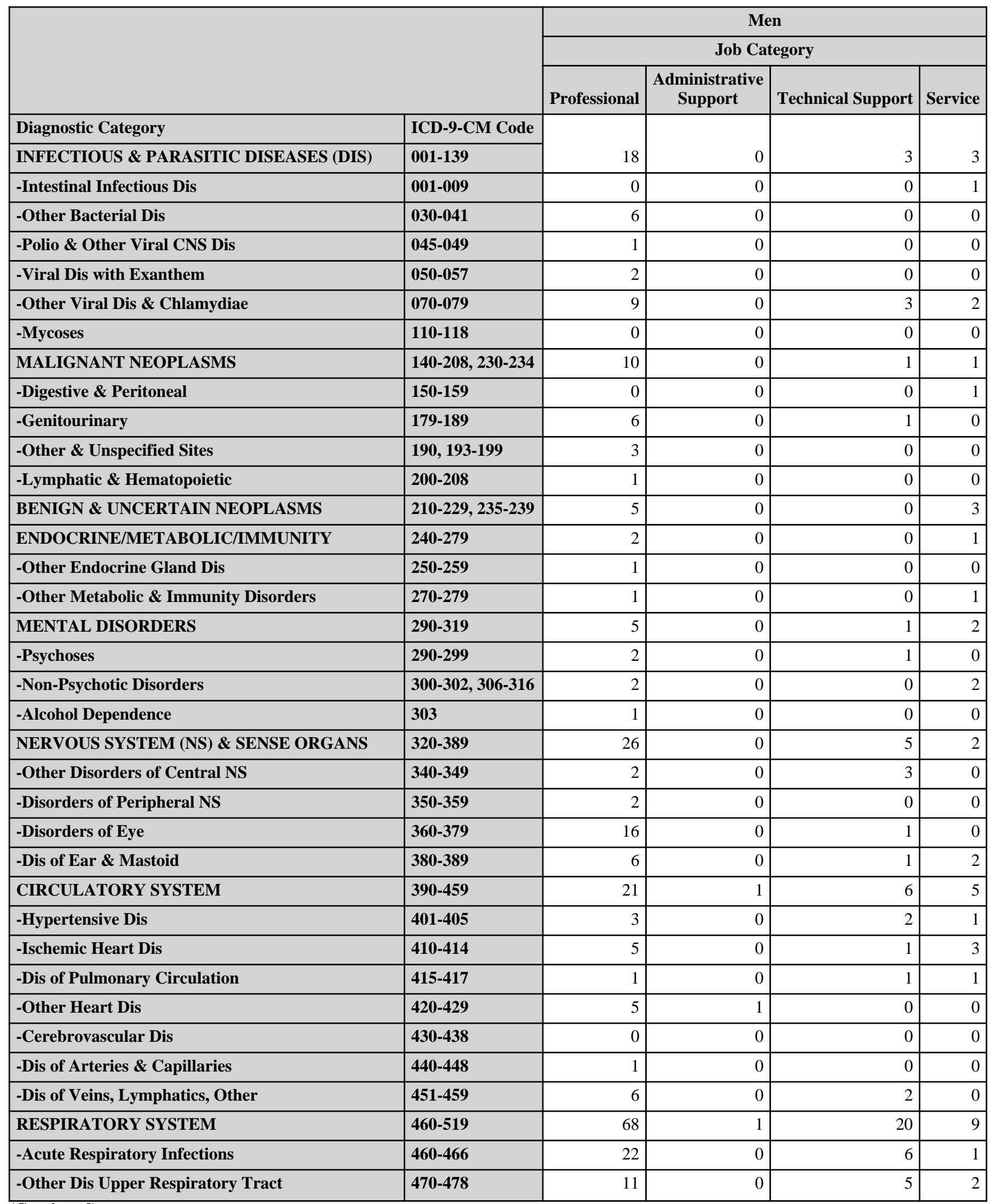

(Continued)

*Only those diagnostic categories and gender/job category combinations with at least one occurrence appear in this table. 
Oak Ridge National Laboratory 2008

Absence Data

Appendix I. Number of Diagnoses in Each Diagnostic Category by Gender and Job Category*

\begin{tabular}{|c|c|c|c|c|c|}
\hline & & \multicolumn{4}{|c|}{ Men } \\
\hline & & \multicolumn{3}{|c|}{ Job Category } & \multirow[b]{2}{*}{ TOTAL } \\
\hline & & Security and Fire & Crafts & Line Operators & \\
\hline Diagnostic Category & ICD-9-CM Code & \multirow[b]{2}{*}{2} & \multirow[b]{2}{*}{8} & \multirow[b]{2}{*}{0} & \multirow[b]{2}{*}{34} \\
\hline INFECTIOUS \& PARASITIC DISEASES (DIS) & 001-139 & & & & \\
\hline -Intestinal Infectious Dis & 001-009 & 0 & 0 & 0 & 1 \\
\hline -Other Bacterial Dis & 030-041 & 0 & 5 & 0 & 11 \\
\hline -Polio \& Other Viral CNS Dis & 045-049 & 0 & 0 & 0 & 1 \\
\hline -Viral Dis with Exanthem & $050-057$ & 0 & 0 & 0 & 2 \\
\hline -Other Viral Dis \& Chlamydiae & 070-079 & 1 & 3 & 0 & 18 \\
\hline -Mycoses & $110-118$ & 1 & 0 & 0 & 1 \\
\hline MALIGNANT NEOPLASMS & $140-208,230-234$ & 0 & 3 & 0 & 15 \\
\hline -Digestive \& Peritoneal & $150-159$ & 0 & 0 & 0 & 1 \\
\hline -Genitourinary & 179-189 & 0 & 3 & 0 & 10 \\
\hline -Other \& Unspecified Sites & $190,193-199$ & 0 & 0 & 0 & 3 \\
\hline -Lymphatic \& Hematopoietic & $200-208$ & 0 & 0 & 0 & 1 \\
\hline BENIGN \& UNCERTAIN NEOPLASMS & $210-229,235-239$ & 0 & 1 & 1 & 10 \\
\hline ENDOCRINE/METABOLIC/IMMUNITY & $240-279$ & 1 & 0 & 0 & 4 \\
\hline -Other Endocrine Gland Dis & $250-259$ & 0 & 0 & 0 & 1 \\
\hline -Other Metabolic \& Immunity Disorders & $270-279$ & 1 & 0 & 0 & 3 \\
\hline MENTAL DISORDERS & $290-319$ & 0 & 3 & 0 & 11 \\
\hline -Psychoses & $290-299$ & 0 & 0 & 0 & 3 \\
\hline -Non-Psychotic Disorders & $300-302,306-316$ & 0 & 3 & 0 & 7 \\
\hline -Alcohol Dependence & 303 & 0 & 0 & 0 & 1 \\
\hline NERVOUS SYSTEM (NS) \& SENSE ORGANS & $320-389$ & 0 & 3 & 0 & 36 \\
\hline -Other Disorders of Central NS & $340-349$ & 0 & 0 & 0 & 5 \\
\hline -Disorders of Peripheral NS & $350-359$ & 0 & 1 & 0 & 3 \\
\hline -Disorders of Eye & $360-379$ & 0 & 0 & 0 & 17 \\
\hline -Dis of Ear \& Mastoid & $380-389$ & 0 & 2 & 0 & 11 \\
\hline CIRCULATORY SYSTEM & $390-459$ & 0 & 11 & 1 & 45 \\
\hline -Hypertensive Dis & $401-405$ & 0 & 1 & 0 & 7 \\
\hline -Ischemic Heart Dis & $410-414$ & 0 & 6 & 0 & 15 \\
\hline -Dis of Pulmonary Circulation & $415-417$ & 0 & 0 & 1 & 4 \\
\hline -Other Heart Dis & $420-429$ & 0 & 1 & 0 & 7 \\
\hline -Cerebrovascular Dis & $430-438$ & 0 & 1 & 0 & 1 \\
\hline -Dis of Arteries \& Capillaries & $440-448$ & 0 & 0 & 0 & 1 \\
\hline -Dis of Veins, Lymphatics, Other & 451-459 & 0 & 2 & 0 & 10 \\
\hline RESPIRATORY SYSTEM & $460-519$ & 3 & 24 & 1 & 126 \\
\hline -Acute Respiratory Infections & $460-466$ & 1 & 7 & 1 & 38 \\
\hline -Other Dis Upper Respiratory Tract & $470-478$ & 1 & 5 & 0 & 24 \\
\hline
\end{tabular}

(Continued)

*Only those diagnostic categories and gender/job category combinations with at least one occurrence appear in this table. 
Oak Ridge National Laboratory 2008

Absence Data

Appendix I. Number of Diagnoses in Each Diagnostic Category by Gender and Job Category*

\begin{tabular}{|c|c|c|c|c|c|}
\hline & \multicolumn{4}{|c|}{ Men } \\
\hline & & \multicolumn{4}{|c|}{ Job Category } \\
\hline & & Professional & $\begin{array}{l}\text { Administrative } \\
\text { Support }\end{array}$ & Technical Support & Service \\
\hline Diagnostic Category & ICD-9-CM Code & \multirow[b]{2}{*}{14} & \multirow[b]{2}{*}{1} & \multirow[b]{2}{*}{3} & \multirow[b]{2}{*}{1} \\
\hline -Pneumonia \& Influenza & $480-487$ & & & & \\
\hline -Chronic Obstructive Dis & $490-496$ & 21 & 0 & 6 & 5 \\
\hline DIGESTIVE SYSTEM & $520-579$ & 47 & 0 & 10 & 9 \\
\hline -Oral Cavity, Saliva Glands, Jaw & $520-529$ & 3 & 0 & 2 & 1 \\
\hline -Esophagus, Stomach, Duodenum & $530-537$ & 2 & 0 & 0 & 2 \\
\hline -Appendicitis & $540-543$ & 2 & 0 & 0 & 0 \\
\hline -Hernia & $550-553$ & 15 & 0 & 1 & 3 \\
\hline -Enteritis, Colitis & $555-558$ & 7 & 0 & 2 & 1 \\
\hline -Other Intestinal Dis & $560-569$ & 8 & 0 & 2 & 2 \\
\hline -Other Digestive Dis & $570-579$ & 10 & 0 & 3 & 0 \\
\hline GENITOURINARY SYSTEM & $580-629$ & 17 & 0 & 1 & 1 \\
\hline -Other Urinary Dis & $590-599$ & 8 & 0 & 1 & 1 \\
\hline -Male Genital Organ Dis & $600-608$ & 9 & 0 & 0 & 0 \\
\hline SKIN \& SUBCUTANEOUS TISSUE & 680-709 & 4 & 0 & 0 & 2 \\
\hline -Infections & $680-686$ & 2 & 0 & 0 & 2 \\
\hline -Other & 700-709 & 2 & 0 & 0 & 0 \\
\hline $\begin{array}{l}\text { MUSCULOSKELETAL \& CONNECTIVE } \\
\text { TISSUE }\end{array}$ & 710-739 & 52 & 0 & 11 & 15 \\
\hline -Arthropathies & 710-719 & 17 & 0 & 1 & 3 \\
\hline -Dorsopathies & 720-724 & 19 & 0 & 8 & 8 \\
\hline -Rheumatism, Excluding Back & $725-729$ & 13 & 0 & 2 & 1 \\
\hline -Other Dis \& Acquired Deformities & 730-739 & 3 & 0 & 0 & 3 \\
\hline CONGENITAL ANOMALIES & 740-759 & 0 & 0 & 0 & 0 \\
\hline $\begin{array}{l}\text { SYMPTOMS, SIGNS, \& ILL-DEFINED } \\
\text { CONDITIONS }\end{array}$ & 780-799 & 21 & 0 & 8 & 9 \\
\hline -Symptoms & 780-789 & 15 & 0 & 8 & 8 \\
\hline -Non-Specific Abnormal Findings & $790-796$ & 6 & 0 & 0 & 1 \\
\hline INJURY \& POISONING & $800-999$ & 60 & 0 & 24 & 4 \\
\hline -Fracture - Skull & $800-804$ & 0 & 0 & 0 & 0 \\
\hline -Fracture - Neck, Trunk & 805-809 & 2 & 0 & 0 & 1 \\
\hline -Fracture - Upper Limb & 810-819 & 3 & 0 & 0 & 1 \\
\hline -Fracture - Lower Limb & $820-829$ & 1 & 0 & 2 & 0 \\
\hline -Dislocation & $830-839$ & 16 & 0 & 5 & 1 \\
\hline -Sprains \& Strains - Back & 846-847 & 5 & 0 & 2 & 0 \\
\hline -Sprains \& Strains - Other & $840-845,848$ & 15 & 0 & 9 & 0 \\
\hline -Internal Injury - Thorax, Abdomen, Pelvis & $860-869$ & 1 & 0 & 3 & 0 \\
\hline -Open Wound - Upper Limb & 880-887 & 1 & 0 & 0 & 0 \\
\hline
\end{tabular}

(Continued)

*Only those diagnostic categories and gender/job category combinations with at least one occurrence appear in this table. 
Oak Ridge National Laboratory 2008

Absence Data

Appendix I. Number of Diagnoses in Each Diagnostic Category by Gender and Job Category*

\begin{tabular}{|c|c|c|c|c|c|}
\hline & & \multicolumn{4}{|c|}{ Men } \\
\hline & & \multicolumn{3}{|c|}{ Job Category } & \multirow[b]{2}{*}{ TOTAL } \\
\hline & & Security and Fire & Crafts & Line Operators & \\
\hline Diagnostic Category & ICD-9-CM Code & \multirow[b]{2}{*}{0} & \multirow[b]{2}{*}{10} & \multirow[b]{2}{*}{0} & \multirow[b]{2}{*}{29} \\
\hline -Pneumonia \& Influenza & $480-487$ & & & & \\
\hline -Chronic Obstructive Dis & $490-496$ & 1 & 2 & 0 & 35 \\
\hline DIGESTIVE SYSTEM & $520-579$ & 2 & 17 & 3 & 88 \\
\hline -Oral Cavity, Saliva Glands, Jaw & $520-529$ & 1 & 5 & 0 & 12 \\
\hline -Esophagus, Stomach, Duodenum & 530-537 & 0 & 2 & 0 & 6 \\
\hline -Appendicitis & $540-543$ & 0 & 0 & 0 & 2 \\
\hline -Hernia & $550-553$ & 1 & 3 & 1 & 24 \\
\hline -Enteritis, Colitis & $555-558$ & 0 & 3 & 2 & 15 \\
\hline -Other Intestinal Dis & $560-569$ & 0 & 0 & 0 & 12 \\
\hline -Other Digestive Dis & $570-579$ & 0 & 4 & 0 & 17 \\
\hline GENITOURINARY SYSTEM & $580-629$ & 0 & 8 & 0 & 27 \\
\hline -Other Urinary Dis & $590-599$ & 0 & 4 & 0 & 14 \\
\hline -Male Genital Organ Dis & $600-608$ & 0 & 4 & 0 & 13 \\
\hline SKIN \& SUBCUTANEOUS TISSUE & 680-709 & 0 & 4 & 0 & 10 \\
\hline -Infections & $680-686$ & 0 & 2 & 0 & 6 \\
\hline -Other & 700-709 & 0 & 2 & 0 & 4 \\
\hline $\begin{array}{l}\text { MUSCULOSKELETAL \& CONNECTIVE } \\
\text { TISSUE }\end{array}$ & 710-739 & 5 & 38 & 0 & 121 \\
\hline -Arthropathies & $710-719$ & 1 & 11 & 0 & 33 \\
\hline -Dorsopathies & $720-724$ & 1 & 16 & 0 & 52 \\
\hline -Rheumatism, Excluding Back & 725-729 & 2 & 11 & 0 & 29 \\
\hline -Other Dis \& Acquired Deformities & 730-739 & 1 & 0 & 0 & 7 \\
\hline CONGENITAL ANOMALIES & $740-759$ & 0 & 1 & 0 & 1 \\
\hline $\begin{array}{l}\text { SYMPTOMS, SIGNS, \& ILL-DEFINED } \\
\text { CONDITIONS }\end{array}$ & $780-799$ & 2 & 8 & 0 & 48 \\
\hline -Symptoms & 780-789 & 2 & 5 & 0 & 38 \\
\hline -Non-Specific Abnormal Findings & $790-796$ & 0 & 3 & 0 & 10 \\
\hline INJURY \& POISONING & $800-999$ & 3 & 37 & 2 & 130 \\
\hline -Fracture - Skull & 800-804 & 0 & 1 & 0 & 1 \\
\hline -Fracture - Neck, Trunk & 805-809 & 0 & 0 & 0 & 3 \\
\hline -Fracture - Upper Limb & 810-819 & 0 & 4 & 0 & 8 \\
\hline -Fracture - Lower Limb & $820-829$ & 0 & 0 & 0 & 3 \\
\hline -Dislocation & 830-839 & 1 & 10 & 1 & 34 \\
\hline -Sprains \& Strains - Back & 846-847 & 0 & 3 & 0 & 10 \\
\hline -Sprains \& Strains - Other & $840-845,848$ & 0 & 11 & 1 & 36 \\
\hline -Internal Injury - Thorax, Abdomen, Pelvis & $860-869$ & 0 & 0 & 0 & 4 \\
\hline -Open Wound - Upper Limb & $880-887$ & 1 & 2 & 0 & 4 \\
\hline
\end{tabular}

(Continued)

*Only those diagnostic categories and gender/job category combinations with at least one occurrence appear in this table. 
Oak Ridge National Laboratory 2008

Absence Data

Appendix I. Number of Diagnoses in Each Diagnostic Category by Gender and Job Category*

\begin{tabular}{|c|c|c|c|c|c|}
\hline & & \multicolumn{4}{|c|}{ Men } \\
\hline & & \multicolumn{4}{|c|}{ Job Category } \\
\hline & & Professional & $\begin{array}{l}\text { Administrative } \\
\text { Support }\end{array}$ & Technical Support & Service \\
\hline Diagnostic Category & ICD-9-CM Code & \multirow[b]{2}{*}{1} & \multirow[b]{2}{*}{0} & \multirow[b]{2}{*}{0} & \multirow[b]{2}{*}{0} \\
\hline -Open Wound - Lower Limb & 890-897 & & & & \\
\hline -Contusion & $920-924$ & 7 & 0 & 0 & 1 \\
\hline -Foreign Body Entering Orifice & $930-939$ & 0 & 0 & 0 & 0 \\
\hline -Burns & 940-949 & 0 & 0 & 2 & 0 \\
\hline -Complications \& Unspecified Injuries & 958-959 & 1 & 0 & 1 & 0 \\
\hline -Isolation \& Prophylactic Measures & V07-V09 & 0 & 0 & 0 & 0 \\
\hline -Personal \& Family History & V10-V19 & 1 & 0 & 0 & 0 \\
\hline -Health Services Reproduction/Development & V20-V29 & 1 & 0 & 0 & 0 \\
\hline -Specific Procedures/Aftercare & V50-V59 & 2 & 0 & 0 & 0 \\
\hline -Examination \& Investigation & V70-V82 & 0 & 0 & 0 & 0 \\
\hline
\end{tabular}

*Only those diagnostic categories and gender/job category combinations with at least one occurrence appear in this table. 
Oak Ridge National Laboratory 2008

Absence Data

Appendix I. Number of Diagnoses in Each Diagnostic Category by Gender and Job Category*

\begin{tabular}{|c|c|c|c|c|c|}
\hline & & \multicolumn{4}{|c|}{ Men } \\
\hline & & \multicolumn{3}{|c|}{ Job Category } & \multirow[b]{2}{*}{ TOTAL } \\
\hline & & Security and Fire & Crafts & Line Operators & \\
\hline Diagnostic Category & ICD-9-CM Code & \multirow[b]{2}{*}{0} & \multirow[b]{2}{*}{1} & \multirow[b]{2}{*}{0} & \multirow[b]{2}{*}{2} \\
\hline -Open Wound - Lower Limb & 890-897 & & & & \\
\hline -Superficial Injury & 910-919 & 0 & 0 & 0 & 1 \\
\hline -Contusion & 920-924 & 0 & 3 & 0 & 11 \\
\hline -Foreign Body Entering Orifice & 930-939 & 1 & 0 & 0 & 1 \\
\hline -Burns & 940-949 & 0 & 0 & 0 & 2 \\
\hline -Complications \& Unspecified Injuries & 958-959 & 0 & 1 & 0 & 3 \\
\hline -Unspecified Effects - External Causes & $990-995$ & 0 & 0 & 0 & 1 \\
\hline -Complications of Surgical/Medical Care & 996-999 & 0 & 1 & 0 & 6 \\
\hline $\begin{array}{l}\text { HEALTH STATUS/HEALTH SERVICE } \\
\text { CONTACT }\end{array}$ & V01-V82 & 1 & 5 & 0 & 10 \\
\hline -Isolation \& Prophylactic Measures & V07-V09 & 0 & 1 & 0 & 1 \\
\hline -Personal \& Family History & V10-V19 & 0 & 1 & 0 & 2 \\
\hline -Health Services Reproduction/Development & V20-V29 & 1 & 2 & 0 & 4 \\
\hline -Specific Procedures/Aftercare & V50-V59 & 0 & 0 & 0 & 2 \\
\hline -Examination \& Investigation & V70-V82 & 0 & 1 & 0 & 1 \\
\hline
\end{tabular}

\begin{tabular}{|c|c|c|c|c|c|c|c|c|}
\hline & \multicolumn{8}{|c|}{ Men } \\
\hline & \multicolumn{7}{|c|}{ Job Category } & \multirow[b]{2}{*}{ TOTAL } \\
\hline & Professional & $\begin{array}{c}\text { Administrative } \\
\text { Support }\end{array}$ & \begin{tabular}{c|} 
Technical \\
Support
\end{tabular} & Service & $\begin{array}{l}\text { Security and } \\
\text { Fire }\end{array}$ & Crafts & $\begin{array}{c}\text { Line } \\
\text { Operators }\end{array}$ & \\
\hline Diagnostic Category & \multirow[b]{2}{*}{360} & \multirow[b]{2}{*}{2} & \multirow[b]{2}{*}{90} & \multirow[b]{2}{*}{66} & \multirow[b]{2}{*}{19} & \multirow[b]{2}{*}{171} & \multirow[b]{2}{*}{8} & \multirow[b]{2}{*}{716} \\
\hline Total & & & & & & & & \\
\hline
\end{tabular}

*Only those diagnostic categories and gender/job category combinations with at least one occurrence appear in this table. 
Oak Ridge National Laboratory 2008

Absence Data

Appendix J. Total Number of Calendar Days Absent in Each Diagnostic Category by Gender and Job Category*

\begin{tabular}{|c|c|c|c|c|}
\hline & \multicolumn{3}{|c|}{ Women } \\
\hline & & \multicolumn{3}{|c|}{ Job Category } \\
\hline & & Professional & $\begin{array}{c}\text { Administrative } \\
\text { Support }\end{array}$ & Technical Support \\
\hline Diagnostic Category & ICD-9-CM Code & \multirow[b]{2}{*}{105} & \multirow[b]{2}{*}{223} & \multirow[b]{2}{*}{42} \\
\hline INFECTIOUS \& PARASITIC DISEASES (DIS) & 001-139 & & & \\
\hline MALIGNANT NEOPLASMS & $140-208,230-234$ & 97 & 473 & 61 \\
\hline BENIGN \& UNCERTAIN NEOPLASMS & 210-229, 235-239 & 280 & 69 & 0 \\
\hline ENDOCRINE/METABOLIC/IMMUNITY & $240-279$ & 131 & 332 & 0 \\
\hline BLOOD \& BLOOD-FORMING ORGANS & $280-289$ & 0 & 14 & 0 \\
\hline MENTAL DISORDERS & $290-319$ & 34 & 169 & 14 \\
\hline NERVOUS SYSTEM (NS) \& SENSE ORGANS & 320-389 & 273 & 140 & 12 \\
\hline CIRCULATORY SYSTEM & $390-459$ & 35 & 237 & 20 \\
\hline RESPIRATORY SYSTEM & $460-519$ & 251 & 313 & 84 \\
\hline DIGESTIVE SYSTEM & $520-579$ & 234 & 402 & 73 \\
\hline GENITOURINARY SYSTEM & $580-629$ & 221 & 134 & 58 \\
\hline PREGNANCY \& CHILDBIRTH & $630-677$ & 19 & 0 & 21 \\
\hline SKIN \& SUBCUTANEOUS TISSUE & 680-709 & 0 & 30 & 0 \\
\hline $\begin{array}{l}\text { MUSCULOSKELETAL \& CONNECTIVE } \\
\text { TISSUE }\end{array}$ & 710-739 & 485 & 801 & 83 \\
\hline CONGENITAL ANOMALIES & 740-759 & 0 & 77 & 0 \\
\hline $\begin{array}{l}\text { SYMPTOMS, SIGNS, \& ILL-DEFINED } \\
\text { CONDITIONS }\end{array}$ & 780-799 & 146 & 376 & 11 \\
\hline INJURY \& POISONING & $800-999$ & 607 & 478 & 105 \\
\hline
\end{tabular}

\footnotetext{
*Absences with >1 ICD-9-CM code in the same diagnostic category were counted only once. Only those diagnostic categories and gender/job category combinations with at least one occurrence appear in this table.
} 
Oak Ridge National Laboratory 2008

Absence Data

Appendix J. Total Number of Calendar Days Absent in Each Diagnostic Category by Gender and Job Category*

\begin{tabular}{|c|c|c|c|c|c|}
\hline & & \multicolumn{4}{|c|}{ Women } \\
\hline & & \multicolumn{3}{|c|}{ Job Category } & \multirow[b]{2}{*}{ TOTAI } \\
\hline & & Service & Security and Fire & Crafts & \\
\hline Diagnostic Category & ICD-9-CM Code & \multirow[b]{2}{*}{20} & \multirow[b]{2}{*}{0} & \multirow[b]{2}{*}{0} & \multirow[b]{2}{*}{390} \\
\hline INFECTIOUS \& PARASITIC DISEASES (DIS) & 001-139 & & & & \\
\hline MALIGNANT NEOPLASMS & $140-208,230-234$ & 249 & 14 & 0 & 894 \\
\hline BENIGN \& UNCERTAIN NEOPLASMS & 210-229, 235-239 & 92 & 0 & 0 & 441 \\
\hline ENDOCRINE/METABOLIC/IMMUNITY & 240-279 & 0 & 0 & 0 & 463 \\
\hline BLOOD \& BLOOD-FORMING ORGANS & $280-289$ & 0 & 0 & 70 & 84 \\
\hline MENTAL DISORDERS & 290-319 & 190 & 0 & 72 & 479 \\
\hline NERVOUS SYSTEM (NS) \& SENSE ORGANS & $320-389$ & 127 & 0 & 221 & 773 \\
\hline CIRCULATORY SYSTEM & $390-459$ & 1 & 0 & 0 & 293 \\
\hline RESPIRATORY SYSTEM & $460-519$ & 224 & 7 & 0 & 879 \\
\hline DIGESTIVE SYSTEM & $520-579$ & 10 & 0 & 0 & 719 \\
\hline GENITOURINARY SYSTEM & $580-629$ & 108 & 0 & 0 & 521 \\
\hline PREGNANCY \& CHILDBIRTH & $630-677$ & 0 & 0 & 0 & 40 \\
\hline SKIN \& SUBCUTANEOUS TISSUE & 680-709 & 11 & 0 & 0 & 41 \\
\hline $\begin{array}{l}\text { MUSCULOSKELETAL \& CONNECTIVE } \\
\text { TISSUE }\end{array}$ & $710-739$ & 651 & 0 & 289 & 2,309 \\
\hline CONGENITAL ANOMALIES & $740-759$ & 0 & 0 & 0 & 77 \\
\hline $\begin{array}{l}\text { SYMPTOMS, SIGNS, \& ILL-DEFINED } \\
\text { CONDITIONS }\end{array}$ & 780-799 & 97 & 0 & 47 & 677 \\
\hline INJURY \& POISONING & 800-999 & 349 & 177 & 0 & 1,716 \\
\hline
\end{tabular}

\footnotetext{
*Absences with >1 ICD-9-CM code in the same diagnostic category were counted only once. Only those diagnostic categories and gender/job category combinations with at least one occurrence appear in this table.
} 
Oak Ridge National Laboratory 2008

Absence Data

Appendix J. Total Number of Calendar Days Absent in Each Diagnostic Category by Gender and Job Category*

\begin{tabular}{|c|c|c|c|c|c|}
\hline & & \multicolumn{4}{|c|}{ Men } \\
\hline & & \multicolumn{4}{|c|}{ Job Category } \\
\hline & & Professional & $\underset{\text { Support }}{\text { Administrative }}$ & Technical Support & Service \\
\hline Diagnostic Category & ICD-9-CM Code & \multirow[b]{2}{*}{127} & \multirow[b]{2}{*}{0} & \multirow[b]{2}{*}{10} & \multirow[b]{2}{*}{16} \\
\hline INFECTIOUS \& PARASITIC DISEASES (DIS) & 001-139 & & & & \\
\hline MALIGNANT NEOPLASMS & $140-208,230-234$ & 248 & 0 & 81 & 41 \\
\hline BENIGN \& UNCERTAIN NEOPLASMS & 210-229, 235-239 & 134 & 0 & 0 & 86 \\
\hline ENDOCRINE/METABOLIC/IMMUNITY & $240-279$ & 121 & 0 & 0 & 2 \\
\hline MENTAL DISORDERS & $290-319$ & 573 & 0 & 10 & 7 \\
\hline NERVOUS SYSTEM (NS) \& SENSE ORGANS & 320-389 & 380 & 0 & 30 & 36 \\
\hline CIRCULATORY SYSTEM & $390-459$ & 281 & 2 & 59 & 174 \\
\hline RESPIRATORY SYSTEM & $460-519$ & 362 & 7 & 106 & 395 \\
\hline DIGESTIVE SYSTEM & 520-579 & 547 & 0 & 92 & 98 \\
\hline GENITOURINARY SYSTEM & 580-629 & 92 & 0 & 4 & 5 \\
\hline SKIN \& SUBCUTANEOUS TISSUE & 680-709 & 97 & 0 & 0 & 34 \\
\hline $\begin{array}{l}\text { MUSCULOSKELETAL \& CONNECTIVE } \\
\text { TISSUE }\end{array}$ & 710-739 & 879 & 0 & 427 & 757 \\
\hline CONGENITAL ANOMALIES & 740-759 & 0 & 0 & 0 & 0 \\
\hline $\begin{array}{l}\text { SYMPTOMS, SIGNS, \& ILL-DEFINED } \\
\text { CONDITIONS }\end{array}$ & 780-799 & 320 & 0 & 53 & 75 \\
\hline INJURY \& POISONING & $800-999$ & 643 & 0 & 708 & 312 \\
\hline
\end{tabular}

\footnotetext{
*Absences with >1 ICD-9-CM code in the same diagnostic category were counted only once. Only those diagnostic categories and gender/job category combinations with at least one occurrence appear in this table.
} 
Oak Ridge National Laboratory 2008

Absence Data

Appendix J. Total Number of Calendar Days Absent in Each Diagnostic Category by Gender and Job Category*

\begin{tabular}{|c|c|c|c|c|c|}
\hline & & \multicolumn{4}{|c|}{ Men } \\
\hline & & \multicolumn{3}{|c|}{ Job Category } & \multirow[b]{2}{*}{ TOTAI } \\
\hline & & Security and Fire & Crafts & Line Operators & \\
\hline Diagnostic Category & ICD-9-CM Code & \multirow[b]{2}{*}{30} & \multirow[b]{2}{*}{288} & \multirow[b]{2}{*}{0} & \multirow[b]{2}{*}{471} \\
\hline INFECTIOUS \& PARASITIC DISEASES (DIS) & $001-139$ & & & & \\
\hline MALIGNANT NEOPLASMS & $140-208,230-234$ & 0 & 51 & 0 & 421 \\
\hline BENIGN \& UNCERTAIN NEOPLASMS & 210-229, 235-239 & 0 & 4 & 1 & 225 \\
\hline ENDOCRINE/METABOLIC/IMMUNITY & $240-279$ & 34 & 0 & 0 & 157 \\
\hline MENTAL DISORDERS & $290-319$ & 0 & 100 & 0 & 690 \\
\hline NERVOUS SYSTEM (NS) \& SENSE ORGANS & $320-389$ & 0 & 161 & 0 & 607 \\
\hline CIRCULATORY SYSTEM & $390-459$ & 0 & 412 & 28 & 956 \\
\hline RESPIRATORY SYSTEM & $460-519$ & 10 & 298 & 10 & 1,188 \\
\hline DIGESTIVE SYSTEM & $520-579$ & 21 & 200 & 43 & 1,001 \\
\hline GENITOURINARY SYSTEM & $580-629$ & 0 & 111 & 0 & 212 \\
\hline SKIN \& SUBCUTANEOUS TISSUE & 680-709 & 0 & 146 & 0 & 277 \\
\hline $\begin{array}{l}\text { MUSCULOSKELETAL \& CONNECTIVE } \\
\text { TISSUE }\end{array}$ & 710-739 & 123 & 2,163 & 0 & 4,349 \\
\hline CONGENITAL ANOMALIES & 740-759 & 0 & 2 & 0 & 2 \\
\hline $\begin{array}{l}\text { SYMPTOMS, SIGNS, \& ILL-DEFINED } \\
\text { CONDITIONS }\end{array}$ & 780-799 & 12 & 101 & 0 & 561 \\
\hline INJURY \& POISONING & $800-999$ & 32 & 2,124 & 83 & 3,902 \\
\hline
\end{tabular}

\footnotetext{
*Absences with >1 ICD-9-CM code in the same diagnostic category were counted only once. Only those diagnostic categories and gender/job category combinations with at least one occurrence appear in this table.
} 
Oak Ridge National Laboratory 2008

Absence Data

Appendix K. Age-Adjusted Illness and Injury Rates by Diagnostic Category*

Part 1. Men

\begin{tabular}{|c|c|c|c|c|c|}
\hline & & $\begin{array}{l}\text { Number of } \\
\text { Diagnoses }\end{array}$ & $\begin{array}{c}\text { Age-Adjusted } \\
\text { Rate per } 1,000 * *\end{array}$ & $\begin{array}{c}\text { Lower } 95 \% \\
\text { Confidence } \\
\text { Limit per } 1,000\end{array}$ & $\begin{array}{c}\text { Upper 95\% } \\
\text { Confidence } \\
\text { Limit per 1,000 }\end{array}$ \\
\hline Diagnostic Category & ICD-9-CM Code & \multirow[b]{2}{*}{34} & \multirow[b]{2}{*}{8.2} & \multirow[b]{2}{*}{5.7} & \multirow[b]{2}{*}{11.9} \\
\hline INFECTIOUS \& PARASITIC DISEASES (DIS) & 001-139 & & & & \\
\hline -Intestinal Infectious Dis & \begin{tabular}{|c|}
$001-009$ \\
\end{tabular} & 1 & 0.1 & 0.0 & 1.0 \\
\hline -Other Bacterial Dis & 030-041 & 11 & 2.3 & 1.2 & 4.4 \\
\hline -Polio \& Other Viral CNS Dis & $045-049$ & 1 & 0.1 & 0.0 & 1.0 \\
\hline -Viral Dis with Exanthem & 050-057 & 2 & 0.4 & 0.1 & 1.7 \\
\hline -Other Viral Dis \& Chlamydiae & \begin{tabular}{|l|} 
070-079 \\
\end{tabular} & 18 & 5.1 & 3.1 & 8.4 \\
\hline -Mycoses & 110-118 & 1 & 0.1 & 0.0 & 1.0 \\
\hline MALIGNANT NEOPLASMS & 140-208, 230-234 & 15 & 3.1 & 1.8 & 5.2 \\
\hline -Digestive \& Peritoneal & $150-159$ & 1 & 0.3 & 0.0 & 1.9 \\
\hline -Genitourinary & 179-189 & 10 & 2.1 & 1.1 & 4.0 \\
\hline -Other \& Unspecified Sites & 190, 193-199 & 3 & 0.4 & 0.1 & 1.3 \\
\hline -Lymphatic \& Hematopoietic & 200-208 & 1 & 0.3 & 0.0 & 1.9 \\
\hline BENIGN \& UNCERTAIN NEOPLASMS & $210-229,235-239$ & 10 & 3.4 & 1.3 & 9.0 \\
\hline ENDOCRINE/METABOLIC/IMMUNITY & $240-279$ & 4 & 1.0 & 0.3 & 2.9 \\
\hline -Other Endocrine Gland Dis & $250-259$ & 1 & 0.1 & 0.0 & 1.0 \\
\hline -Other Metabolic \& Immunity Disorders & 270-279 & 3 & 0.8 & 0.2 & 2.9 \\
\hline MENTAL DISORDERS & $290-319$ & 11 & 2.3 & 1.2 & 4.4 \\
\hline -Psychoses & \begin{tabular}{|l|}
$290-299$ \\
\end{tabular} & 3 & 0.7 & 0.2 & 2.1 \\
\hline -Non-Psychotic Disorders & $300-302,306-316$ & 7 & 1.5 & 0.7 & 3.4 \\
\hline -Alcohol Dependence & 303 & 1 & 0.1 & 0.0 & 1.0 \\
\hline NERVOUS SYSTEM (NS) \& SENSE ORGANS & 320-389 & 36 & 10.6 & 7.0 & 16.2 \\
\hline -Other Disorders of Central NS & $340-349$ & 5 & 2.3 & 0.6 & 8.9 \\
\hline -Disorders of Peripheral NS & $350-359$ & 3 & 0.7 & 0.2 & 2.1 \\
\hline -Disorders of Eye & $360-379$ & 17 & 4.6 & 2.7 & 7.7 \\
\hline -Dis of Ear \& Mastoid & 380-389 & 11 & 3.1 & 1.6 & 5.9 \\
\hline CIRCULATORY SYSTEM & $390-459$ & 45 & 10.9 & 7.4 & 16.1 \\
\hline -Hypertensive Dis & $401-405$ & 7 & 1.4 & 0.7 & 3.1 \\
\hline -Ischemic Heart Dis & $410-414$ & 15 & 3.0 & 1.7 & 5.2 \\
\hline -Dis of Pulmonary Circulation & $415-417$ & 4 & 1.1 & 0.4 & 2.8 \\
\hline -Other Heart Dis & $420-429$ & 7 & 1.6 & 0.7 & 3.7 \\
\hline -Cerebrovascular Dis & $430-438$ & 1 & 0.3 & 0.0 & 1.9 \\
\hline -Dis of Arteries \& Capillaries & $440-448$ & 1 & 0.1 & 0.0 & 1.0 \\
\hline -Dis of Veins, Lymphatics, Other & 451-459 & 10 & 3.4 & 1.3 & 8.9 \\
\hline RESPIRATORY SYSTEM & $460-519$ & 126 & 35.2 & 27.2 & 45.4 \\
\hline -Acute Respiratory Infections & $460-466$ & 38 & 11.1 & 7.0 & 17.6 \\
\hline
\end{tabular}

(Continued)

* Only those diagnostic categories with at least one occurrence appear in this table.

**Standardized to age distribution of 2000 U.S. population. 
Oak Ridge National Laboratory 2008

Absence Data

Appendix K. Age-Adjusted Illness and Injury Rates by Diagnostic Category*

Part 1. Men

\begin{tabular}{|c|c|c|c|c|c|}
\hline & & $\begin{array}{l}\text { Number of } \\
\text { Diagnoses }\end{array}$ & $\begin{array}{c}\text { Age-Adjusted } \\
\text { Rate per } 1,000 * *\end{array}$ & $\begin{array}{c}\text { Lower } 95 \% \\
\text { Confidence } \\
\text { Limit per 1,000 }\end{array}$ & $\begin{array}{c}\text { Upper } 95 \% \\
\text { Confidence } \\
\text { Limit per 1,000 }\end{array}$ \\
\hline Diagnostic Category & ICD-9-CM Code & \multirow[b]{2}{*}{24} & \multirow[b]{2}{*}{7.5} & \multirow[b]{2}{*}{4.0} & \multirow[b]{2}{*}{14.1} \\
\hline -Other Dis Upper Respiratory Tract & $470-478$ & & & & \\
\hline -Pneumonia \& Influenza & $480-487$ & 29 & 8.4 & 5.2 & 13.8 \\
\hline -Chronic Obstructive Dis & $490-496$ & 35 & 8.1 & 5.1 & 13.1 \\
\hline DIGESTIVE SYSTEM & $520-579$ & 88 & 20.4 & 15.4 & 27.2 \\
\hline -Oral Cavity, Saliva Glands, Jaw & $520-529$ & 12 & 5.1 & 2.1 & 12.2 \\
\hline -Esophagus, Stomach, Duodenum & $530-537$ & 6 & 0.9 & 0.4 & 2.2 \\
\hline -Appendicitis & $540-543$ & 2 & 0.3 & 0.1 & 1.1 \\
\hline -Hernia & $550-553$ & 24 & 4.9 & 3.2 & 7.6 \\
\hline -Enteritis, Colitis & $555-558$ & 15 & 3.7 & 2.1 & 6.4 \\
\hline -Other Intestinal Dis & $560-569$ & 12 & 2.3 & 1.2 & 4.3 \\
\hline -Other Digestive Dis & $570-579$ & 17 & 3.2 & 1.9 & 5.3 \\
\hline GENITOURINARY SYSTEM & $580-629$ & 27 & 5.9 & 3.9 & 9.0 \\
\hline -Other Urinary Dis & $590-599$ & 14 & 3.6 & 2.0 & 6.3 \\
\hline -Male Genital Organ Dis & $600-608$ & 13 & 2.4 & 1.3 & 4.2 \\
\hline SKIN \& SUBCUTANEOUS TISSUE & 680-709 & 10 & 4.4 & 1.6 & 11.8 \\
\hline -Infections & $680-686$ & 6 & 3.6 & 1.1 & 11.8 \\
\hline -Other & 700-709 & 4 & 0.8 & 0.3 & 2.2 \\
\hline $\begin{array}{l}\text { MUSCULOSKELETAL \& CONNECTIVE } \\
\text { TISSUE }\end{array}$ & 710-739 & 121 & 28.1 & 22.3 & 35.4 \\
\hline -Arthropathies & $710-719$ & 33 & 8.0 & 4.9 & 13.0 \\
\hline -Dorsopathies & $720-724$ & 52 & 12.0 & 8.4 & 17.3 \\
\hline -Rheumatism, Excluding Back & 725-729 & 29 & 6.5 & 4.3 & 9.7 \\
\hline -Other Dis \& Acquired Deformities & $730-739$ & 7 & 1.6 & 0.7 & 3.6 \\
\hline CONGENITAL ANOMALIES & $740-759$ & 1 & 0.1 & 0.0 & 1.0 \\
\hline $\begin{array}{l}\text { SYMPTOMS, SIGNS, \& ILL-DEFINED } \\
\text { CONDITIONS }\end{array}$ & $780-799$ & 48 & 14.1 & 9.1 & 21.6 \\
\hline -Symptoms & $780-789$ & 38 & 12.3 & 7.6 & 20.0 \\
\hline -Non-Specific Abnormal Findings & $790-796$ & 10 & 1.7 & 0.9 & 3.3 \\
\hline INJURY \& POISONING & 800-999 & 130 & 35.5 & 28.8 & 43.8 \\
\hline -Fracture - Skull & $800-804$ & 4 & 2.2 & 0.5 & 9.0 \\
\hline -Fracture - Upper Limb & 810-819 & 8 & 2.1 & 1.0 & 4.4 \\
\hline -Fracture - Lower Limb & $820-829$ & 3 & 1.9 & 0.4 & 9.4 \\
\hline -Dislocation & 830-839 & 34 & 8.1 & 5.6 & 11.7 \\
\hline -Sprains \& Strains - Back & 846-847 & 10 & 2.2 & 1.1 & 4.2 \\
\hline -Sprains \& Strains - Other & $840-845,848$ & 36 & 9.4 & 6.6 & 13.4 \\
\hline -Internal Injury - Thorax, Abdomen, Pelvis & $860-869$ & 4 & 1.5 & 0.5 & 4.1 \\
\hline
\end{tabular}

(Continued)

* Only those diagnostic categories with at least one occurrence appear in this table.

**Standardized to age distribution of 2000 U.S. population. 
Oak Ridge National Laboratory 2008

Absence Data

Appendix K. Age-Adjusted Illness and Injury Rates by Diagnostic Category*

Part 1. Men

\begin{tabular}{|c|c|c|c|c|c|}
\hline & & $\begin{array}{l}\text { Number of } \\
\text { Diagnoses }\end{array}$ & $\begin{array}{c}\text { Age-Adjusted } \\
\text { Rate per } 1,000 * *\end{array}$ & $\begin{array}{c}\text { Lower } 95 \% \\
\text { Confidence } \\
\text { Limit per 1,000 }\end{array}$ & $\begin{array}{c}\text { Upper 95\% } \\
\text { Confidence } \\
\text { Limit per 1,000 }\end{array}$ \\
\hline Diagnostic Category & ICD-9-CM Code & \multirow[b]{2}{*}{6} & \multirow[b]{2}{*}{1.2} & \multirow[b]{2}{*}{0.5} & \multirow[b]{2}{*}{2.7} \\
\hline -Open Wound - Head, Neck, Trunk & 870-879 & & & & \\
\hline -Superficial Injury & 910-919 & 1 & 0.1 & 0.0 & 1.0 \\
\hline -Contusion & $920-924$ & 11 & 3.1 & 1.7 & 5.7 \\
\hline -Foreign Body Entering Orifice & 930-939 & 1 & 0.4 & 0.1 & 3.1 \\
\hline -Burns & $940-949$ & 2 & 0.7 & 0.2 & 2.9 \\
\hline -Complications \& Unspecified Injuries & \begin{tabular}{|c|}
$958-959$ \\
\end{tabular} & 3 & 1.0 & 0.3 & 3.1 \\
\hline -Unspecified Effects - External Causes & $990-995$ & 1 & 0.3 & 0.0 & 1.9 \\
\hline -Complications of Surgical/Medical Care & 996-999 & 6 & 1.4 & 0.6 & 3.3 \\
\hline Total & & 706 & 183.3 & 165.9 & 202.6 \\
\hline
\end{tabular}

* Only those diagnostic categories with at least one occurrence appear in this table.

**Standardized to age distribution of 2000 U.S. population. 
Oak Ridge National Laboratory 2008

Absence Data

Appendix K. Age-Adjusted Illness and Injury Rates by Diagnostic Category*

Part 2. Women

\begin{tabular}{|c|c|c|c|c|c|}
\hline & & $\begin{array}{l}\text { Number of } \\
\text { Diagnoses }\end{array}$ & $\begin{array}{c}\text { Age-Adjusted } \\
\text { Rate per } 1,000 * *\end{array}$ & $\begin{array}{c}\text { Lower } 95 \% \\
\text { Confidence } \\
\text { Limit per 1,000 }\end{array}$ & $\begin{array}{c}\text { Upper 95\% } \\
\text { Confidence } \\
\text { Limit per 1,000 }\end{array}$ \\
\hline Diagnostic Category & ICD-9-CM Code & \multirow[b]{2}{*}{28} & \multirow[b]{2}{*}{16.3} & \multirow[b]{2}{*}{10.5} & \multirow[b]{2}{*}{25.3} \\
\hline INFECTIOUS \& PARASITIC DISEASES (DIS) & 001-139 & & & & \\
\hline -Intestinal Infectious Dis & 001-009 & 2 & 0.6 & 0.2 & 2.4 \\
\hline -Other Bacterial Dis & 030-041 & 10 & 8.2 & 4.1 & 16.4 \\
\hline -Viral Dis with Exanthem & $050-057$ & 4 & 2.1 & 0.6 & 6.9 \\
\hline -Other Viral Dis \& Chlamydiae & 070-079 & 11 & 5.2 & 2.6 & 10.0 \\
\hline -Mycoses & $110-118$ & 1 & 0.3 & 0.0 & 2.2 \\
\hline MALIGNANT NEOPLASMS & $140-208,230-234$ & 20 & 11.7 & 6.9 & 19.7 \\
\hline -Respiratory \& Intrathoracic & $160-165$ & 1 & 0.3 & 0.0 & 2.2 \\
\hline -Bone, Connective Tissue, Skin & $170-173,176$ & 3 & 0.9 & 0.3 & 2.8 \\
\hline -Breast & 174-175 & 9 & 7.2 & 3.5 & 15.0 \\
\hline -Genitourinary & 179-189 & 1 & 0.3 & 0.0 & 2.2 \\
\hline -Other \& Unspecified Sites & 190, 193-199 & 3 & 2.0 & 0.6 & 7.2 \\
\hline -Carcinoma in situ & $230-234$ & 3 & 0.9 & 0.3 & 2.8 \\
\hline BENIGN \& UNCERTAIN NEOPLASMS & 210-229, 235-239 & 16 & 8.2 & 4.7 & 14.3 \\
\hline ENDOCRINE/METABOLIC/IMMUNITY & 240-279 & 11 & 5.2 & 2.6 & 10.0 \\
\hline -Thyroid Gland Disorders & $240-246$ & 4 & 1.7 & 0.6 & 4.7 \\
\hline -Other Endocrine Gland Dis & $250-259$ & 2 & 0.6 & 0.2 & 2.4 \\
\hline -Other Metabolic \& Immunity Disorders & $270-279$ & 5 & 2.9 & 1.0 & 7.7 \\
\hline BLOOD \& BLOOD-FORMING ORGANS & $280-289$ & 2 & 2.2 & 0.6 & 8.8 \\
\hline MENTAL DISORDERS & 290-319 & 11 & 7.6 & 4.0 & 14.4 \\
\hline -Non-Psychotic Disorders & $300-302,306-316$ & 11 & 7.6 & 4.0 & 14.4 \\
\hline NERVOUS SYSTEM (NS) \& SENSE ORGANS & 320-389 & 40 & 35.6 & 22.3 & 56.9 \\
\hline -Inflammatory Dis of Central NS & $320-326$ & 1 & 3.2 & 0.5 & 22.7 \\
\hline -Hereditary/Degenerative Central NS Dis & 330-337 & 1 & 0.3 & 0.0 & 2.2 \\
\hline -Other Disorders of Central NS & $340-349$ & 7 & 6.6 & 2.3 & 19.0 \\
\hline -Disorders of Peripheral NS & $350-359$ & 17 & 6.0 & 3.5 & 10.4 \\
\hline -Disorders of Eye & $360-379$ & 11 & 14.8 & 6.7 & 32.4 \\
\hline -Dis of Ear \& Mastoid & $380-389$ & 3 & 4.7 & 1.1 & 19.6 \\
\hline CIRCULATORY SYSTEM & $390-459$ & 19 & 12.5 & 7.6 & 20.7 \\
\hline -Hypertensive Dis & $401-405$ & 2 & 0.8 & 0.2 & 3.6 \\
\hline -Ischemic Heart Dis & $410-414$ & 3 & 1.9 & 0.5 & 6.7 \\
\hline -Other Heart Dis & $420-429$ & 1 & 0.5 & 0.1 & 3.8 \\
\hline -Cerebrovascular Dis & $430-438$ & 3 & 2.1 & 0.6 & 7.0 \\
\hline -Dis of Veins, Lymphatics, Other & $451-459$ & 10 & 7.1 & 3.5 & 14.3 \\
\hline RESPIRATORY SYSTEM & $460-519$ & 116 & 88.8 & 68.5 & 115.1 \\
\hline
\end{tabular}

(Continued)

* Only those diagnostic categories with at least one occurrence appear in this table.

**Standardized to age distribution of 2000 U.S. population. 
Oak Ridge National Laboratory 2008

Absence Data

Appendix K. Age-Adjusted Illness and Injury Rates by Diagnostic Category*

Part 2. Women

\begin{tabular}{|c|c|c|c|c|c|}
\hline & & $\begin{array}{l}\text { Number of } \\
\text { Diagnoses }\end{array}$ & $\begin{array}{l}\text { Age-Adjusted } \\
\text { Rate per } 1,000 * *\end{array}$ & $\begin{array}{c}\text { Lower } 95 \% \\
\text { Confidence } \\
\text { Limit per 1,000 }\end{array}$ & $\begin{array}{c}\text { Upper } 95 \% \\
\text { Confidence } \\
\text { Limit per 1,000 }\end{array}$ \\
\hline Diagnostic Category & ICD-9-CM Code & \multirow[b]{2}{*}{25} & \multirow[b]{2}{*}{26.3} & \multirow[b]{2}{*}{15.3} & \multirow[b]{2}{*}{45.0} \\
\hline -Acute Respiratory Infections & $460-466$ & & & & \\
\hline -Other Dis Upper Respiratory Tract & $470-478$ & 30 & 19.2 & 11.8 & 31.4 \\
\hline -Pneumonia \& Influenza & $480-487$ & 26 & 17.8 & 10.0 & 31.6 \\
\hline -Chronic Obstructive Dis & $490-496$ & 32 & 22.1 & 13.4 & 36.4 \\
\hline -Other Respiratory Dis & $510-519$ & 3 & 3.4 & 1.1 & 10.4 \\
\hline DIGESTIVE SYSTEM & \begin{tabular}{|l|}
$520-579$ \\
\end{tabular} & 57 & 49.7 & 34.5 & 71.6 \\
\hline -Oral Cavity, Saliva Glands, Jaw & $520-529$ & 8 & 14.3 & 5.9 & 34.6 \\
\hline -Esophagus, Stomach, Duodenum & $\mathbf{5 3 0 - 5 3 7}$ & 8 & 6.1 & 2.8 & 13.3 \\
\hline -Appendicitis & $540-543$ & 1 & 0.5 & 0.1 & 3.8 \\
\hline -Hernia & $550-553$ & 4 & 3.1 & 1.0 & 9.0 \\
\hline -Enteritis, Colitis & $555-558$ & 13 & 13.1 & 6.1 & 28.2 \\
\hline -Other Intestinal Dis & $560-569$ & 11 & 7.0 & 3.6 & 13.5 \\
\hline -Other Digestive Dis & $570-579$ & 12 & 5.7 & 3.0 & 10.7 \\
\hline GENITOURINARY SYSTEM & $580-629$ & 38 & 28.2 & 17.8 & 44.6 \\
\hline -Nephritis, Nephrosis & $580-589$ & 1 & 0.3 & 0.0 & 2.2 \\
\hline -Other Urinary Dis & $590-599$ & 15 & 7.4 & 4.1 & 13.4 \\
\hline -Breast Disorders & $610-611$ & 1 & 3.2 & 0.5 & 22.7 \\
\hline -Pelvic Inflammatory Dis & 614-616 & 1 & 0.5 & 0.1 & 3.8 \\
\hline -Other Female Disorders & $617-629$ & 20 & 16.8 & 9.0 & 31.1 \\
\hline PREGNANCY \& CHILDBIRTH & $630-677$ & 3 & 5.5 & 1.5 & 19.8 \\
\hline -Pregnancy with Abortive Outcome & $634-639$ & 3 & 5.5 & 1.5 & 19.8 \\
\hline SKIN \& SUBCUTANEOUS TISSUE & 680-709 & 6 & 2.5 & 1.1 & 5.8 \\
\hline -Infections & $680-686$ & 3 & 1.4 & 0.4 & 4.4 \\
\hline -Other Inflammatory Conditions & $690-698$ & 1 & 0.3 & 0.0 & 2.2 \\
\hline -Other & 700-709 & 2 & 0.8 & 0.2 & 3.6 \\
\hline $\begin{array}{l}\text { MUSCULOSKELETAL \& CONNECTIVE } \\
\text { TISSUE }\end{array}$ & 710-739 & 80 & 56.0 & 41.5 & 75.6 \\
\hline -Arthropathies & $710-719$ & 23 & 12.2 & 7.7 & 19.5 \\
\hline -Dorsopathies & $720-724$ & 27 & 29.3 & 17.8 & 48.3 \\
\hline -Rheumatism, Excluding Back & $725-729$ & 24 & 11.4 & 7.2 & 18.1 \\
\hline -Other Dis \& Acquired Deformities & $730-739$ & 6 & 3.0 & 1.2 & 7.5 \\
\hline CONGENITAL ANOMALIES & $740-759$ & 2 & 0.8 & 0.2 & 3.6 \\
\hline $\begin{array}{l}\text { SYMPTOMS, SIGNS, \& ILL-DEFINED } \\
\text { CONDITIONS }\end{array}$ & $780-799$ & 38 & 23.6 & 15.6 & 35.8 \\
\hline -Symptoms & 780-789 & 36 & 22.2 & 14.4 & 34.2 \\
\hline -Non-Specific Abnormal Findings & $790-796$ & 2 & 1.4 & 0.3 & 6.6 \\
\hline
\end{tabular}

(Continued)

* Only those diagnostic categories with at least one occurrence appear in this table.

**Standardized to age distribution of 2000 U.S. population. 
Oak Ridge National Laboratory 2008

Absence Data

Appendix K. Age-Adjusted IIlness and Injury Rates by Diagnostic Category*

Part 2. Women

\begin{tabular}{|c|c|c|c|c|c|}
\hline & & $\begin{array}{l}\text { Number of } \\
\text { Diagnoses }\end{array}$ & $\begin{array}{c}\text { Age-Adjusted } \\
\text { Rate per } 1,000 * *\end{array}$ & $\begin{array}{c}\text { Lower } 95 \% \\
\text { Confidence } \\
\text { Limit per } 1,000\end{array}$ & $\begin{array}{c}\text { Upper } 95 \% \\
\text { Confidence } \\
\text { Limit per 1,000 }\end{array}$ \\
\hline Diagnostic Category & ICD-9-CM Code & \multirow[b]{2}{*}{61} & \multirow[b]{2}{*}{35.1} & \multirow[b]{2}{*}{24.9} & \multirow[b]{2}{*}{49.5} \\
\hline INJURY \& POISONING & $800-999$ & & & & \\
\hline -Fracture - Skull & $\begin{array}{l}800-804 \\
\end{array}$ & 4 & 1.5 & 0.5 & 4.0 \\
\hline -Fracture - Upper Limb & 810-819 & 1 & 0.5 & 0.1 & 3.8 \\
\hline -Fracture - Lower Limb & $820-829$ & 5 & 2.2 & 0.9 & 5.5 \\
\hline -Dislocation & $830-839$ & 12 & 4.9 & 2.6 & 9.2 \\
\hline -Sprains \& Strains - Back & 846-847 & 3 & 6.7 & 1.8 & 25.3 \\
\hline -Sprains \& Strains - Other & $840-845,848$ & 10 & 5.1 & 2.6 & 10.1 \\
\hline -Intracranial Injury & $850-854$ & 1 & 0.5 & 0.1 & 3.8 \\
\hline -Internal Injury - Thorax, Abdomen, Pelvis & $860-869$ & 1 & 0.3 & 0.0 & 2.2 \\
\hline -Open Wound - Head, Neck, Trunk & 870-879 & 6 & 2.5 & 1.1 & 5.8 \\
\hline -Late Effects of Accident & $905-909$ & 1 & 0.3 & 0.0 & 2.2 \\
\hline -Superficial Injury & $910-919$ & 1 & 0.5 & 0.1 & 3.8 \\
\hline -Contusion & $920-924$ & 7 & 3.6 & 1.7 & 7.5 \\
\hline -Complications \& Unspecified Injuries & 958-959 & 1 & 1.2 & 0.2 & 8.2 \\
\hline -Poisoning - Medicinal/Biological & $960-979$ & 1 & 0.5 & 0.1 & 3.8 \\
\hline -Complications of Surgical/Medical Care & 996-999 & 7 & 4.7 & 2.0 & 11.0 \\
\hline Total & & 548 & 389.6 & 346.6 & 438.0 \\
\hline
\end{tabular}

* Only those diagnostic categories with at least one occurrence appear in this table.

**Standardized to age distribution of 2000 U.S. population. 
Oak Ridge National Laboratory 2008

Absence Data

Appendix K. Age-Adjusted Illness and Injury Rates by Diagnostic Category*

Part 3. Men and Women

\begin{tabular}{|c|c|c|c|c|c|}
\hline & & $\begin{array}{l}\text { Number of } \\
\text { Diagnoses }\end{array}$ & $\begin{array}{c}\text { Age-Adjusted } \\
\text { Rate per } 1,000 * *\end{array}$ & $\begin{array}{c}\text { Lower } 95 \% \\
\text { Confidence } \\
\text { Limit per 1,000 }\end{array}$ & $\begin{array}{c}\text { Upper } 95 \% \\
\text { Confidence } \\
\text { Limit per 1,000 }\end{array}$ \\
\hline Diagnostic Category & ICD-9-CM Code & & & & \\
\hline INFECTIOUS \& PARASITIC DISEASES (DIS) & 001-139 & 62 & 10.7 & 8.1 & 14.2 \\
\hline -Intestinal Infectious Dis & 001-009 & 3 & 0.3 & 0.1 & 0.9 \\
\hline -Other Bacterial Dis & 030-041 & 21 & 4.0 & 2.4 & 6.4 \\
\hline -Polio \& Other Viral CNS Dis & 045-049 & 1 & 0.1 & 0.0 & 0.7 \\
\hline -Viral Dis with Exanthem & $050-057$ & 6 & 0.9 & 0.3 & 2.2 \\
\hline -Other Viral Dis \& Chlamydiae & 070-079 & 29 & 5.3 & 3.6 & 7.9 \\
\hline -Mycoses & 110-118 & 2 & 0.2 & 0.0 & 0.8 \\
\hline MALIGNANT NEOPLASMS & $140-208,230-234$ & 35 & 5.7 & 3.9 & 8.2 \\
\hline -Digestive \& Peritoneal & $150-159$ & 1 & 0.2 & 0.0 & 1.6 \\
\hline -Respiratory \& Intrathoracic & $160-165$ & 1 & 0.1 & 0.0 & 0.7 \\
\hline -Bone, Connective Tissue, Skin & 170-173, 176 & 3 & 0.3 & 0.1 & 0.9 \\
\hline -Breast & 174-175 & 9 & 2.0 & 1.0 & 4.2 \\
\hline -Genitourinary & 179-189 & 11 & 1.7 & 0.9 & 3.1 \\
\hline -Other \& Unspecified Sites & $190,193-199$ & 6 & 0.9 & 0.3 & 2.2 \\
\hline -Lymphatic \& Hematopoietic & 200-208 & 1 & 0.2 & 0.0 & 1.6 \\
\hline -Carcinoma in situ & $230-234$ & 3 & 0.3 & 0.1 & 0.9 \\
\hline BENIGN \& UNCERTAIN NEOPLASMS & 210-229, 235-239 & 26 & 4.9 & 2.9 & 8.4 \\
\hline ENDOCRINE/METABOLIC/IMMUNITY & $240-279$ & 15 & 2.3 & 1.3 & 4.1 \\
\hline -Thyroid Gland Disorders & $240-246$ & 4 & 0.5 & 0.2 & 1.5 \\
\hline -Other Endocrine Gland Dis & 250-259 & 3 & 0.3 & 0.1 & 0.9 \\
\hline -Other Metabolic \& Immunity Disorders & 270-279 & 8 & 1.5 & 0.7 & 3.2 \\
\hline BLOOD \& BLOOD-FORMING ORGANS & $280-289$ & 2 & 0.5 & 0.1 & 2.2 \\
\hline MENTAL DISORDERS & 290-319 & 22 & 3.9 & 2.5 & 6.1 \\
\hline -Psychoses & 290-299 & 3 & 0.5 & 0.1 & 1.6 \\
\hline -Non-Psychotic Disorders & $300-302,306-316$ & 18 & 3.3 & 2.0 & 5.5 \\
\hline -Alcohol Dependence & 303 & 1 & 0.1 & 0.0 & 0.7 \\
\hline NERVOUS SYSTEM (NS) \& SENSE ORGANS & 320-389 & 76 & 18.4 & 13.2 & 25.7 \\
\hline -Inflammatory Dis of Central NS & $320-326$ & 1 & 1.0 & 0.1 & 7.3 \\
\hline -Hereditary/Degenerative Central NS Dis & 330-337 & 1 & 0.1 & 0.0 & 0.7 \\
\hline -Other Disorders of Central NS & 340-349 & 12 & 3.6 & 1.6 & 8.4 \\
\hline -Disorders of Peripheral NS & 350-359 & 20 & 2.3 & 1.4 & 3.8 \\
\hline -Disorders of Eye & 360-379 & 28 & 7.7 & 4.6 & 13.0 \\
\hline -Dis of Ear \& Mastoid & 380-389 & 14 & 3.7 & 1.8 & 7.4 \\
\hline CIRCULATORY SYSTEM & $390-459$ & 64 & 11.3 & 8.4 & 15.3 \\
\hline -Hypertensive Dis & $401-405$ & 9 & 1.3 & 0.7 & 2.6 \\
\hline
\end{tabular}

(Continued)

* Only those diagnostic categories with at least one occurrence appear in this table.

**Standardized to age distribution of 2000 U.S. population. 
Oak Ridge National Laboratory 2008

Absence Data

Appendix K. Age-Adjusted Illness and Injury Rates by Diagnostic Category*

Part 3. Men and Women

\begin{tabular}{|c|c|c|c|c|c|}
\hline & & $\begin{array}{l}\text { Number of } \\
\text { Diagnoses }\end{array}$ & $\begin{array}{c}\text { Age-Adjusted } \\
\text { Rate per } 1,000 * *\end{array}$ & $\begin{array}{c}\text { Lower } 95 \% \\
\text { Confidence } \\
\text { Limit per } 1,000\end{array}$ & $\begin{array}{c}\text { Upper } 95 \% \\
\text { Confidence } \\
\text { Limit per 1,000 }\end{array}$ \\
\hline Diagnostic Category & ICD-9-CM Code & \multirow[b]{2}{*}{18} & \multirow[b]{2}{*}{2.7} & \multirow[b]{2}{*}{1.6} & \multirow[b]{2}{*}{4.5} \\
\hline -Ischemic Heart Dis & $410-414$ & & & & \\
\hline -Dis of Pulmonary Circulation & $415-417$ & 4 & 0.8 & 0.3 & 2.2 \\
\hline -Other Heart Dis & $420-429$ & 8 & 1.4 & 0.7 & 3.0 \\
\hline -Cerebrovascular Dis & $430-438$ & 4 & 0.8 & 0.3 & 2.1 \\
\hline -Dis of Arteries \& Capillaries & $440-448$ & 1 & 0.1 & 0.0 & 0.7 \\
\hline -Dis of Veins, Lymphatics, Other & 451-459 & 20 & 4.2 & 2.3 & 7.7 \\
\hline RESPIRATORY SYSTEM & 460-519 & 242 & 51.2 & 42.6 & 61.5 \\
\hline -Acute Respiratory Infections & $460-466$ & 63 & 15.7 & 11.0 & 22.5 \\
\hline -Other Dis Upper Respiratory Tract & $470-478$ & 54 & 10.8 & 7.3 & 16.0 \\
\hline -Pneumonia \& Influenza & $480-487$ & 55 & 11.4 & 7.8 & 16.7 \\
\hline -Chronic Obstructive Dis & $490-496$ & 67 & 12.4 & 8.7 & 17.5 \\
\hline -Other Respiratory Dis & $510-519$ & 3 & 0.9 & 0.3 & 2.7 \\
\hline DIGESTIVE SYSTEM & $520-579$ & 145 & 29.4 & 23.3 & 37.2 \\
\hline -Oral Cavity, Saliva Glands, Jaw & $520-529$ & 20 & 8.0 & 4.3 & 15.0 \\
\hline -Esophagus, Stomach, Duodenum & $530-537$ & 14 & 2.4 & 1.3 & 4.4 \\
\hline -Appendicitis & $540-543$ & 3 & 0.4 & 0.1 & 1.2 \\
\hline -Hernia & $550-553$ & 28 & 4.4 & 2.9 & 6.6 \\
\hline -Enteritis, Colitis & $555-558$ & 28 & 6.5 & 3.9 & 11.1 \\
\hline -Other Intestinal Dis & $560-569$ & 23 & 3.6 & 2.3 & 5.7 \\
\hline -Other Digestive Dis & $570-579$ & 29 & 4.1 & 2.7 & 6.0 \\
\hline GENITOURINARY SYSTEM & $580-629$ & 65 & 12.5 & 8.8 & 17.6 \\
\hline -Nephritis, Nephrosis & $580-589$ & 1 & 0.1 & 0.0 & 0.7 \\
\hline -Other Urinary Dis & 590-599 & 29 & 4.6 & 3.1 & 6.9 \\
\hline -Male Genital Organ Dis & $600-608$ & 13 & 1.7 & 0.9 & 3.0 \\
\hline -Breast Disorders & $610-611$ & 1 & 1.0 & 0.1 & 7.3 \\
\hline -Pelvic Inflammatory Dis & 614-616 & 1 & 0.2 & 0.0 & 1.2 \\
\hline -Other Female Disorders & 617-629 & 20 & 4.9 & 2.6 & 9.3 \\
\hline PREGNANCY \& CHILDBIRTH & $630-677$ & 3 & 1.7 & 0.4 & 6.2 \\
\hline -Pregnancy with Abortive Outcome & 634-639 & 3 & 1.7 & 0.4 & 6.2 \\
\hline SKIN \& SUBCUTANEOUS TISSUE & 680-709 & 16 & 3.8 & 1.7 & 8.3 \\
\hline -Infections & $680-686$ & 9 & 2.9 & 1.0 & 7.9 \\
\hline -Other Inflammatory Conditions & 690-698 & 1 & 0.1 & 0.0 & 0.7 \\
\hline -Other & 700-709 & 6 & 0.8 & 0.4 & 1.9 \\
\hline $\begin{array}{l}\text { MUSCULOSKELETAL \& CONNECTIVE } \\
\text { TISSUE }\end{array}$ & $710-739$ & 201 & 36.9 & 30.6 & 44.4 \\
\hline -Arthropathies & 710-719 & 56 & 9.4 & 6.7 & 13.1 \\
\hline
\end{tabular}

(Continued)

* Only those diagnostic categories with at least one occurrence appear in this table.

**Standardized to age distribution of 2000 U.S. population. 
Oak Ridge National Laboratory 2008

Absence Data

Appendix K. Age-Adjusted Illness and Injury Rates by Diagnostic Category*

Part 3. Men and Women

\begin{tabular}{|c|c|c|c|c|c|}
\hline & & $\begin{array}{l}\text { Number of } \\
\text { Diagnoses }\end{array}$ & $\begin{array}{c}\text { Age-Adjusted } \\
\text { Rate per } 1,000 * *\end{array}$ & $\begin{array}{c}\text { Lower } 95 \% \\
\text { Confidence } \\
\text { Limit per } 1,000\end{array}$ & $\begin{array}{c}\text { Upper 95\% } \\
\text { Confidence } \\
\text { Limit per 1,000 }\end{array}$ \\
\hline Diagnostic Category & ICD-9-CM Code & \multirow[b]{2}{*}{79} & \multirow[b]{2}{*}{17.3} & \multirow[b]{2}{*}{12.6} & \multirow[b]{2}{*}{23.7} \\
\hline -Dorsopathies & $720-724$ & & & & \\
\hline -Rheumatism, Excluding Back & $725-729$ & 53 & 8.2 & 6.1 & 11.1 \\
\hline -Other Dis \& Acquired Deformities & $730-739$ & 13 & 2.0 & 1.1 & 3.6 \\
\hline CONGENITAL ANOMALIES & $740-759$ & 3 & 0.4 & 0.1 & 1.2 \\
\hline $\begin{array}{l}\text { SYMPTOMS, SIGNS, \& ILL-DEFINED } \\
\text { CONDITIONS }\end{array}$ & $780-799$ & 86 & 16.8 & 12.4 & 22.7 \\
\hline -Symptoms & $780-789$ & 74 & 15.3 & 11.0 & 21.2 \\
\hline -Non-Specific Abnormal Findings & 790-796 & 12 & 1.5 & 0.8 & 2.7 \\
\hline INJURY \& POISONING & $800-999$ & 191 & 35.9 & 30.1 & 42.9 \\
\hline -Fracture - Skull & $800-804$ & 8 & 1.9 & 0.6 & 5.8 \\
\hline -Fracture - Upper Limb & 810-819 & 9 & 1.7 & 0.8 & 3.3 \\
\hline -Fracture - Lower Limb & $820-829$ & 8 & 2.0 & 0.7 & 5.8 \\
\hline -Dislocation & 830-839 & 46 & 7.2 & 5.2 & 9.8 \\
\hline -Sprains \& Strains - Back & 846-847 & 13 & 3.7 & 1.6 & 8.4 \\
\hline -Sprains \& Strains - Other & $840-845,848$ & 46 & 8.3 & 6.1 & 11.4 \\
\hline -Intracranial Injury & $850-854$ & 1 & 0.2 & 0.0 & 1.2 \\
\hline -Internal Injury - Thorax, Abdomen, Pelvis & $860-869$ & 5 & 1.1 & 0.4 & 3.0 \\
\hline -Open Wound - Head, Neck, Trunk & $870-879$ & 12 & 1.7 & 0.9 & 3.0 \\
\hline -Late Effects of Accident & 905-909 & 1 & 0.1 & 0.0 & 0.7 \\
\hline -Superficial Injury & $910-919$ & 2 & 0.3 & 0.1 & 1.1 \\
\hline -Contusion & $920-924$ & 18 & 3.4 & 2.1 & 5.4 \\
\hline -Foreign Body Entering Orifice & $930-939$ & 1 & 0.3 & 0.0 & 2.3 \\
\hline -Burns & $940-949$ & 2 & 0.5 & 0.1 & 2.1 \\
\hline -Complications \& Unspecified Injuries & 958-959 & 4 & 1.0 & 0.4 & 2.8 \\
\hline -Poisoning - Medicinal/Biological & $960-979$ & 1 & 0.2 & 0.0 & 1.2 \\
\hline -Unspecified Effects - External Causes & $990-995$ & 1 & 0.2 & 0.0 & 1.6 \\
\hline -Complications of Surgical/Medical Care & 996-999 & 13 & 2.2 & 1.2 & 4.0 \\
\hline Total & & 1254 & 246.3 & 228.1 & 265.9 \\
\hline
\end{tabular}

* Only those diagnostic categories with at least one occurrence appear in this table.

**Standardized to age distribution of 2000 U.S. population. 
Oak Ridge National Laboratory 2008

OSHA Data

Appendix L. Number of Workers with at Least One OSHA Event by Gender, Age, and Job Category*

\begin{tabular}{|c|c|c|c|c|c|c|c|c|c|c|}
\hline \multirow{3}{*}{ Job Category } & \multicolumn{4}{|c|}{ Women } & \multicolumn{5}{|c|}{ Men } & \multirow[b]{3}{*}{ TOTAL } \\
\hline & \multicolumn{3}{|c|}{ Age Group } & \multirow[b]{2}{*}{ TOTAL } & \multicolumn{4}{|c|}{ Age Group } & \multirow[b]{2}{*}{ TOTAL } & \\
\hline & 30 - 39 & $40-49$ & $50+$ & & $16-29$ & 30 - 39 & $40-49$ & $50+$ & & \\
\hline Professional & 2 & 3 & 2 & 7 & 1 & 0 & 5 & 8 & 14 & 21 \\
\hline Administrative Support & 0 & 1 & 4 & 5 & 0 & 0 & 0 & 0 & 0 & 5 \\
\hline Technical Support & 0 & 0 & 0 & 0 & 0 & 1 & 0 & 1 & 2 & 2 \\
\hline Service & 0 & 0 & 2 & 2 & 0 & 1 & 2 & 0 & 3 & 5 \\
\hline Crafts & 0 & 0 & 1 & 1 & 1 & 1 & 3 & 9 & 14 & 15 \\
\hline TOTAL & 2 & 4 & 9 & 15 & 2 & 3 & 10 & 18 & 33 & 48 \\
\hline
\end{tabular}

*Only those job categories and gender/age combinations with at least one OSHA event appear in this table.

Appendix M. Total Number of Workdays Lost or with Restricted Activity from OSHA Events by Gender and Age

\begin{tabular}{|c|c|c|c|c|c|c|c|c|c|}
\hline \multirow[b]{2}{*}{ Age Group } & \multicolumn{3}{|c|}{ Women } & \multicolumn{3}{|c|}{ Men } & \multicolumn{3}{|c|}{ TOTAL } \\
\hline & $\begin{array}{l}\text { Number of } \\
\text { Events }\end{array}$ & $\begin{array}{c}\text { Days } \\
\text { Restricted }\end{array}$ & Days Lost & $\begin{array}{l}\text { Number of } \\
\text { Events }\end{array}$ & $\begin{array}{c}\text { Days } \\
\text { Restricted }\end{array}$ & Days Lost & $\begin{array}{l}\text { Number of } \\
\text { Events }\end{array}$ & $\begin{array}{c}\text { Days } \\
\text { Restricted }\end{array}$ & Days Lost \\
\hline $16-29$ & 0 & 0 & 0 & 2 & 0 & 0 & 2 & 0 & 0 \\
\hline $30-39$ & 2 & 0 & 0 & 3 & 17 & 7 & 5 & 17 & 7 \\
\hline $40-49$ & 4 & 0 & 0 & 10 & 0 & 12 & 14 & 0 & 12 \\
\hline $50+$ & 9 & 7 & 104 & 18 & 2 & 532 & 27 & 9 & 636 \\
\hline TOTAL & 15 & 7 & 104 & 33 & 19 & 551 & 48 & 26 & 655 \\
\hline
\end{tabular}

Appendix N. Total Number of Workdays Lost or with Restricted Activity from OSHA Events by Gender and Job Category*

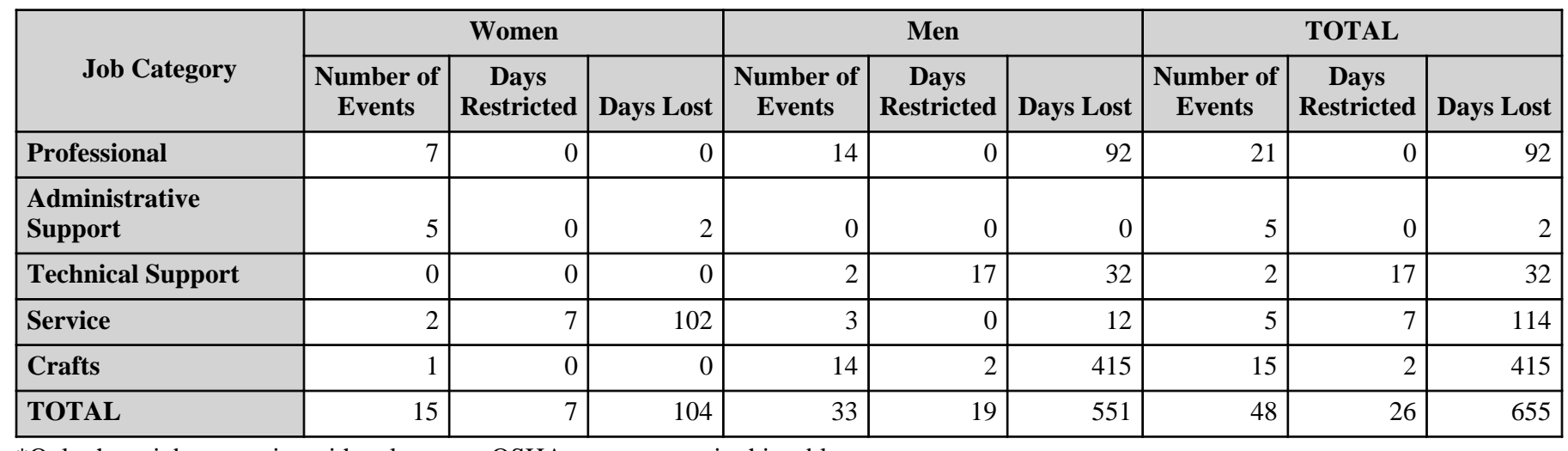

*Only those job categories with at least one OSHA event appear in this table. 
Oak Ridge National Laboratory 2008

OSHA Data

Appendix O. Number of Diagnoses in Each Diagnostic Category by Gender and Age*

\begin{tabular}{|c|c|c|c|c|c|}
\hline & & \multicolumn{4}{|c|}{ Women } \\
\hline & & \multicolumn{3}{|c|}{ Age Group } & \multirow[b]{2}{*}{ TOTAL } \\
\hline & & $30-39$ & $40-49$ & $50+$ & \\
\hline Diagnostic Category & ICD-9-CM code & \multirow[b]{2}{*}{0} & \multirow[b]{2}{*}{0} & \multirow[b]{2}{*}{1} & \multirow[b]{2}{*}{1} \\
\hline RESPIRATORY SYSTEM & $460-519$ & & & & \\
\hline -Other Respiratory Dis & $510-519$ & 0 & 0 & 1 & 1 \\
\hline $\begin{array}{l}\text { MUSCULOSKELETAL \& CONNECTIVE } \\
\text { TISSUE }\end{array}$ & $710-739$ & 0 & 5 & 5 & 10 \\
\hline -Arthropathies & $710-719$ & 0 & 4 & 4 & 8 \\
\hline -Dorsopathies & $720-724$ & 0 & 1 & 1 & 2 \\
\hline -Rheumatism, Excluding Back & 725-729 & 0 & 0 & 0 & 0 \\
\hline $\begin{array}{l}\text { SYMPTOMS, SIGNS, \& ILL-DEFINED } \\
\text { CONDITIONS }\end{array}$ & $780-799$ & 0 & 0 & 4 & 4 \\
\hline -Symptoms & $780-789$ & 0 & 0 & 4 & $\overline{4}$ \\
\hline INJURY \& POISONING & $800-999$ & 2 & 5 & 12 & 19 \\
\hline -Fracture - Upper Limb & $810-819$ & 0 & 0 & 3 & 3 \\
\hline -Fracture - Lower Limb & $820-829$ & 0 & 0 & 1 & 1 \\
\hline -Sprains \& Strains - Back & 846-847 & 0 & 1 & 0 & 1 \\
\hline -Sprains \& Strains - Other & $840-845,848$ & 1 & 1 & 2 & 4 \\
\hline -Open Wound - Head, Neck, Trunk & 870-879 & 0 & 0 & 0 & 0 \\
\hline -Open Wound - Lower Limb & 890-897 & 0 & 0 & 0 & 0 \\
\hline -Superficial Injury & $910-919$ & 0 & 0 & 0 & 0 \\
\hline -Contusion & $920-924$ & 1 & 1 & 3 & 5 \\
\hline -Burns & $940-949$ & 0 & 0 & 0 & 0 \\
\hline -Injury to Nerves \& Spinal Cord & $950-957$ & 0 & 0 & 0 & 0 \\
\hline -Complications \& Unspecified Injuries & 958-959 & 0 & 2 & 2 & 4 \\
\hline -Toxic Effects - Non-medicinal & 980-989 & 0 & 0 & 1 & 1 \\
\hline
\end{tabular}

*Only those diagnostic categories and gender/age combinations with at least one OSHA event appear in this table. 
Oak Ridge National Laboratory 2008

OSHA Data

Appendix O. Number of Diagnoses in Each Diagnostic Category by Gender and Age*

\begin{tabular}{|c|c|c|c|c|c|c|c|}
\hline & & \multicolumn{5}{|c|}{ Men } & \multirow[b]{3}{*}{ TOTAL } \\
\hline & & \multicolumn{4}{|c|}{ Age Group } & \multirow[b]{2}{*}{ TOTAL } & \\
\hline & & $16-29$ & 30 - 39 & $40-49$ & $50+$ & & \\
\hline Diagnostic Category & ICD-9-CM code & & & & & & \\
\hline RESPIRATORY SYSTEM & $460-519$ & 0 & 0 & 0 & 0 & 0 & 1 \\
\hline -Other Respiratory Dis & $510-519$ & 0 & 0 & 0 & 0 & 0 & 1 \\
\hline $\begin{array}{l}\text { MUSCULOSKELETAL \& CONNECTIVE } \\
\text { TISSUE }\end{array}$ & \begin{tabular}{|l|}
$710-739$ \\
\end{tabular} & 0 & 5 & 5 & 15 & 25 & 35 \\
\hline -Arthropathies & 710-719 & 0 & 4 & 4 & 10 & 18 & 26 \\
\hline -Dorsopathies & $720-724$ & 0 & 0 & 0 & 3 & 3 & 5 \\
\hline -Rheumatism, Excluding Back & $725-729$ & 0 & 1 & 1 & 2 & 4 & 4 \\
\hline $\begin{array}{l}\text { SYMPTOMS, SIGNS, \& ILL-DEFINED } \\
\text { CONDITIONS }\end{array}$ & \begin{tabular}{|c|}
$780-799$ \\
\end{tabular} & 0 & 0 & 2 & 2 & 4 & 8 \\
\hline -Symptoms & 780-789 & 0 & 0 & 2 & 2 & 4 & 8 \\
\hline INJURY \& POISONING & 800-999 & 2 & 3 & 11 & 19 & 35 & 54 \\
\hline -Fracture - Upper Limb & 810-819 & 0 & 0 & 1 & 0 & 1 & 4 \\
\hline -Fracture - Lower Limb & 820-829 & 0 & 0 & 0 & 0 & 0 & 1 \\
\hline -Sprains \& Strains - Back & 846-847 & 0 & 0 & 2 & 1 & 3 & 4 \\
\hline -Sprains \& Strains - Other & $840-845,848$ & 0 & 1 & 2 & 6 & 9 & 13 \\
\hline -Open Wound - Head, Neck, Trunk & $870-879$ & 1 & 0 & 1 & 2 & 4 & 4 \\
\hline -Open Wound - Lower Limb & $890-897$ & 0 & 0 & 0 & 1 & 1 & 1 \\
\hline -Superficial Injury & $910-919$ & 0 & 0 & 0 & 1 & 1 & 1 \\
\hline -Contusion & $920-924$ & 0 & 0 & 2 & 2 & 4 & 9 \\
\hline -Burns & 940-949 & 0 & 1 & 0 & 0 & 1 & 1 \\
\hline -Injury to Nerves \& Spinal Cord & $950-957$ & 0 & 0 & 0 & 1 & 1 & 1 \\
\hline -Complications \& Unspecified Injuries & 958-959 & 1 & 1 & 2 & 5 & 9 & 13 \\
\hline -Toxic Effects - Non-medicinal & 980-989 & 0 & 0 & 1 & 0 & 1 & 2 \\
\hline
\end{tabular}

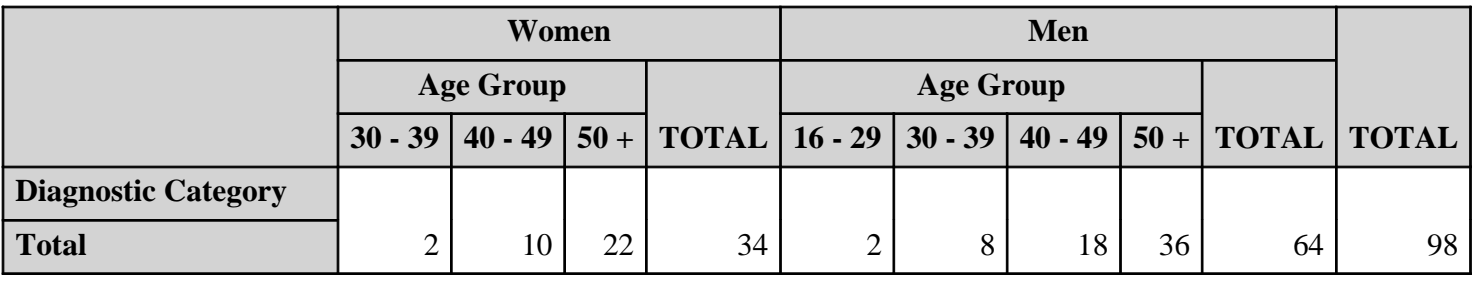

*Only those diagnostic categories and gender/age combinations with at least one OSHA event appear in this table. 
Oak Ridge National Laboratory 2008

OSHA Data

Appendix P. Number of Workdays Lost or with Restricted Activity in Each Diagnostic Category by Gender and Age*

\begin{tabular}{|c|c|c|c|c|c|c|c|}
\hline & & \multicolumn{6}{|c|}{ Women } \\
\hline & & \multicolumn{6}{|c|}{ Age Group } \\
\hline & & \multicolumn{2}{|c|}{30 - 39} & \multicolumn{2}{|c|}{$40-49$} & \multicolumn{2}{|c|}{$50+$} \\
\hline & & $\begin{array}{c}\text { Days } \\
\text { Restricted }\end{array}$ & Days Lost & $\begin{array}{c}\text { Days } \\
\text { Restricted }\end{array}$ & Days Lost & $\begin{array}{c}\text { Days } \\
\text { Restricted }\end{array}$ & Days Lost \\
\hline Diagnostic Category & ICD-9-CM Codes & \multirow[b]{2}{*}{0} & \multirow[b]{2}{*}{0} & \multirow[b]{2}{*}{0} & \multirow[b]{2}{*}{0} & \multirow[b]{2}{*}{0} & \multirow[b]{2}{*}{0} \\
\hline -Other Respiratory Dis & $510-519$ & & & & & & \\
\hline -Arthropathies & $710-719$ & 0 & 0 & 0 & 0 & 0 & 0 \\
\hline -Dorsopathies & $720-724$ & 0 & 0 & 0 & 0 & 0 & 0 \\
\hline -Symptoms & 780-789 & 0 & 0 & 0 & 0 & 0 & 0 \\
\hline -Fracture - Upper Limb & 810-819 & 0 & 0 & 0 & 0 & 0 & 2 \\
\hline -Fracture - Lower Limb & $820-829$ & 0 & 0 & 0 & 0 & 7 & 0 \\
\hline -Sprains \& Strains - Back & $846-847$ & 0 & 0 & 0 & 0 & 0 & 0 \\
\hline -Sprains \& Strains - Other & $840-845,848$ & 0 & 0 & 0 & 0 & 0 & 102 \\
\hline -Contusion & $920-924$ & 0 & 0 & 0 & 0 & 0 & 102 \\
\hline $\begin{array}{l}\text {-Complications \& Unspecified } \\
\text { Injuries }\end{array}$ & 958-959 & 0 & 0 & 0 & 0 & 0 & 2 \\
\hline -Toxic Effects - Non-medicinal & 980-989 & 0 & 0 & 0 & 0 & 0 & 0 \\
\hline
\end{tabular}

\begin{tabular}{|c|c|c|c|c|c|c|c|c|c|}
\hline & \multicolumn{8}{|c|}{ Men } \\
\hline & & \multicolumn{8}{|c|}{ Age Group } \\
\hline & & \multicolumn{2}{|c|}{$16-29$} & \multicolumn{2}{|c|}{30 - 39} & \multicolumn{2}{|c|}{$40-49$} & \multicolumn{2}{|c|}{$50+$} \\
\hline & & $\begin{array}{c}\text { Days } \\
\text { Restricted }\end{array}$ & $\begin{array}{l}\text { Days } \\
\text { Lost }\end{array}$ & $\begin{array}{c}\text { Days } \\
\text { Restricted }\end{array}$ & $\begin{array}{l}\text { Days } \\
\text { Lost }\end{array}$ & $\begin{array}{c}\text { Days } \\
\text { Restricted }\end{array}$ & $\begin{array}{l}\text { Days } \\
\text { Lost }\end{array}$ & $\begin{array}{c}\text { Days } \\
\text { Restricted }\end{array}$ & $\begin{array}{l}\text { Days } \\
\text { Lost }\end{array}$ \\
\hline Diagnostic Category & ICD-9-CM Codes & & & & & & & & \\
\hline -Arthropathies & $710-719$ & 0 & 0 & 0 & 0 & 0 & 0 & 0 & 287 \\
\hline -Dorsopathies & $720-724$ & 0 & 0 & 0 & 0 & 0 & 0 & 2 & 178 \\
\hline -Rheumatism, Excluding Back & 725-729 & 0 & 0 & 0 & 0 & 0 & 0 & 0 & 82 \\
\hline -Symptoms & $780-789$ & 0 & 0 & 0 & 0 & 0 & 0 & 0 & 0 \\
\hline -Fracture - Upper Limb & $810-819$ & 0 & 0 & 0 & 0 & 0 & 0 & 0 & 0 \\
\hline -Sprains \& Strains - Back & 846-847 & 0 & 0 & 0 & 0 & 0 & 0 & 0 & 0 \\
\hline -Sprains \& Strains - Other & $840-845,848$ & 0 & 0 & 0 & 0 & 0 & 0 & 0 & 75 \\
\hline -Open Wound - Head, Neck, Trunk & $870-879$ & 0 & 0 & 0 & 0 & 0 & 0 & 0 & 0 \\
\hline -Open Wound - Lower Limb & $890-897$ & 0 & 0 & 0 & 0 & 0 & 0 & 0 & 0 \\
\hline -Superficial Injury & $910-919$ & 0 & 0 & 0 & 0 & 0 & 0 & 0 & 0 \\
\hline -Contusion & $920-924$ & 0 & 0 & 0 & 0 & 0 & 12 & 0 & 42 \\
\hline -Burns & $940-949$ & 0 & 0 & 17 & 7 & 0 & 0 & 0 & 0 \\
\hline -Injury to Nerves \& Spinal Cord & $950-957$ & 0 & 0 & 0 & 0 & 0 & 0 & 2 & 178 \\
\hline $\begin{array}{l}\text {-Complications \& Unspecified } \\
\text { Injuries }\end{array}$ & 958-959 & 0 & 0 & 0 & 0 & 0 & 0 & 0 & 180 \\
\hline -Toxic Effects - Non-medicinal & $980-989$ & 0 & 0 & 0 & 0 & 0 & 0 & 0 & 0 \\
\hline
\end{tabular}

*OSHA events with >1 ICD-9-CM code in the same diagnostic category were counted only once. Only those diagnostic categories and gender/age combinations with at least one occurrence appear in this table. 
Oak Ridge National Laboratory 2008

OSHA Data

Appendix Q. Number of Occurrences in Each Accident Category by Gender and Age*

\begin{tabular}{|c|c|c|c|c|c|c|c|c|c|c|c|}
\hline & & \multicolumn{4}{|c|}{ Women } & \multicolumn{5}{|c|}{ Men } & \multirow[b]{3}{*}{ TOTAL } \\
\hline & & \multicolumn{3}{|c|}{ Age Group } & \multirow[b]{2}{*}{ TOTAL } & \multicolumn{4}{|c|}{ Age Group } & \multirow[b]{2}{*}{ TOTAL } & \\
\hline & & 30 - 39 & $40-49$ & $\begin{array}{c}50 \\
+\end{array}$ & & $16-29$ & 30 - 39 & $40-49$ & $\begin{array}{c}50 \\
+\end{array}$ & & \\
\hline Type of Accident & E Codes & & & & & & & & & & \\
\hline Motor Vehicle Traffic & E810-E819 & 0 & 0 & 0 & 0 & 0 & 0 & 1 & 2 & 3 & 3 \\
\hline Accidental Poisoning - Non-medicinal & E860-E869 & 0 & 0 & 1 & 1 & 0 & 0 & 0 & 0 & 0 & 1 \\
\hline Falls & E880-E888 & 1 & 2 & 6 & 9 & 0 & 0 & 1 & 3 & 4 & 13 \\
\hline Natural/Environmental Factors & E900-E909 & 0 & 0 & 0 & 0 & 0 & 0 & 1 & 0 & 1 & 1 \\
\hline Other Accidents & E916-E928 & 1 & 2 & 2 & 5 & 2 & 3 & 7 & 13 & 25 & 30 \\
\hline
\end{tabular}

*Only those accident types and gender/age combinations with at least one occurrence appear in this table. 
Oak Ridge National Laboratory 2008

OSHA Data

Appendix R. Number of Workdays Lost or with Restricted Activity in Each Accident Category by Gender and Age*

\begin{tabular}{|c|c|c|c|c|c|c|c|}
\hline & & \multicolumn{6}{|c|}{ Women } \\
\hline & & \multicolumn{6}{|c|}{ Age Group } \\
\hline & & \multicolumn{2}{|c|}{30 - 39} & \multicolumn{2}{|c|}{$40-49$} & \multicolumn{2}{|c|}{$50+$} \\
\hline & & $\begin{array}{c}\text { Days } \\
\text { Restricted }\end{array}$ & Days Lost & $\begin{array}{c}\text { Days } \\
\text { Restricted }\end{array}$ & Days Lost & $\begin{array}{c}\text { Days } \\
\text { Restricted }\end{array}$ & Days Lost \\
\hline Type of Accident & E Codes & \multirow[b]{2}{*}{0} & \multirow[b]{2}{*}{0} & \multirow[b]{2}{*}{0} & \multirow[b]{2}{*}{0} & \multirow[b]{2}{*}{0} & \multirow[b]{2}{*}{0} \\
\hline Accidental Poisoning - Non-medicinal & E860-E869 & & & & & & \\
\hline Falls & E880-E888 & 0 & 0 & 0 & 0 & 7 & 2 \\
\hline Other Accidents & E916-E928 & 0 & 0 & 0 & 0 & 0 & 102 \\
\hline
\end{tabular}

\begin{tabular}{|c|c|c|c|c|c|c|c|c|c|}
\hline & & \multicolumn{8}{|c|}{ Men } \\
\hline & & \multicolumn{8}{|c|}{ Age Group } \\
\hline & & \multicolumn{2}{|c|}{$16-29$} & \multicolumn{2}{|c|}{$30-39$} & \multicolumn{2}{|c|}{$40-49$} & \multicolumn{2}{|c|}{$50+$} \\
\hline & & $\begin{array}{c}\text { Days } \\
\text { Restricted }\end{array}$ & $\begin{array}{l}\text { Days } \\
\text { Lost }\end{array}$ & $\begin{array}{c}\text { Days } \\
\text { Restricted }\end{array}$ & $\begin{array}{l}\text { Days } \\
\text { Lost }\end{array}$ & $\begin{array}{c}\text { Days } \\
\text { Restricted }\end{array}$ & $\begin{array}{l}\text { Days } \\
\text { Lost }\end{array}$ & $\begin{array}{c}\text { Days } \\
\text { Restricted }\end{array}$ & $\begin{array}{l}\text { Days } \\
\text { Lost }\end{array}$ \\
\hline Type of Accident & E Codes & & & & & & & & \\
\hline Motor Vehicle Traffic & E810-E819 & 0 & 0 & 0 & 0 & 0 & 0 & 0 & 0 \\
\hline Falls & E880-E888 & 0 & 0 & 0 & 0 & 0 & 0 & 0 & 42 \\
\hline Natural/Environmental Factors & E900-E909 & 0 & 0 & 0 & 0 & 0 & 0 & 0 & 0 \\
\hline Other Accidents & E916-E928 & 0 & 0 & 17 & 7 & 0 & 12 & 2 & 490 \\
\hline
\end{tabular}

*OSHA events with $>1 \mathrm{E}$ code in the same accident type were counted only once. Only those accident types and gender/age combinations with at least one occurrence appear in this table. 
Oak Ridge National Laboratory 2008

OSHA Data

Appendix S. Number of Diagnoses in Each Diagnostic Category by Gender and Job Category*

\begin{tabular}{|c|c|c|c|c|c|c|}
\hline & & \multicolumn{5}{|c|}{ Women } \\
\hline & & \multicolumn{4}{|c|}{ Job Category } & \multirow[b]{2}{*}{ TOTAL } \\
\hline & & Professional & $\begin{array}{c}\text { Administrative } \\
\text { Support }\end{array}$ & Service & Crafts & \\
\hline Diagnostic Category & ICD-9-CM Code & & & & & \\
\hline $\begin{array}{l}\text { RESPIRATORY } \\
\text { SYSTEM }\end{array}$ & $460-519$ & 0 & 1 & 0 & 0 & 1 \\
\hline -Other Respiratory Dis & $510-519$ & 0 & 1 & 0 & 0 & 1 \\
\hline $\begin{array}{l}\text { MUSCULOSKELETAL } \\
\text { \& CONNECTIVE } \\
\text { TISSUE }\end{array}$ & $710-739$ & 8 & 2 & 0 & 0 & 10 \\
\hline -Arthropathies & 710-719 & 6 & 2 & 0 & 0 & 8 \\
\hline -Dorsopathies & $720-724$ & 2 & 0 & 0 & 0 & 2 \\
\hline $\begin{array}{l}\text { SYMPTOMS, } \\
\text { SIGNS, \& ILL-DEFINED } \\
\text { CONDITIONS }\end{array}$ & $780-799$ & 1 & 3 & 0 & 0 & 4 \\
\hline -Symptoms & $780-789$ & 1 & 3 & 0 & 0 & 4 \\
\hline INJURY \& POISONING & $800-999$ & 8 & 6 & 3 & 2 & 19 \\
\hline -Fracture - Upper Limb & 810-819 & 1 & 2 & 0 & 0 & 3 \\
\hline -Fracture - Lower Limb & $820-829$ & 0 & 0 & 1 & 0 & 1 \\
\hline -Sprains \& Strains - Back & 846-847 & 1 & 0 & 0 & 0 & 1 \\
\hline $\begin{array}{l}\text {-Sprains \& Strains - } \\
\text { Other }\end{array}$ & $840-845,848$ & 3 & 0 & 1 & 0 & 4 \\
\hline -Contusion & $920-924$ & 2 & 1 & 1 & 1 & 5 \\
\hline $\begin{array}{l}\text {-Complications \& } \\
\text { Unspecified Injuries }\end{array}$ & 958-959 & 1 & 2 & 0 & 1 & 4 \\
\hline $\begin{array}{l}\text {-Toxic } \\
\text { Effects - Non-medicinal }\end{array}$ & 980-989 & 0 & 1 & 0 & 0 & 1 \\
\hline
\end{tabular}

\begin{tabular}{|l|r|r|r|r|r|}
\hline \multirow{4}{*}{} & \multicolumn{4}{|c|}{ Women } & \\
\cline { 2 - 5 } & \multicolumn{4}{|c|}{ Job Category } & \multirow{2}{*}{ TOTAL } \\
\cline { 2 - 6 } & Professional & $\begin{array}{c}\text { Administrative } \\
\text { Support }\end{array}$ & Service & Crafts & TOTA \\
\cline { 2 - 6 } Diagnostic Category & & 12 & 3 & 2 & 34 \\
\hline Total & 17 & 12 & & & \\
\hline
\end{tabular}

*Only those diagnostic categories and gender/job category combinations with at least one occurrence appear in this table. 
Oak Ridge National Laboratory 2008

OSHA Data

Appendix S. Number of Diagnoses in Each Diagnostic Category by Gender and Job Category*

\begin{tabular}{|c|c|c|c|c|c|c|}
\hline & \multicolumn{5}{|c|}{ Men } \\
\hline & & \multicolumn{4}{|c|}{ Job Category } & \multirow[b]{2}{*}{ TOTAL } \\
\hline & & Professional & Technical Support & Service & Crafts & \\
\hline Diagnostic Category & ICD-9-CM Code & & & & & \\
\hline $\begin{array}{l}\text { MUSCULOSKELETAL } \\
\text { \& CONNECTIVE } \\
\text { TISSUE }\end{array}$ & 710-739 & 10 & 1 & 2 & 12 & 25 \\
\hline -Arthropathies & $710-719$ & 10 & 0 & 1 & 7 & 18 \\
\hline -Dorsopathies & $720-724$ & 0 & 0 & 0 & 3 & 3 \\
\hline $\begin{array}{l}\text {-Rheumatism, Excluding } \\
\text { Back }\end{array}$ & 725-729 & 0 & 1 & 1 & 2 & 4 \\
\hline $\begin{array}{l}\text { SYMPTOMS, } \\
\text { SIGNS, \& ILL-DEFINED } \\
\text { CONDITIONS }\end{array}$ & 780-799 & 1 & 0 & 1 & 2 & 4 \\
\hline -Symptoms & 780-789 & 1 & 0 & 1 & 2 & 4 \\
\hline INJURY \& POISONING & 800-999 & 17 & 2 & 4 & 12 & 35 \\
\hline -Fracture - Upper Limb & 810-819 & 1 & 0 & 0 & 0 & 1 \\
\hline -Sprains \& Strains - Back & 846-847 & 1 & 0 & 1 & 1 & 3 \\
\hline $\begin{array}{l}\text {-Sprains \& Strains - } \\
\text { Other }\end{array}$ & $840-845,848$ & 4 & 1 & 2 & 2 & 9 \\
\hline $\begin{array}{l}\text {-Open Wound - Head, } \\
\text { Neck, Trunk }\end{array}$ & 870-879 & 1 & 0 & 0 & 3 & 4 \\
\hline $\begin{array}{l}\text {-Open Wound - Lower } \\
\text { Limb }\end{array}$ & 890-897 & 0 & 0 & 0 & 1 & 1 \\
\hline -Superficial Injury & 910-919 & 1 & 0 & 0 & 0 & 1 \\
\hline -Contusion & 920-924 & 3 & 0 & 1 & 0 & 4 \\
\hline -Burns & 940-949 & 0 & 1 & 0 & 0 & 1 \\
\hline $\begin{array}{l}\text {-Injury to } \\
\text { Nerves \& Spinal Cord }\end{array}$ & 950-957 & 0 & 0 & 0 & 1 & 1 \\
\hline $\begin{array}{l}\text {-Complications \& } \\
\text { Unspecified Injuries }\end{array}$ & 958-959 & 6 & 0 & 0 & 3 & 9 \\
\hline $\begin{array}{l}\text {-Toxic } \\
\text { Effects - Non-medicinal }\end{array}$ & 980-989 & 0 & 0 & 0 & 1 & 1 \\
\hline
\end{tabular}

\begin{tabular}{|c|c|c|c|c|c|}
\hline & \multicolumn{5}{|c|}{ Men } \\
\hline & \multicolumn{4}{|c|}{ Job Category } & \multirow[b]{2}{*}{ TOTAL } \\
\hline & Professional & Technical Support & Service & Crafts & \\
\hline Diagnostic Category & \multirow[b]{2}{*}{28} & \multirow[b]{2}{*}{3} & \multirow[b]{2}{*}{7} & \multirow[b]{2}{*}{26} & \multirow[b]{2}{*}{64} \\
\hline Total & & & & & \\
\hline
\end{tabular}

*Only those diagnostic categories and gender/job category combinations with at least one occurrence appear in this table. 
Oak Ridge National Laboratory 2008

OSHA Data

Appendix T. Number of Workdays Lost or with Restricted Activity in Each Diagnostic Category by Gender and Job Category*

\begin{tabular}{|c|c|c|c|c|c|c|c|c|c|}
\hline & & \multicolumn{8}{|c|}{ Women } \\
\hline & & \multicolumn{8}{|c|}{ Job Category } \\
\hline & & \multicolumn{2}{|c|}{ Professional } & \multicolumn{2}{|c|}{$\begin{array}{l}\text { Administrative } \\
\text { Support }\end{array}$} & \multicolumn{2}{|c|}{ Service } & \multicolumn{2}{|c|}{ Crafts } \\
\hline & & $\begin{array}{c}\text { Days } \\
\text { Restricted }\end{array}$ & $\begin{array}{l}\text { Days } \\
\text { Lost }\end{array}$ & $\begin{array}{c}\text { Days } \\
\text { Restricted }\end{array}$ & $\begin{array}{l}\text { Days } \\
\text { Lost }\end{array}$ & $\begin{array}{c}\text { Days } \\
\text { Restricted }\end{array}$ & $\begin{array}{l}\text { Days } \\
\text { Lost }\end{array}$ & $\begin{array}{c}\text { Days } \\
\text { Restricted }\end{array}$ & $\begin{array}{l}\text { Days } \\
\text { Lost }\end{array}$ \\
\hline Diagnostic Category & ICD-9-CM Codes & & & & & & & & \\
\hline -Other Respiratory Dis & $510-519$ & 0 & 0 & 0 & 0 & 0 & 0 & 0 & 0 \\
\hline -Arthropathies & $710-719$ & 0 & 0 & 0 & 0 & 0 & 0 & 0 & 0 \\
\hline -Dorsopathies & $720-724$ & 0 & 0 & 0 & 0 & 0 & 0 & 0 & 0 \\
\hline -Symptoms & $780-789$ & 0 & 0 & 0 & 0 & 0 & 0 & 0 & 0 \\
\hline -Fracture - Upper Limb & 810-819 & 0 & 0 & 0 & 2 & 0 & 0 & 0 & 0 \\
\hline -Fracture - Lower Limb & $820-829$ & 0 & 0 & 0 & 0 & 7 & 0 & 0 & 0 \\
\hline -Sprains \& Strains - Back & \begin{tabular}{|l|}
$846-847$ \\
\end{tabular} & 0 & 0 & 0 & 0 & 0 & 0 & 0 & 0 \\
\hline -Sprains \& Strains - Other & $840-845,848$ & 0 & 0 & 0 & 0 & 0 & 102 & 0 & 0 \\
\hline -Contusion & $920-924$ & 0 & 0 & 0 & 0 & 0 & 102 & 0 & 0 \\
\hline $\begin{array}{l}\text {-Complications \& Unspecified } \\
\text { Injuries }\end{array}$ & 958-959 & 0 & 0 & 0 & 2 & 0 & 0 & 0 & 0 \\
\hline -Toxic Effects - Non-medicinal & $980-989$ & 0 & 0 & 0 & 0 & 0 & 0 & 0 & 0 \\
\hline
\end{tabular}

\begin{tabular}{|c|c|c|c|c|c|c|c|c|c|}
\hline & \multicolumn{8}{|c|}{ Men } \\
\hline & & \multicolumn{8}{|c|}{ Job Category } \\
\hline & & \multicolumn{2}{|c|}{ Professional } & \multicolumn{2}{|c|}{ Technical Support } & \multicolumn{2}{|c|}{ Service } & \multicolumn{2}{|c|}{ Crafts } \\
\hline & & \begin{tabular}{c|} 
Days \\
Restricted
\end{tabular} & $\begin{array}{l}\text { Days } \\
\text { Lost }\end{array}$ & \begin{tabular}{c|} 
Days \\
Restricted
\end{tabular} & $\begin{array}{l}\text { Days } \\
\text { Lost }\end{array}$ & \begin{tabular}{|c|} 
Days \\
Restricted
\end{tabular} & $\begin{array}{l}\text { Days } \\
\text { Lost }\end{array}$ & \begin{tabular}{|c|} 
Days \\
Restricted
\end{tabular} & $\begin{array}{l}\text { Days } \\
\text { Lost }\end{array}$ \\
\hline Diagnostic Category & ICD-9-CM Codes & & & & & & & & \\
\hline -Arthropathies & 710-719 & 0 & 50 & 0 & 0 & 0 & 0 & 0 & 237 \\
\hline -Dorsopathies & 720-724 & 0 & 0 & 0 & 0 & 0 & 0 & 2 & 178 \\
\hline -Rheumatism, Excluding Back & 725-729 & 0 & 0 & 0 & 25 & 0 & 0 & 0 & 57 \\
\hline -Symptoms & $780-789$ & 0 & 0 & 0 & 0 & 0 & 0 & 0 & 0 \\
\hline -Fracture - Upper Limb & $810-819$ & 0 & 0 & 0 & 0 & 0 & 0 & 0 & 0 \\
\hline -Sprains \& Strains - Back & 846-847 & 0 & 0 & 0 & 0 & 0 & 0 & 0 & 0 \\
\hline -Sprains \& Strains - Other & $840-845,848$ & 0 & 50 & 0 & 25 & 0 & 0 & 0 & 0 \\
\hline -Open Wound - Head, Neck, Trunk & 870-879 & 0 & 0 & 0 & 0 & 0 & 0 & 0 & 0 \\
\hline -Open Wound - Lower Limb & $890-897$ & 0 & 0 & 0 & 0 & 0 & 0 & 0 & 0 \\
\hline -Superficial Injury & 910-919 & 0 & 0 & 0 & 0 & 0 & 0 & 0 & 0 \\
\hline -Contusion & $920-924$ & 0 & 42 & 0 & 0 & 0 & 12 & 0 & 0 \\
\hline -Burns & 940-949 & 0 & 0 & 17 & 7 & 0 & 0 & 0 & 0 \\
\hline -Injury to Nerves \& Spinal Cord & 950-957 & 0 & 0 & 0 & 0 & 0 & 0 & 2 & 178 \\
\hline $\begin{array}{l}\text {-Complications \& Unspecified } \\
\text { Injuries }\end{array}$ & 958-959 & 0 & 0 & 0 & 0 & 0 & 0 & 0 & 180 \\
\hline -Toxic Effects - Non-medicinal & 980-989 & 0 & 0 & 0 & 0 & 0 & 0 & 0 & 0 \\
\hline
\end{tabular}

*OSHA events with >1 ICD-9-CM code in the same diagnostic category were counted only once. Only those diagnostic categories and gender/job category combinations with at least one occurrence appear in this table. 
Oak Ridge National Laboratory 2008

OSHA Data

Appendix U. Number of Occurrences in Each Accident Category by Gender and Job Category*

\begin{tabular}{|c|c|c|c|c|c|c|}
\hline & & \multicolumn{5}{|c|}{ Women } \\
\hline & & \multicolumn{4}{|c|}{ Job Category } & \multirow[b]{2}{*}{ TOTAL } \\
\hline & & Professional & $\begin{array}{c}\text { Administrative } \\
\text { Support }\end{array}$ & Service & Crafts & \\
\hline Type of Accident & E CODES & \multirow[b]{2}{*}{0} & \multirow[b]{2}{*}{1} & \multirow[b]{2}{*}{0} & \multirow[b]{2}{*}{0} & \multirow[b]{2}{*}{1} \\
\hline Accidental Poisoning - Non-medicinal & E860-E869 & & & & & \\
\hline Falls & E880-E888 & 5 & 3 & 1 & 0 & 9 \\
\hline Other Accidents & E916-E928 & 2 & 1 & 1 & 1 & 5 \\
\hline
\end{tabular}

\begin{tabular}{|c|c|c|c|c|c|c|}
\hline & & \multicolumn{5}{|c|}{ Men } \\
\hline & & \multicolumn{4}{|c|}{ Job Category } & \multirow[b]{2}{*}{ TOTAL } \\
\hline & & Professional & $\begin{array}{c}\text { Technical } \\
\text { Support }\end{array}$ & Service & Crafts & \\
\hline Type of Accident & E CODES & \multirow[b]{2}{*}{3} & \multirow[b]{2}{*}{0} & \multirow[b]{2}{*}{0} & \multirow[b]{2}{*}{0} & \multirow[b]{2}{*}{3} \\
\hline Motor Vehicle Traffic & E810-E819 & & & & & \\
\hline Falls & E880-E888 & 3 & 0 & 0 & 1 & 4 \\
\hline Natural/Environmental Factors & E900-E909 & 0 & 0 & 0 & 1 & 1 \\
\hline Other Accidents & E916-E928 & 8 & 2 & 3 & 12 & 25 \\
\hline
\end{tabular}

*Only those accident types and gender/job category combinations with at least one occurrence appear in this table. 
Oak Ridge National Laboratory 2008

OSHA Data

Appendix V. Number of Workdays Lost or with Restricted Activity in Each Accident Category by Gender and Job Category*

\begin{tabular}{|c|c|c|c|c|c|c|c|c|c|}
\hline & & \multicolumn{8}{|c|}{ Women } \\
\hline & & \multicolumn{8}{|c|}{ Job Category } \\
\hline & & \multicolumn{2}{|c|}{ Professional } & \multicolumn{2}{|c|}{$\begin{array}{l}\text { Administrative } \\
\text { Support }\end{array}$} & \multicolumn{2}{|c|}{ Service } & \multicolumn{2}{|c|}{ Crafts } \\
\hline & & $\begin{array}{c}\text { Days } \\
\text { Restricted }\end{array}$ & $\begin{array}{l}\text { Days } \\
\text { Lost }\end{array}$ & $\begin{array}{c}\text { Days } \\
\text { Restricted }\end{array}$ & $\begin{array}{l}\text { Days } \\
\text { Lost }\end{array}$ & $\begin{array}{c}\text { Days } \\
\text { Restricted }\end{array}$ & $\begin{array}{l}\text { Days } \\
\text { Lost }\end{array}$ & $\begin{array}{c}\text { Days } \\
\text { Restricted }\end{array}$ & $\begin{array}{l}\text { Days } \\
\text { Lost }\end{array}$ \\
\hline Type of Accident & E Codes & & & & & & & & \\
\hline Accidental Poisoning - Non-medicinal & E860-E869 & 0 & 0 & 0 & 0 & 0 & 0 & 0 & 0 \\
\hline Falls & E880-E888 & 0 & 0 & 0 & 2 & 7 & 0 & 0 & 0 \\
\hline Other Accidents & E916-E928 & 0 & 0 & 0 & 0 & 0 & 102 & 0 & 0 \\
\hline
\end{tabular}

\begin{tabular}{|c|c|c|c|c|c|c|c|c|c|}
\hline & \multicolumn{8}{|c|}{ Men } \\
\hline & & \multicolumn{8}{|c|}{ Job Category } \\
\hline & & \multicolumn{2}{|c|}{ Professional } & \multicolumn{2}{|c|}{ Technical Support } & \multicolumn{2}{|c|}{ Service } & \multicolumn{2}{|c|}{ Crafts } \\
\hline & & \begin{tabular}{|c|} 
Days \\
Restricted
\end{tabular} & $\begin{array}{l}\text { Days } \\
\text { Lost }\end{array}$ & $\begin{array}{c}\text { Days } \\
\text { Restricted }\end{array}$ & $\begin{array}{l}\text { Days } \\
\text { Lost }\end{array}$ & $\begin{array}{c}\text { Days } \\
\text { Restricted }\end{array}$ & $\begin{array}{l}\text { Days } \\
\text { Lost }\end{array}$ & $\begin{array}{c}\text { Days } \\
\text { Restricted }\end{array}$ & $\begin{array}{l}\text { Days } \\
\text { Lost }\end{array}$ \\
\hline Type of Accident & E Codes & & & & & & & & \\
\hline Motor Vehicle Traffic & E810-E819 & 0 & 0 & 0 & 0 & 0 & 0 & 0 & 0 \\
\hline Falls & E880-E888 & 0 & 42 & 0 & 0 & 0 & 0 & 0 & 0 \\
\hline Natural/Environmental Factors & E900-E909 & 0 & 0 & 0 & 0 & 0 & 0 & 0 & 0 \\
\hline Other Accidents & E916-E928 & 0 & 50 & 17 & 32 & 0 & 12 & 2 & 415 \\
\hline
\end{tabular}

*OSHA events with $>1 \mathrm{E}$ code in the same accident type were counted only once. Only those accident types and gender/job category combinations with at least one occurrence appear in this table. 
Oak Ridge National Laboratory 2008

OSHA Data

Appendix W. Age-Adjusted OSHA Illness and Injury Rates by Diagnostic Category*

Part 1. Men

\begin{tabular}{|c|c|c|c|c|c|}
\hline & & $\begin{array}{l}\text { Number of } \\
\text { Diagnoses }\end{array}$ & $\begin{array}{l}\text { Age-Adjusted } \\
\text { Rate per } 1,000 * *\end{array}$ & $\begin{array}{c}\text { Lower } 95 \% \\
\text { Confidence } \\
\text { Limit per } 1,000\end{array}$ & $\begin{array}{c}\text { Upper } 95 \% \\
\text { Confidence } \\
\text { Limit per 1,000 }\end{array}$ \\
\hline Diagnostic Category & ICD-9-CM Code & & & & \\
\hline $\begin{array}{l}\text { MUSCULOSKELETAL \& CONNECTIVE } \\
\text { TISSUE }\end{array}$ & 710-739 & 25 & 5.7 & 3.6 & 8.8 \\
\hline -Arthropathies & $710-719$ & 18 & 4.2 & 2.5 & 7.0 \\
\hline -Dorsopathies & $720-724$ & 3 & 0.5 & 0.2 & 1.8 \\
\hline -Rheumatism, Excluding Back & $725-729$ & 4 & 1.0 & 0.3 & 2.9 \\
\hline $\begin{array}{l}\text { SYMPTOMS, SIGNS, \& ILL-DEFINED } \\
\text { CONDITIONS }\end{array}$ & $780-799$ & 4 & 0.8 & 0.3 & 2.2 \\
\hline -Symptoms & $780-789$ & 4 & 0.8 & 0.3 & 2.2 \\
\hline INJURY \& POISONING & $800-999$ & 35 & 11.0 & 6.9 & 17.5 \\
\hline -Fracture - Upper Limb & 810-819 & 1 & 0.3 & 0.0 & 1.8 \\
\hline -Sprains \& Strains - Back & 846-847 & 3 & 0.8 & 0.3 & 2.4 \\
\hline -Sprains \& Strains - Other & $840-845,848$ & 9 & 2.0 & 1.0 & 4.1 \\
\hline -Open Wound - Head, Neck, Trunk & $870-879$ & 4 & 2.2 & 0.5 & 9.0 \\
\hline -Open Wound - Lower Limb & 890-897 & 1 & 0.1 & 0.0 & 1.0 \\
\hline -Superficial Injury & 910-919 & 1 & 0.1 & 0.0 & 1.0 \\
\hline -Contusion & $920-924$ & 4 & 1.0 & 0.4 & 2.8 \\
\hline -Burns & 940-949 & 1 & 0.4 & 0.1 & 3.1 \\
\hline -Toxic Effects - Non-medicinal & 980-989 & 1 & 0.3 & 0.0 & 1.8 \\
\hline -Complications of Surgical/Medical Care & 996-999 & 10 & 3.7 & 1.5 & 9.2 \\
\hline Total & & 64 & 17.4 & 12.5 & 24.2 \\
\hline
\end{tabular}

* Only those diagnostic categories with at least one occurrence appear in this table.

**Standardized to age distribution of 2000 U.S. population. 
Oak Ridge National Laboratory 2008

OSHA Data

Appendix W. Age-Adjusted OSHA Illness and Injury Rates by Diagnostic Category*

Part 2. Women

\begin{tabular}{|c|c|c|c|c|c|}
\hline & & $\begin{array}{l}\text { Number of } \\
\text { Diagnoses }\end{array}$ & $\begin{array}{c}\text { Age-Adjusted } \\
\text { Rate per } 1,000 * *\end{array}$ & $\begin{array}{c}\text { Lower } 95 \% \\
\text { Confidence } \\
\text { Limit per 1,000 }\end{array}$ & $\begin{array}{c}\text { Upper } 95 \% \\
\text { Confidence } \\
\text { Limit per 1,000 }\end{array}$ \\
\hline Diagnostic Category & ICD-9-CM Code & \multirow[b]{2}{*}{1} & \multirow[b]{2}{*}{1.0} & \multirow[b]{2}{*}{0.1} & \multirow[b]{2}{*}{7.5} \\
\hline RESPIRATORY SYSTEM & $460-519$ & & & & \\
\hline -Other Respiratory Dis & $510-519$ & 1 & 1.0 & 0.1 & 7.5 \\
\hline $\begin{array}{l}\text { MUSCULOSKELETAL \& CONNECTIVE } \\
\text { TISSUE }\end{array}$ & 710-739 & 10 & 4.2 & 2.2 & 8.1 \\
\hline -Arthropathies & $710-719$ & 8 & 3.4 & 1.6 & 7.0 \\
\hline -Dorsopathies & $720-724$ & 2 & 0.8 & 0.2 & 3.6 \\
\hline $\begin{array}{l}\text { SYMPTOMS, SIGNS, \& ILL-DEFINED } \\
\text { CONDITIONS }\end{array}$ & 780-799 & 4 & 3.5 & 1.2 & 9.8 \\
\hline -Symptoms & $780-789$ & 4 & 3.5 & 1.2 & 9.8 \\
\hline INJURY \& POISONING & $800-999$ & 19 & 13.2 & 8.0 & 21.7 \\
\hline -Fracture - Upper Limb & \begin{tabular}{|l|}
$810-819$ \\
\end{tabular} & 3 & 2.4 & 0.7 & 8.3 \\
\hline -Fracture - Lower Limb & 820-829 & 1 & 0.3 & 0.0 & 2.2 \\
\hline -Sprains \& Strains - Back & 846-847 & 1 & 0.5 & 0.1 & 3.8 \\
\hline -Sprains \& Strains - Other & $840-845,848$ & 4 & 3.1 & 1.0 & 9.0 \\
\hline -Contusion & $920-924$ & 5 & 3.4 & 1.2 & 9.1 \\
\hline -Toxic Effects - Non-medicinal & $980-989$ & 1 & 1.0 & 0.1 & 7.5 \\
\hline -Complications of Surgical/Medical Care & 996-999 & 4 & 2.4 & 0.8 & 7.1 \\
\hline Total & & 34 & 21.9 & 15.0 & 31.9 \\
\hline
\end{tabular}

* Only those diagnostic categories with at least one occurrence appear in this table.

**Standardized to age distribution of 2000 U.S. population. 
Oak Ridge National Laboratory 2008

OSHA Data

Appendix W. Age-Adjusted OSHA Illness and Injury Rates by Diagnostic Category*

Part 3. Men and Women

\begin{tabular}{|c|c|c|c|c|c|}
\hline & & $\begin{array}{l}\text { Number of } \\
\text { Diagnoses }\end{array}$ & $\begin{array}{c}\text { Age-Adjusted } \\
\text { Rate per 1,000** }\end{array}$ & $\begin{array}{c}\text { Lower } 95 \% \\
\text { Confidence } \\
\text { Limit per 1,000 }\end{array}$ & $\begin{array}{c}\text { Upper } 95 \% \\
\text { Confidence } \\
\text { Limit per 1,000 }\end{array}$ \\
\hline Diagnostic Category & ICD-9-CM Code & \multirow[b]{2}{*}{1} & \multirow[b]{2}{*}{0.2} & \multirow[b]{2}{*}{0.0} & \multirow[b]{2}{*}{1.6} \\
\hline RESPIRATORY SYSTEM & $460-519$ & & & & \\
\hline -Other Respiratory Dis & $510-519$ & 1 & 0.2 & 0.0 & $\overline{1.6}$ \\
\hline $\begin{array}{l}\text { MUSCULOSKELETAL \& CONNECTIVE } \\
\text { TISSUE }\end{array}$ & \begin{tabular}{|l|l|}
$710-739$ \\
\end{tabular} & 35 & 5.4 & 3.7 & 7.8 \\
\hline -Arthropathies & $710-719$ & 26 & 4.0 & 2.6 & 6.1 \\
\hline -Dorsopathies & $720-724$ & 5 & 0.7 & 0.3 & 1.7 \\
\hline -Rheumatism, Excluding Back & $725-729$ & 4 & 0.7 & 0.2 & 2.1 \\
\hline $\begin{array}{l}\text { SYMPTOMS, SIGNS, \& ILL-DEFINED } \\
\text { CONDITIONS }\end{array}$ & 780-799 & 8 & 1.3 & 0.6 & 2.7 \\
\hline -Symptoms & $780-789$ & 8 & 1.3 & 0.6 & 2.7 \\
\hline INJURY \& POISONING & $800-999$ & 54 & 11.2 & 7.9 & 15.9 \\
\hline -Fracture - Upper Limb & $810-819$ & 4 & 0.7 & 0.3 & 2.0 \\
\hline -Fracture - Lower Limb & $820-829$ & 1 & 0.1 & 0.0 & 0.7 \\
\hline -Sprains \& Strains - Back & 846-847 & 4 & 0.7 & 0.3 & 2.0 \\
\hline -Sprains \& Strains - Other & $840-845,848$ & 13 & 2.3 & 1.3 & 4.1 \\
\hline -Open Wound - Head, Neck, Trunk & $870-879$ & 4 & 1.5 & 0.4 & 6.0 \\
\hline -Open Wound - Lower Limb & $890-897$ & 1 & 0.1 & 0.0 & 0.7 \\
\hline -Superficial Injury & $910-919$ & 1 & 0.1 & 0.0 & 0.7 \\
\hline -Contusion & $920-924$ & 9 & 1.7 & 0.8 & 3.4 \\
\hline -Burns & 940-949 & 1 & 0.3 & 0.0 & 2.3 \\
\hline -Toxic Effects - Non-medicinal & $980-989$ & 2 & 0.4 & 0.1 & 1.6 \\
\hline -Complications of Surgical/Medical Care & 996-999 & 14 & 3.3 & 1.6 & 6.8 \\
\hline Total & & 98 & 18.1 & 14.1 & 23.2 \\
\hline
\end{tabular}

* Only those diagnostic categories with at least one occurrence appear in this table.

**Standardized to age distribution of 2000 U.S. population. 